\title{
Les Hymenolepis (sensu lato) de Charadriiformes
}

\section{(A propos de 23 descriptions)}

\author{
Par Stéphane DEBLOCK et Franeis ROSÉ
}

Plan

A) Introduction.

B) Historique. Liste et discussion de la cinquantaine d'espèces signalées, COMPORTANT UNE REDESCRIPTION COMPLÈTE OU PARTIELLE DES ESPÈCES SUIVANTES :

- Hymenolepis (Hym.) amphitricha (Rud., 1819).

- Hymenolepis (H.) cambrensis Davies, 1939.

- Hymenolepis $(H$.) charadrii Yamaguti, 1935.

- Hymenolepis $(\boldsymbol{H}$.) clandestina (Krabbe, 1869).

- Hymenolepis $(\boldsymbol{H}$.) collariella Coil, 1956.

- Hymenolepis $(H$.$) crocethia (Belop., 1954).$

- Hymenolepis $(H$.$) crocethiae (Webster, 1947).$

- Hymenolepis (H.) guschanskoi (Krotov, 1952).

- Hymenolepis $(\boldsymbol{H}$.) hughesi Webster, 1947.

- Hymenolepis $(\boldsymbol{H}$.) lauriei Davies, 1939.

- Hymenolepis (H.) litoralis (Webster, 1947).

- Hymenolepis (H.) mudderbugtenensis (Baer, 1956).

- Hymenolepis $(H$.$) nitida (Krabbe, 1869).$

- Hymenolepis (Echinocotyle) nitida Clerc, 1902-1903.

- Hymenolepis (Echinocotyle) paradoxus (Saakova, 1958) nov. comb.

C) Description d'ESPèces Nouvelles :

1. Hym. (Hym.) belopolskaiae.

2. Hym. (Hym.) capetownensis.

3. Hym. (Hym.) etaplesensis.

4. Hym. (Hym.) hughesi nov, var. europaei.

5. Hym. (Hym.) patersoni. 
D) Description de species inquirendae (DE LA FAunE dE France).

1. Hymenolepis (Hym.) sp. inq. $\mathrm{n}^{\circ} 1$.

2. Hymenolepis (Echinocotyle?) sp. inq., $\mathrm{n}^{\circ} 2$ (= hypoleuci ?).

3. Hymenolepis (Echinocotyle) sp. inq. $\mathrm{n}^{\circ} 3$ (= glareolae ?).

E) Essai de Clé diagnostique.

F) Conclusion.

\section{A) INTRODUCTION}

L'autopsie de Charadrii capturés le long des côtes de la Manche au cours de plusieurs années nous a permis la récolte d'espèces d'Hymenolepis connues ou non. A ce lot initial devaient s'adjoindre plusieurs préparations d'Hymenolepis demandées en consultation, car la systématique de ce groupe de Cestodes nous semblait établie sur des données incomplètes ou insuffisantes. La description totale ou partielle de ces espèces portant essentiellement sur les conduits génitaux, leur illustration, leur discussion, la révision des espèces du genre feront l'objet du présent mémoire.

Nous remercions très vivement et très sincèrement les helminthologistes qui ont bien voulu nous honorer de leur confiance en nous prêtant un matériel original irremplaçable: M. le Professeur J.-G. Baer, de l'Université de Neuchâtel ; Mmes les Professeurs M. M. Belopolskaia, de la Faculté des Sciences de Léningrad, et F. Rees, de l'University College of Wales; M. A. C. Smith, conservateur du Musée National d'Histoire Naturelle des Etats-Unis d'Amérique ; M. F. Bona, Assistant à 1'Université de Turin.

Nos remerciements vont aussi à notre ami le Professeur A.-G. Chabaud pour le don de ses récoltes du Canet.

La terminologie utilisée dans ce travail et l'emplacement de quelques mensurations particulières sont précisés sur le schéma théorique des terminaisons génitales mâles et femelles, rassemblant toutes les anatomies que nous avons observées chez les Hymenolepis de Charadrii (fig. 1), de même que la figure 2 rappelle la nomenclature de sept types de positions des glandes génitales.

Enfin, nous distinguons trois silhouettes principales de crochets du rostre :

$1^{\circ}$ type I: crochets « chéliformes ; ex.: Hym. (Hym.) amphitricha (Rud., 1819); cf. fig. 4.

$2^{\circ}$ type II : crochets « falciformes $\gg ;$ ex. : Hym. (Hym.) nitida (Krabbe, 1869); cf. fig. 29.

$3^{\circ}$ type III : crochets « claviformes 》 rappelant la silhouette d'une clé à molette ; ex. : Hym. (Hym.) cambrensis Davies, 1939; cf. fig. 9. Le type III groupe en fait toutes les silhouettes qui s'éloignent nettement des deux premières, très caractéristiques. 
N. B. - Toutes les échelles des figures, tracées à la chambre claire sauf exception mentionnée, sont indiquées en microns.

\section{B) HISTORIQUE - REVUE DES ESPECES CONNUES}

On a signalé chez les Charadrii 54 espèces de Cestodes du genre Hymenolepis; nous incluons dans celui-ci deux sous-genres, les seules bien différenciables: $1^{\circ}$ Hymenolepis (Hymenolepis) Weinland, 1858 et $2^{\circ}$ Hymenolepis (Echinocotyle) Blanchard, 1891. Abstraction est faite dans cette liste des subdivisions génériques proposées par López-Neyra (1942) et par Spassky et Spasskaya à partir de 1954. Le démembrement de ce genre surpeuplé est souhaitable. Cependant, les connaissances morphologiques de beaucoup de ces vers, fragmentaires, imprécises ou mal interprétées, handicapent au départ ces tentatives de trop d'imperfection. Très contractés ou, au contraire, convenablement relâchés, ils passent d'un genre à l'autre, par la modification des rapports de position des glandes génitales entre elles. Ces Cestodes, par ailleurs, semblent vouloir défier toute classification rationnelle, par la multiplicité des combinaisons des caractères simples définissant les espèces.

L'énumération des espèces parasites des Charadrii par ordre alphabétique sera suivie pour plusieurs d'entre elles d'une discussion basée sur l'examen critique de la littérature les concernant, ou sur un complément de description et d'iconographie résultant de nos propres observations.

1) HYMENOLEPIS (HYM.) AMPHITRICHA (Rud., 1819)

= Hymenolepis (Drepanidotaenia) $a$. Cohn, 1901 et Clerc, 1903 ; Weinlandia $a$. Mayhew, 1925 ; Dicranotaenia a. López-Neyra, 1942 ; Limnolepis a. Spassky et Spasskaïa, 1954.

Région paléarctique (Europe : Bremser et Krabbe, 1869; Russie d'Europe (Clerc, 1903 ; Cholodkowsky, 1912 ; Belopolskaïa, 1959) et d'Asie (Dubinina, 1953 ; Panin, 1960).

Hôtes: Erolia alpina (L.), Tringa maritima Brünn., Scolopax rusticola L., Tringa ochropus L., Capella gallinago (L.), Limosa limosa (L.).

Espèce signalée également chez Nyroca marila (L.), Ansériforme, au Kamtchatka, par Spassky et Bobova, 1962.

La redescription de Clerc (1903) n'apporte que peu de précisions chiffrées et aucune illustration de cette espèce, caractéristique par la puissance de son cirre, retrouvée en France en 1959 sur le littoral de la Manche (Baie de Canche) chez Erolia (= Tringa) alpina (L.) en plusieurs exemplaires. 


\section{Redescription de l'espèce.}

1) TAille: longueur totale, 4 à $10 \mathrm{~cm} \times 2,5 \mathrm{~mm}$ de largeur maximale. Les anneaux du cou très étroits s'élargissent de façon assez rapide sur certains strobiles longs de $4 \mathrm{~cm}$ ne comportant que 210 anneaux, et de façon beaucoup plus lente sur des strobiles longs de $10 \mathrm{~cm}$ pourvus de 1.000 et 1.500 anneaux.

2) Scolex (fig. 3 et 4): petit, globuleux, muni de quatre ventouses arrondies non spinulées et d'un rostre armé rétractile; diamètre transverse, 150$170 \times 160-180 \mu$ de longueur. Ventouses circulaires de 40 à $60 \mu$ de diamètre ; épaisseur du bord musculaire : 10-15 $\mu$.

Rostre évaginé court, $53 \mu$ de long $\times 50 \mu$ de large à la sortie du scolex ; table apicale de $50 \mu$ de diamètre. Une couronne de 10 crochets chéliformes pratiquement sans manche, à garde longue, bien que plus courte "e la lame sinueuse très effilée ; longs de $22-23 \mu \times 8 \mu$ de hauteur ; base lougue de 11 à $14 \mu$. Sac du rostre : $120 \times 50 \mu$, dépassant légèrement le bord inférieur des ventouses.

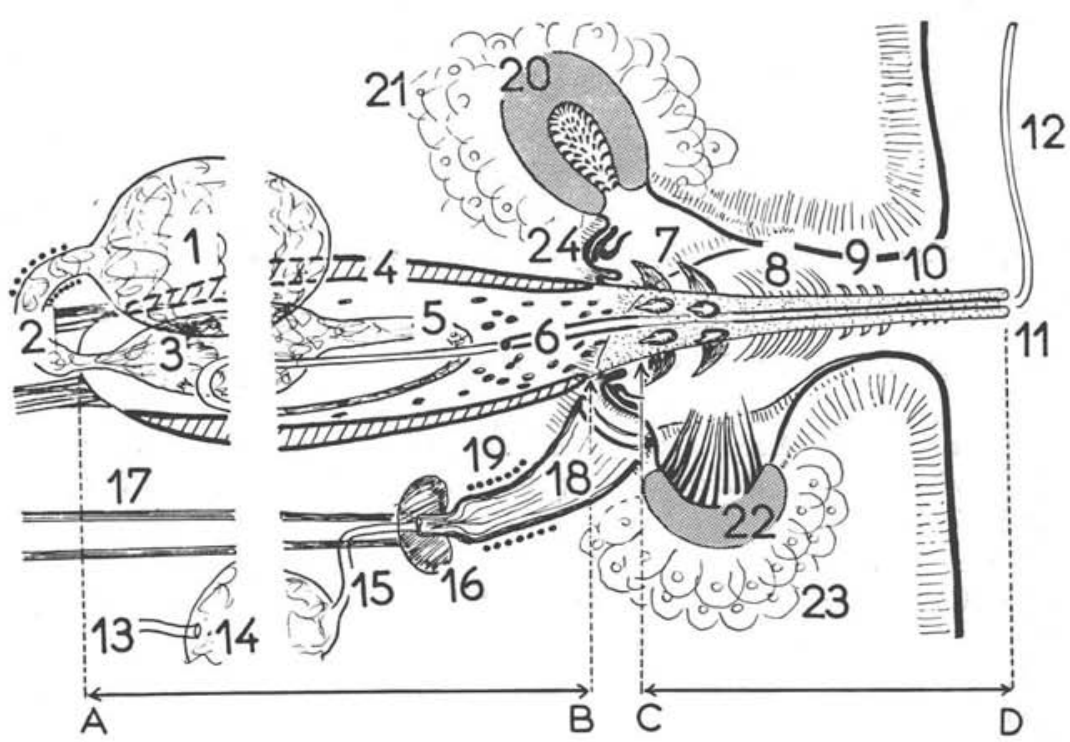

Fig. 1. - Conduits sexuels mâle et femelle et atrium génital (schéma théorique) A) Conduits mâles: 1 . Vésicule séminale externe. - 2. Canal déférent externe. - 3. Vésicule séminale interne. - 4. Poche du cirre. - 5. Canal éjaculateur. - 6. Tube chitineux. - 7 à 10. Ornementations du cirre: fortes épines (7), soies (8), petites épines (9), épines punctiformes (10). - 11. Cirre évaginé. - 12. Stylet. - B) Conduits femelles : 13. Canal séminal. 14. Réceptacle séminal. - 15. Vagin proximal (non copulateur). - 16. Sphincter vaginal. 17. Fibres rétractrices du vagin. - 18. Vagin distal (copulateur). - 19. Fibres annulaires. 20. Sac accessoire. - 21. Glande ciu sac. - 22. Sac accessoire du type «peigne atrial» et ses soies. - 23. Glande du sac. - 24. Epines atriales. - AB : mesure de la poche du cirre. $\mathrm{CD}$ : mesure du cirre. $\mathrm{C}$ : mesure du diamètre basal du cirre. $\mathrm{D}:$ mesure du diamètre distal. 


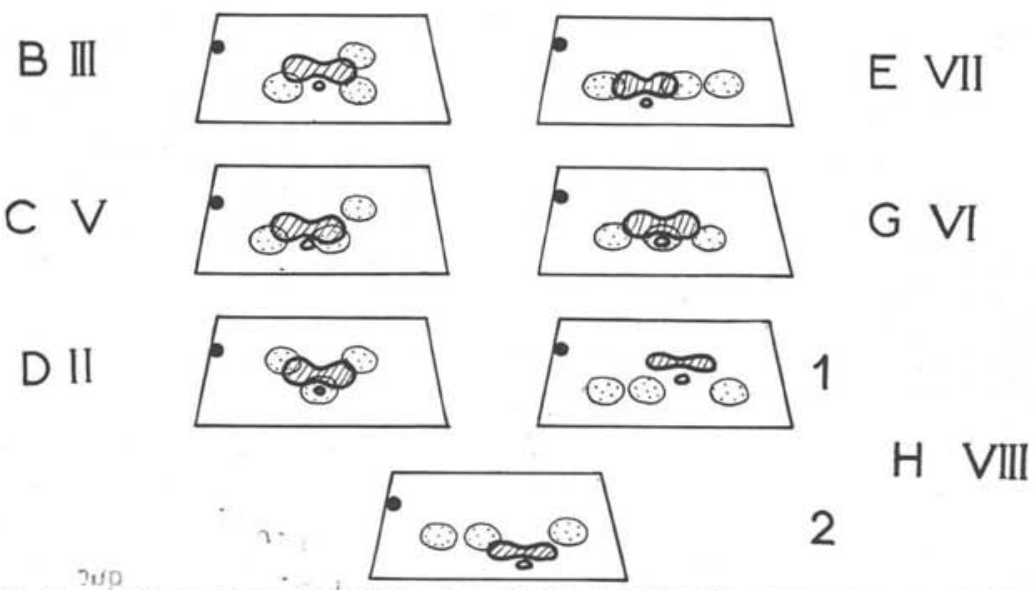

FIg. 2. Divers types de position des glandes génitales (en majuscules, nomenclature selon Fuhrmann, 1932, fig. 111, p. 136; en chiffres romains, nomenclature selon Skriabine et Mathévossian, 1945).

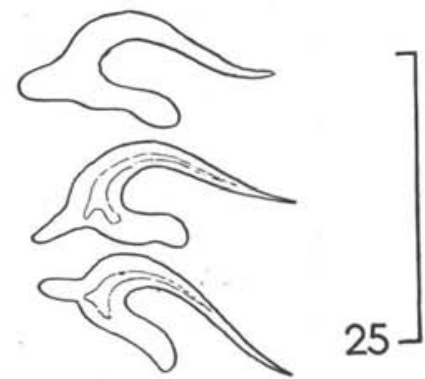

Fig. 4. - H. (H.) amphitricha. Crochets du rostre.

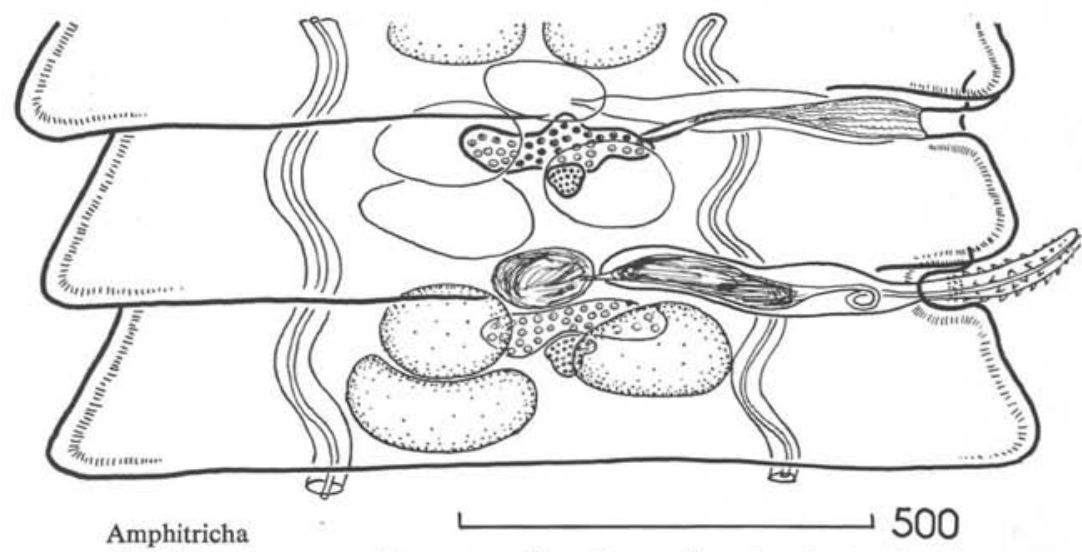

FIG. 5. - Anneaux mâles et massifs emb̧ryonnaires des glandes femelles. Ann. de Parasitologie, T. XXXVII, No 5-6. -1962. 


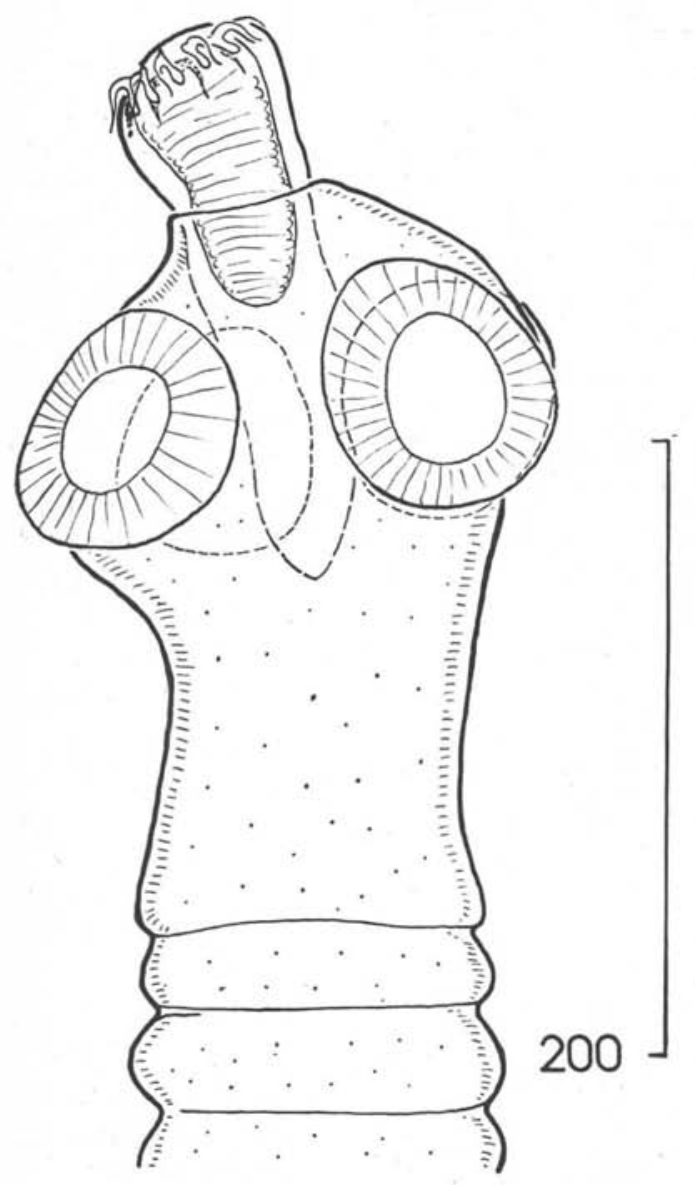

Fig. 3. $-H$. (H.) amphitricha (Rud.).

Erolia alpina (L.). (Baie de Canche, 1959). Scolex.

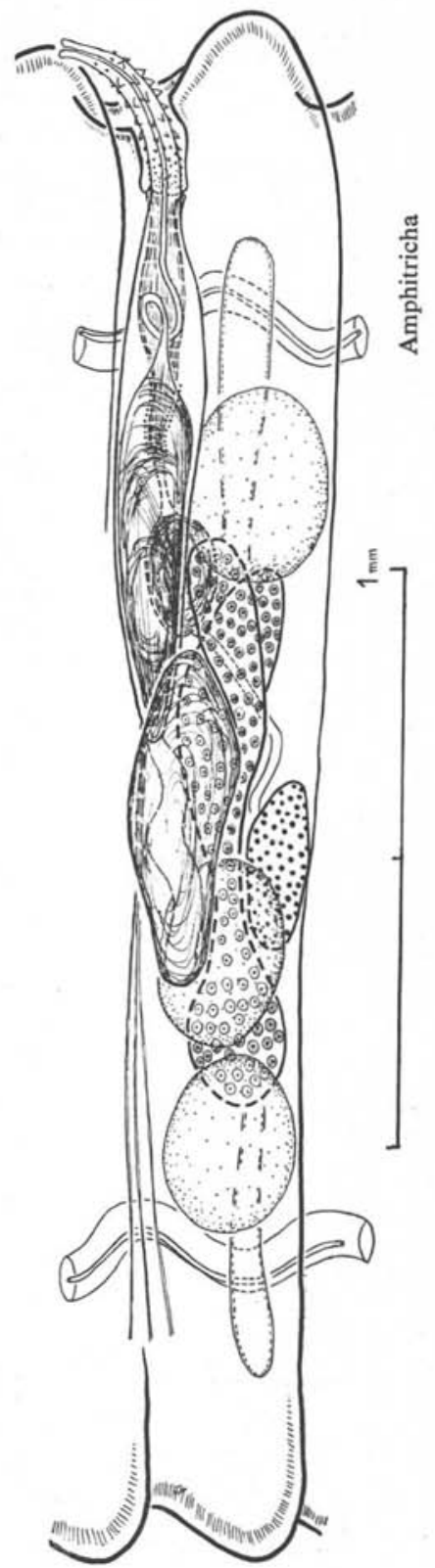

Fig. 6. $-H$. $(H$.$) amphitricha. Anneau mûr. Vue dorsale.$ 
3) Cou : court, large de $120 \mu$. La strobilisation commence très rapidement, à $150 \mu$ en arrière du bord postérieur des ventouses.

4) Strobile: nombre d'anneaux variable selon les exemplaires: 210 anneaux à 1.000-1.500. Toujours de forme trapézoïdale très allongée et peu imbriqués les uns dans les autres. L'exemplaire de $40 \mathrm{~mm}$ compte une cinquantaine d'anneaux immatures, 50 anneaux sexués et 200 anneaux gravides dont la maturation des œufs est très lente.

Musculature interne: formée sur chacune des faces du Cestode de 30-35 fibres longitudinales externes de 4-5 $\mu$ de diamètre et de 12 à 15 fibres internes de $10-12 \mu$ de diamètre.

CANAUX EXCRÉTEURS : une paire de canaux de chaque côté, courant à $100 \mu$ du bord de l'anneau, ventraux par rapport aux conduits sexuels. Diamètre du canal ventral : $20 \mu$-50 $\mu$, et du dorsal : $5,5 \mu$. Il ne semble pas y avoir d'anastomose transverse entre les canaux ventraux.

Atrium géNITAL : dextre, tubulaire, dépourvu de sacculus et d'épine.

\section{a) Anneaux mâles (fig. 5 et 6 )}

Trapézoïdaux. Beaucoup plus larges que longs. Rapport largeur/longueur = 1/5. Taille moyenne : $980-1.200 \mu \times 185-200 \mu$. Protérandrie plus ou moins accusée suivant les exemplaires. Les trois testicules se situent dorsalement, en triangle, préférentiellement suivant le type B ; taille : $130 \times 80 \mu$. Les massifs embryonnaires des glandes femelles naissent ventralement au centre de l'anneau, entre les trois testicules. Dans les anneaux plus âgés, les testicules se placent en ligne, le testicule antérieur venant se glisser en arrière, entre les deux postérieurs.

POCHE DU CIRRE : limitée par une paroi musculaire mince $(2,5 \mu)$ formée de fibres non hélicoïdales, elle dépasse largement les canaux osmo-régulateurs poraux mais n'atteint qu'à peine l'axe longitudinal du strobile.

Taille: 1) dans les anneaux mâles de $1.350 \times 200 \mu$, elle mesure $370 \times$ $50-80 \mu ; 2)$ dans des anneaux mûrs de $1.650 \times 225 \mu$, elle atteint $470 \times 80 \mu$; $\mathrm{PC} / \mathrm{AM}=1 / 3,5(1) ; 3$ ) dans les anneaux gravides de $2.900 \times 260 \mu$, elle croît encore jusqu'à $700 \times 180 \mu$ de diamètre. $\mathrm{PC} / \mathrm{AG}=1 / 4$. Sur le fond de la poche, s'insèrent quelques fibres rétractrices modérément développées.

La vÉSICULE SÉMINALE INTERNE en occupe les $2 / 3$ antiporaux. Le canal éjaculateur décrit toujours une boucle dans le tiers poral de la poche entre son départ du sommet poral de la vésicule séminale et son engagement dans l'axe du cirre ; cette boucle s'efface plus ou moins en fonction de l'état d'évagination de ce dernier jusqu'à devenir tout à fait rectiligne.

(1) Rapport de la longueur de la poche du cirre non contractée (P.C.), à la largeur de l'anneau mûr (A.M.). 


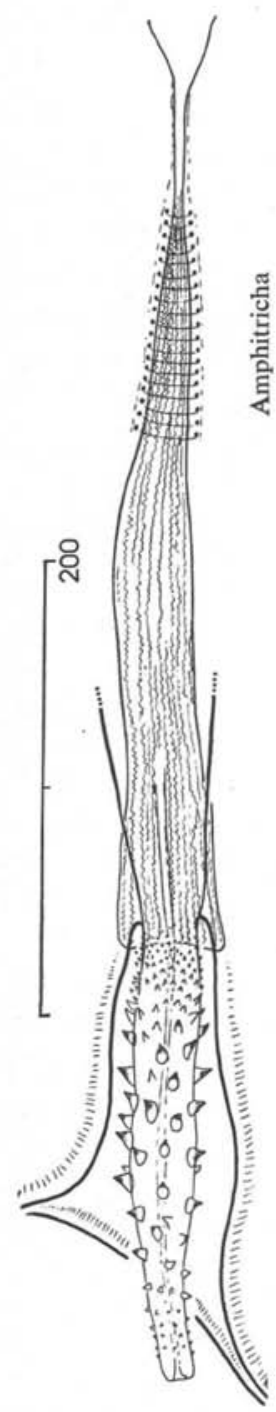

FIG. 7. - H. (H.) amphitricha. Conduits sexuels terminaux et atrium génital. Vue ventrale.

CIrre : 1) A l'état invaginé, les épines sont bien visibles sur une longueur de $130 \mu$ à l'intérieur de la poche.

2) A l'état évaginé, le cirre mesure $210 \mu \times 30 \mu$. Cylindrique à extrémité légèrement conique. Très épineux sur la presque totalité de la longueur: à la base, les épines sont denses et petites $(2 \mu$ de long) sur une longueur de 30-35 $\mu$, très fortes et plus rares $(8 \mu \times 6,5$ large $\times$ 3-4 $\mu$ d'épaisseur à la partie moyenne), diminuant en taille et augmentant en nombre au fur et à mesure qu'on se rapproche de l'extrémité du cirre, qui est complètement glabre sur une cinquantaine de microns et large de $15 \mu$ (fig. 7).

VÉSICUle SÉminale EXTERNE: taille: $190 \times 100 \mu$; se situe dorsalement dans le prolongement de la poche du cirre, sensiblement au niveau de l'axe longitudinal du strobile, juste en avant de l'ovaire. Le canal déférent externe, long de $140 \times 15-40 \mu$ de diamètre, l'aborde par son extrémité antiporale. Il est entouré de fibres musculaires circulaires sur tout son parcours.

\section{b) Anneaux mûrs (fig. 6)}

OvaIre : trilobé, allongé, médian, ventral ; taille : 200$340 \mu \times 50-100 \mu$ à maturité, juste avant la ponte ovulaire.

VITELloGÊNE : ovoïde, médian, postérieur à l'ovaire ; taille : $125 \times 60 \mu$.

RÉCEPTAClE SÉMINAL: ovoïde. Situé ventralement au niveau du lobe poral de l'ovaire. Taille : de $117 \times 37 \mu$ jusqu'à $320 \times 130 \mu$. Il émet au pôle opposé au vagin un long canal séminal $(130 \times 12 \mu$ de diamètre $)$.

VAGIN : droit, long de $220 \times 25 \mu$ de diamètre maximal, de forme cylindro-conique très effilée. Sa portion distale copulatrice constitue à elle seule les $4 / 5^{\circ}$ de la longueur totale ; sa paroi chitinoïde est très mince et parcourue de très nombreux petits plis longitudinaux finement onduleux ; entourée de fibres musculaires annulaires bien visibles sur une longueur d'une soixantaine de microns au niveau de son rétrécissement. Ni sphincter, ni fibres rétractrices.

\section{c) Anneaux gravides}

Constituent les anneaux les plus nombreux du strobile. Taille moyenne: 2.000-2.500 $\times 250-350 \mu$. Poche du cirre, vésicule séminale externe, vagin et réceptacle séminal y persistent. 
UTÉRUS: Sacculaire multilobé, dépassant les canaux osmo-régulateurs; contient un grand nombre d'œufs de forme sphérique ou légèrement ovoïde. Eurs : les plus mûrs en notre possession n'ont pas encore atteint leur complète maturation. L'embryon hexacanthe mesure de $25 \mu$ de diamètre à $25 \times$ $31 \mu$; la coque externe de $45 \times$ $57 \mu$ à $58 \times 75 \mu$ est uniformément mince et incolore. La coque interne n'est pas visible. Les crochets sont hétérogènes, les crochets internes des paires latérales étant plus développés que les quatre autres (fig. 8).

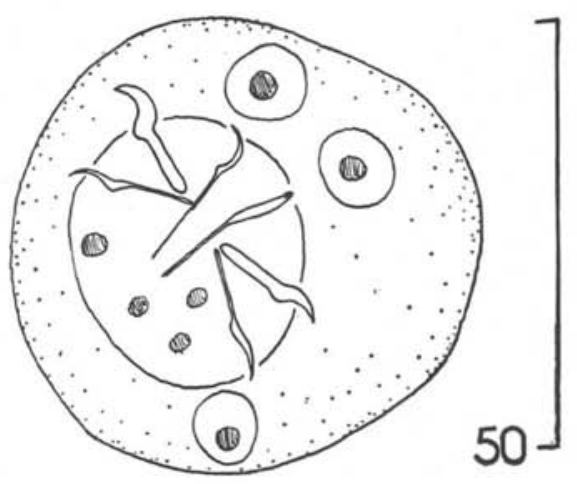

Fig. $-8 .-H$. (H.) amphitricha. CEuf.

2) HYMENOLEPIS (HYM.) ANNANDALEI Southwell, 1922, spec. inq.

= Dicranotaenia a. López-Neyra, 1942 ; Limnolepis a. Spassky et Spasskaïa, 1954. Région indienne (Indes).

Hôte : Limosa belgica (Gmelin) [= Limosa limosa (L.)].

Caractéristiques principales : STROBILE : $6-10 \mathrm{~cm} \times 1,5-2 \mathrm{~mm}$. CROCHETS chéliformes $1 . X .32 \mu(2)$, lame $>$ garde ; disposition TESTICUles et OVAIRE du type B. POCHE DU CIRRE courte : $180 \times 40 \mu$; vAGIN musculeux volumineux : $450 \times 10-50 \mu$. Absence de réceptacle séminal différencié. $\mathrm{PC} / \mathrm{AM}=1 / 6$ à 1/10. Eurs mûrs inconnus.

Cette espèce aurait été revue à deux reprises en France : chez un Tringa sp. des environs de Marseille, par Joyeux et Baer (1939) ; les crochets légèrement plus petits, la longueur de la poche du cirre $(400 \mu$ au lieu de 180$)$ la fit décrire momentanément par les auteurs, en tant que nouvelle variété longosacco, jusqu'à ce qu'en 1940 un Scolopax rusticola L. de Corse leur fournisse des exemplaires à crochets et à poche du cirre de taille intermédiaire entre la variété et l'espèce type.

La comparaison des terminaisons génitales des trois espèces en cause semble nécessaire pour confirmer définitivement ces diagnoses, des variations de la longueur de la poche du cirre aussi considérables étant assez exceptionnelles pour une espèce donnée ; en dépit des apparences, il est possible que des crochets identiques puissent armer des espèces différentes. Ces difficultés s'expliquent dans une certaine mesure par les imprécisions de la description originale. Un vagin aussi hypertrophié et « très musculeux » est très inhabi-

(2) $1 . X .32 \mu$ signifie : une couronne de 10 crochets mesurant $32 \mu$. 
tuel, surtout en comparaison d'une poche du cirre aussi réduite ; l'expérience nous enseigne que la conformation vaginale s'adapte très souvent à celle du cirre qu'elle doit recevoir; aussi suggère-t-elle une possible confusion par Southwell des conduits sexuels mâles et femelles. De toute manière, l'absence d'un réceptacle séminal non différencié du vagin est une monstruosité improbable dans le genre Hymenolepis qui justifierait la création d'un genre distinct. Notons enfin que les mensurations du texte ne correspondent pas exactement aux proportions des figures.

Nous plaçons en conséquence cette espèce en species inquirenda (3).

\section{3) HYMENOLEPIS (HYM.) BRACHYCEPHALA (Creplin, 1829)}

$=$ Hymenolepis (Drepanidotaenia) b. Cohn, 1901; Drepanidotaenia b. LópezNeyra, 1942 ; Tschertkovilepis b. Spassky et Spasskaïa, 1954.

Région paléarctique: Europe (Slesvig, Poméranie; Hollande (Cohn), et Afrique (Egypte).

Hôte: Philomachus pugnax (L.).

Caractéristiques principales (d'après Krabbe, 1869 et Cohn, 1901 dont la redescription, essentiellement à base d'interprétation de coupes histologiques, manque de rigueur).

Strobile: $70 \times 1,5 \mathrm{~mm}$; Crochets claviformes $1 . X .54-57 \mu$. Musculature longitudinale interne du strobile : 20-30 faisceaux. Atrium GÉNITAL dextre, dépourvu de sacculus, armé de nombreuses épines fines. PocHE DU CIRRE peu musculeuse, $225 \times 75 \mu$ (?); $\mathrm{PC} / \mathrm{AM}=1 / 4$ (?). CIRre court et fin $(50 \times 5 \mu)$, glabre. Disposition Testicules-ovaire du type G (ovaire en arrière des testicules). VAGIN tubulaire de $175 \mu$. UTÉRUS sacculaire. CEUFS de $25 \mu$ de diamètre (?). Testicules non persistants.

\section{4) HYMENOLEPIS (HYM.) CAMBRENSIS Davies, 1939}

$=$ Nadejdolepis c. Spassky et Spasskaïa, 1954. L.

Région paléarctique: Europe (Grande-Bretagne). Hôte: Haematopus ostralegus

Matériel observé : 6 strobiles constituant les types de Davies.

\section{Caractéristiques principales et complément d'information :}

Strobiles : $4,5 \times 0,46 \mathrm{~mm}$. Scolex : $150 \times 210 \mu$ de diamètre. Lorsque le rostre est invaginé, l'extrémité antérieure du scolex est conique et particuliè-

(3) Spassky et Spasskaïa (1954), l'ont malheureusemenu choisi pour type du genre Limnolepis. 
rement effilée (180-230 $\mu$ mesurés à partir du bord antérieur des ventouses $\times 25 \mu$ de diamètre minimal).

CROCHETs claviformes; leur forme véritable est celle que nous figurons (fig. 9) ; elle est donc classique. Leur mensuration n'atteint que $46-51 \mu$ (et non pas $53 \mu$ ) $\times 6-7 \mu$ de hauteur.

CANAUX eXCRÉTEURS : position analogue à celle décrite et figurée chez $H$. lauriei et $H$. charadrii (Ann. Parasit. hum. et comp., 1960,35 , p. 542 et 548 ).

MUSCULATURE LONGITUDiNALE interne du strobile: mal perceptible sur les types; vraisemblablement 4 bandes par face.

ATRIUM GÉNITAL: absence d'armature et de sac accessoire.

PoCHE DU CIRRE : $160 \times 16 \mu . \mathrm{PC} / \mathrm{AM}=1 / 1,84$.

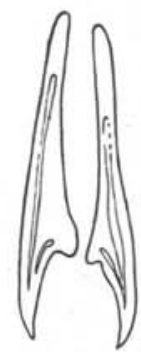

25

Fig. 9. $-H$. $(H$. cambrensis Davies, 1939. Haematopus ostralegus L. Types. Crochets du rostre.

CONDUITS GÉNITAUX :

a) CIRRE : glabre, court, fin, uniformément cylindrique, $23-25 \times 1,3-2 \mu$ de diamètre. S'insère sur une sorte d'ampoule creuse chitinoïde de 6-7 $\mu$ de diamètre qui clôt l'extrémité porale de la poche du cirre au fond de l'atrium.
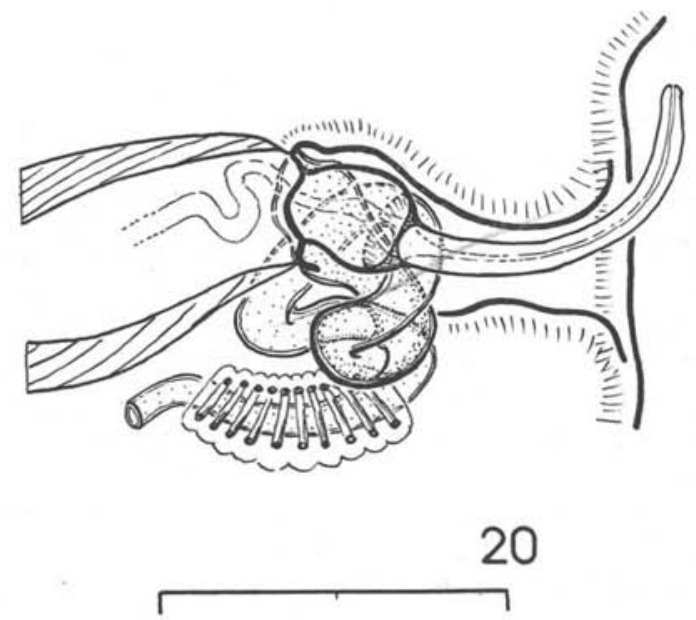

FIG. 10. - H. (H.) cambrensis: conduits sexuels terminaux et atrium génital. Vue dorsale.

b) VAGIN : long au total de $70 \mu$. Hétérogène : portion copulatrice en forme de tube chitinoïde de 4-6 $\mu$ de diamètre, étroitement pelotonné du côté ventral par rapport à l'atrium, sur une longueur de $40 \mu$ environ (4). Il s'engage ensuite, sur une longueur d'une quinzaine de microns, dans l'axe d'un tunnel musculaire légèrement arqué, formé de la juxtaposition d'une dizaine de fibres annulaires parallèles situées postérieurement à l'atrium. Il aborde finalement le réceptacle séminal après un dernier parcours de $20 \mu$ environ. Absence de fibres rétractrices (fig. 10).

Disposition testicules-ovaire de type G.

(4) Aux faibles grossissements du microscope, la région atriale ne montre que 4-5 sphérules juxtaposées; ils représentent en fait les sections optiques dorso-ventrales du vagin distal et de l'ampoule basale du cirre. 
5) HYMENOLEPIS (HYM.) CAPELLAE Baer, 1940

$=$ Dicranotaenia $c$. Skriabine et Mathevossian, 1945 ; Limnolepis $c$. Spassky et Spasskaïa, 1954.

Région néotropicale : Antigua (Antilles). Hôte : Capella delicata (Ord.).

Caractéristiques principales : STROBILE : $30 \times 1 \mathrm{~mm}$; CROCHETS chéliformes 1.X.18-21 $\mu$; garde de $15 \mu$ plus courte que la lame. Musculature non décrite. Atrium dépourvu d'armature et de sacculus. Poche du CIRRE : 234$252 \times 36 \mu . \mathrm{PC} / \mathrm{AM}=1 / 1,8$, d'après la figure. CIRRE finement épineux (90$100 \mu$ ). Disposition ovaire-Testicules de type B. Vagin non décrit. Euf de $35 \mu$ de diamètre.

La description de $H$. capellae est insuffisamment détaillée pour que l'on puisse assimiler sans réticence cette espèce américaine à l'Hymenolepis d'un Capella gallinago décrit en Pologne par Rybicka en 1958, dont la morphologie générale s'en rapproche sans s'y superposer exactement.

6) HYMENOLEPIS (HYM.) CAPILLAROIDES (Fuhrmann, 1906)

$=$ Dicranotaenia $c$. López-Neyra, 1942 ; Dubininolepis $c$. Spassky et Spasskaïa, 1954.

L'espèce observée chez un Capella sp. des Indes par Southwell en 1922 doit vraisemblablement être, pour des raisons de spécificité parasitaire, différente des parasites de Podicipédiformes et de Colymbiformes d'Amérique décrits par Fuhrmann.

Aucune autre espèce connue, spécifique de Charadriiformes et possédant de tels crochets, chéliformes, longs de $21 \mu$, à garde de $14 \mu$ plus courte que la lame, ne peut actuellement lui être substituée. Le reste de son anatomie étant inconnu, cette espèce ne doit plus être retenue, à notre avis, comme Hymenolepis parasite de Charadriiformes.

7) HYMENOLEPIS (H.) CHARADRII Yamaguti, 1935

$=$ Echinocotyle $c$. (?) López-Neyra, 1942 ; Nadejdolepis $c$. Spassky et Spasskaïa, 1954.

Régions paléarctiques: Europe (Grande-Bretagne: Davies, 1939; France: Deblock et coll., 1960) et Japon (Yamaguti).

Hôtes: Charadrius alexandrinus dealbatus (Swinhoe), Haematopus ostralegus L. ; en France: Charadrius hiaticula L.; Erolia ruficollis minuta (Leisler) et Erolia alpina (L.) (Baie de Canche). 


\section{Description de l'espèce.}

Correspond à celle des Cestodes de la même espèce parasitant les Lari [cf. Ann. Parasit. hum. et comp., 1960, 35 (4), p. 539]. Seules, les tailles des STRoBILES diffèrent. La plupart de ceux-ci sont petits : 1.400 à $2.500 \mu$ (moyenne $2 \mathrm{~mm}$ ), les largeurs maximales variant de 240 à $390 \mu$. Les chaînes complètes (à l'exception des derniers anneaux portant les œufs mûrs très rarement fixés au strobile) comportent en moyenne 47 anneaux (extrêmes : 33 à 60) : 12 à 20 ANNEAUX IMMATURES (cou + anneaux mâles) ; 8 à 12 ANNEAUX Mûrs (hermaphrodites) et 13 à 20 gravides. Néanmoins, il existe aussi des exemplaires nettement plus longs (jusqu'à 7-8 mm). CROCHETs falciformes : 1.X.57-64 $\mu$. Musculature longitudinale interne de 4 faisceaux par face. ATrIUM GÉNITAL : dextre, inerme, dépourvu de sacculus. PochE DU CIRRE très musculeuse : $120 \times$ $25 \mu . \mathrm{PC} / \mathrm{AM}=1 / 1,7$. CIRre évaginé court (aspect le plus fréquent) : 25-30 $\times 6-9 \mu$, finement armé ; VAGIN de $80-90 \mu$, droit, à dilatation chitinoïde cylindrique. Testicules non persistants. Disposition ovaire-Testicules de type G.

ANNEAUX GRAVIDES: l'élimination des œufs se fait collectivement dans le tube digestif de l'hôte, sous forme de masses ovoïdes d'œufs agglomérés, de 300-380 × 230-260 $\mu$ de diamètre, représentant le sac utérin libéré de l'anneau gravide. La coque interne de l'œuf de ces agglomérats mesure 30-35 $\times$ 20-25 $\mu$, la coque externe $50-70 \times 50 \mu$. Les crochets de l'embryon sont hétérogènes, les crochets internes des paires latérales étant plus développés que les quatre autres.

\section{8) HYMENOLEPIS (HYM.) CHIONIS Fuhrmann, 1921, sp. inq.}

= Dicranotaenia c. López-Neyra, 1942 et Skriabine et Mathevossian, 1945 ; Limnolepis c. Spassky et Spasskaïa, 1954.

Localisation géographique : Iles Kerguelen.

Hôte : Chionis alba (Gmel.).

\section{Caractéristiques principales :}

Strobile : $20 \times 0,7 \mathrm{~mm}$. Crochets chéliformes à garde massive : 1.X.21$23 \mu$. Lame $=$ garde.

POCHE DU CIRRE : $180 \times 48 \mu$. PC/ $\mathrm{AM}=1 / 4$ à $1 / 5$ (?).

CIrre court. Position testicules de type C ou G. Reste de l'anatomie non précisé.

9) HYMENOLEPIS (HYM.) CLANDESTINA (Krabbe, 1869) Railliet, 1899

= Drepanidotaenia c. Cohn, 1904 ; Dicranotaenia $c$. López-Neyra, 1942 ; Wardium c. Spassky et Spasskaïa, 1954. 
Région paléarctique; Europe: Poméranie (Creplin in Krabbe) et Grande-Bretagne (Davies).

Hôte : Haematopus ostralegus L.

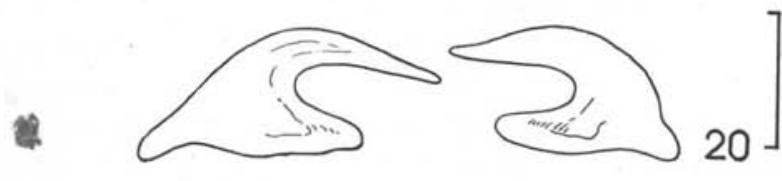

Fig. 11. - H. (H.) clandestina (Krabbe, 1869).

Haematopus ostralegus L. Grande-Bretagne. Crochets du rostre.

Matériel de redescription : 1 STrobile du matériel de Davies (1939), long de $100 \mathrm{~mm} \times 1,4 \mathrm{~mm}$ de large. Crochets chéliformes : 1.X.43-46 $\mu$ (et non pas 51-55 $\mu$ d'après Davies) $\times 19-21 \mu$ de haut; garde de 27-30 $\mu$, plus courte que la lame (fig. 11). Musculature longitudinale interne du strobile formée d'une quinzaine de larges faisceaux sur chacune des faces. ATRIUM GÉNITAL dextre, sans armature, ni sacculus. POCHE DU CIRRE musculeuse : $410 \times$ $45 \mu . \mathrm{PC} / \mathrm{AM}=1 / 3$ (1/4 dans anneaux gravides). CIRRE évaginé, long et fin : $70 \times 7-8 \mu$ de diamètre à la base et $3 \mu$ à l'extrémité ; couvert sur les $4 / 5$ de sa longueur d'un revêtement peu dense de minuscules épines de $1 \mu$ de long

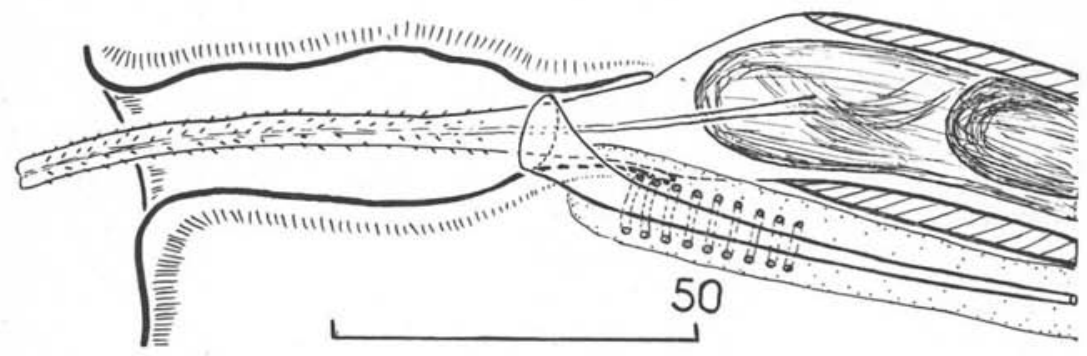

Fig. 12. $-H$. $(H$.) clandestina. - Conduits sexuels terminaux et atrium génital. Vue dorsale.

(fig. 12). Disposition ovaire-testicules de type G, avec ovaire en avant des testicules. VAGIN tubulaire long de $150 \mu$ environ dans les anneaux mûrs et de $200 \mu$ dans les anneaux gravides, de section uniforme de $5 \mu$, rectiligne ou légèrement sinueux. Sa paroi chitinoïde est mince et non plissée, sans ornementation particulière, ni différenciation décelable d'un segment initial copulateur. Toute la longueur du tube est doublée d'un manchon cellulaire qui porte son diamètre externe à $10 \mu$ et dont les $25-30 \mu$ poraux sont pourvus d'une série de fibres musculaires annulaires; absence de fibres rétractrices. Eurs mûrs inconnus. 
10) HYMENOLEPIS (HYM.) CLERCI (Shen Tseng, 1933)

$=$ Fuhrmaniella $c$. Shen Tseng, 1933 ; Hispaniolepis c. López-Neyra, 1942 et Spassky et Spasskaïa, 1954 ; Microsomacanthus c. Yamaguti, 1959. Espèce renommée Hym. shentsengi (Shen Tseng, 1933) Hughes, 1940 et Dicranotaenia vigisi Skriabine et Mathevossian, 1942 pour la différencier de Hym. clerci Fuhrm., 1924, parasite de Passériformes.

Syn. proposée : Hymenolepis (Hym.) fola Meggitt, 1933.

Région paléarctique: Chine (Pékin).

Hôte: Rhynchea capensis L.

\section{Caractéristiques principales :}

Strobile: $60 \times 1 \mathrm{~mm}$. Crochets claviformes, $1 . \mathrm{X} .27-29 \mu$.

Poche du CIRre : $396 \times 60 \mu . \mathrm{PC} / \mathrm{AM}=1 / 2$ (d'après la figure). Cirre épais et épineux, de $120 \mu$ au moins (d'après la figure). Disposition ovaIRETESTICUlEs de type D. RÉCEPTACLE SÉMINAL piriforme. UTÉRUS sacculaire. Euf : 48-54 $\mu$. Reste de l'anatomie non précisé.

\section{1) HYMENOLEPIS (HYM.) COLLARIELLA Coil, 1956}

= Dicranotaenia $c$. Yamaguti, 1959.

Région néarctique (Mexique).

Hôte : Charadrius collaris Vieillot.

Matériel d’observation: Type du Musée National des E.U. $\mathrm{n}^{\circ} 38.163$.

Le type d'H. collariella est constitué d'un scolex suivi d'une chaîne de 1,70 mm de 190 anneaux dont 20 mûrs et 20 anneaux gravides jeunes. Les deux fragments isolés de chaînes gravides de la préparation n'appartiennent pas à la même espèce (les cirres et les vagins sont bien différents) (5). En conséquence, la description originale doit subir d'importants amendements et les figures 2, 9 et 15 n'ont plus de valeur :

Rostre pourvu de 10 crochets (et non 8), chéliformes, de 12-14 $\mu$. Cirre petit, court, inerme, cylindro-conique à l'état évaginé, $25 \mu$ de long sur $5 \mu$ de diamètre à la base et $2,5 \mu$ à l'extrémité. POCHE DU CIRRE peu musculeuse : 245-290 $\mu \times 30-35 \mu$. Rapport PC/AM $=1 / 3,5$. VAGIN : court $(40-50 \mu)$, dépourvu de dilatation et de sphincter. RÉCEPTACLE SÉMINAL très volumineux : 270-360 × 100-140 $\mu$, et piriforme, l'extrémité effilée dépassant les C.O.R. (6)

(5) Le cirre de l'Hymenolepis sp. est long de $110-130 \mu \times 10-12 \mu$ de diamètre et pourvu de très fines soies de $2,5 \mu$; le vagin est rectiligne, long de $140 \mu \times 10-13 \mu$ de diamètre externe et sa paroi interne paraît être pourvue de papilles. Le rapport PC/AM n'est que légèrement inférieur à $1 / 2(1 / 2,5)$.

(6) COR: canaux osmo-régulateurs. 
poraux pour venir à la rencontre du vagin. OvaIRE : $340-365 \times 50-70 \mu$, légèrement en avant des testicules disposés en ligne (type $H$ 1). VITELlogène : $80 \times 50-60 . \mu$. UTÉRUS gravide jeune : sacculaire multilobé. EUFs : non présents dans la préparation.

La morphologie corrigée de ce Cestode ne permet pas de le distinguer de $H$. hughesi tel que nous le redécrivons ( $\mathrm{cf}^{\circ} \mathrm{n}^{\circ} 20$ ) ; il en constitue par conséquent un synonyme.

12) HYMENOLEPIS (HYM.) CROCETHIA (Belopolskaïa, 1953)

= Dicranotaenia $c$. Belopolskaïa, 1953 ; Nadejdolepis $c$. Yamaguti, 1959.

Région paléarctique (Sibérie orientale). Hôte : Crocethia alba (Pall.).

Matériel observé : 6 cestodes (M.M. Belopolskaïa leg.). Fig. 13 et 14.

Strobile : $20 \times 0,4 \mathrm{~mm}$. Crochets falciformes: $1 . X .102 \mu$. Ventouses inermes. Musculature longitudinale : quatre (?) bandes musculaires internes et une trentaine de fibres externes sur chacune des faces du strobile. Atrium GÉNITAL de section triangulaire à sommets arrondis ; inerme ; à sa partie postérieure, présence d'un sacculus accessorius du type " peigne atrial », en croissant largement ouvert, de $30 \times 13 \mu$, dont l'arc interne, légèrement épaissi, de 20-25 $\mu$ de long, est garni de soies longues $(10-12 \mu)$ et fines. POCHE DU CIRRE : $130-170 \times 30-35 \mu$, musculeuse. $\mathrm{PC} / \mathrm{AM}=1 / 2$ à $1 / 1,6$. CIRRE : court, claviforme $(15-25 \times 5 \mu$ de diamètre) et très finement spinulé (épines de $1 \mu$

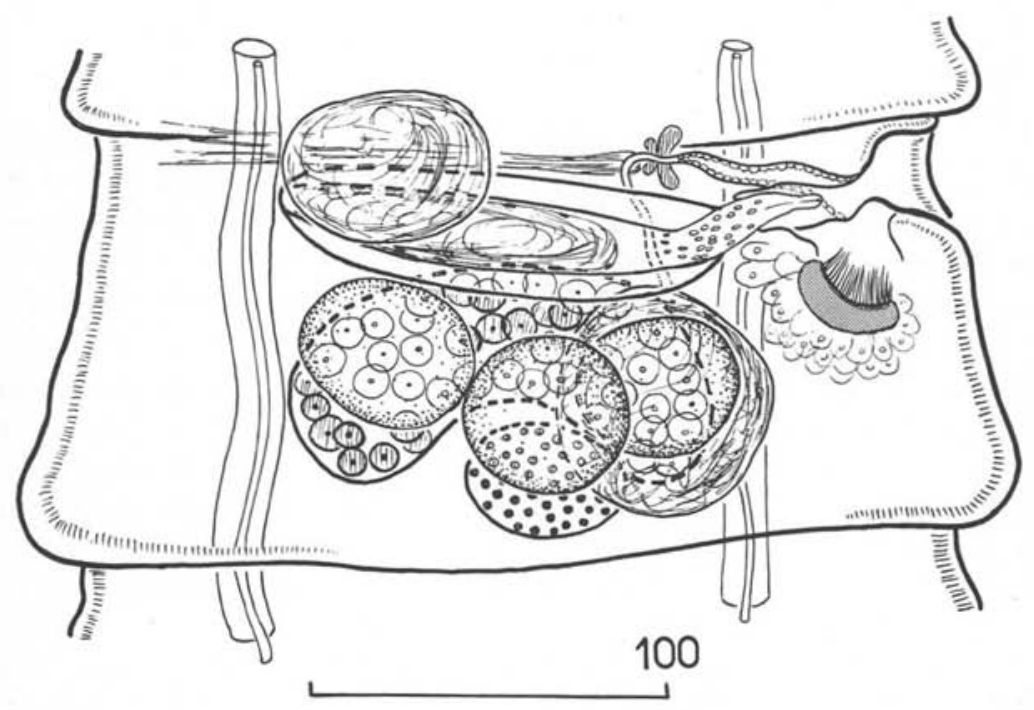

Fic. 13. - H. (H.) crocethia (Belop., 1953). Crocethia alba (Pall.). Sibérie orientale. Anneau mûr. Vue dorsale. 


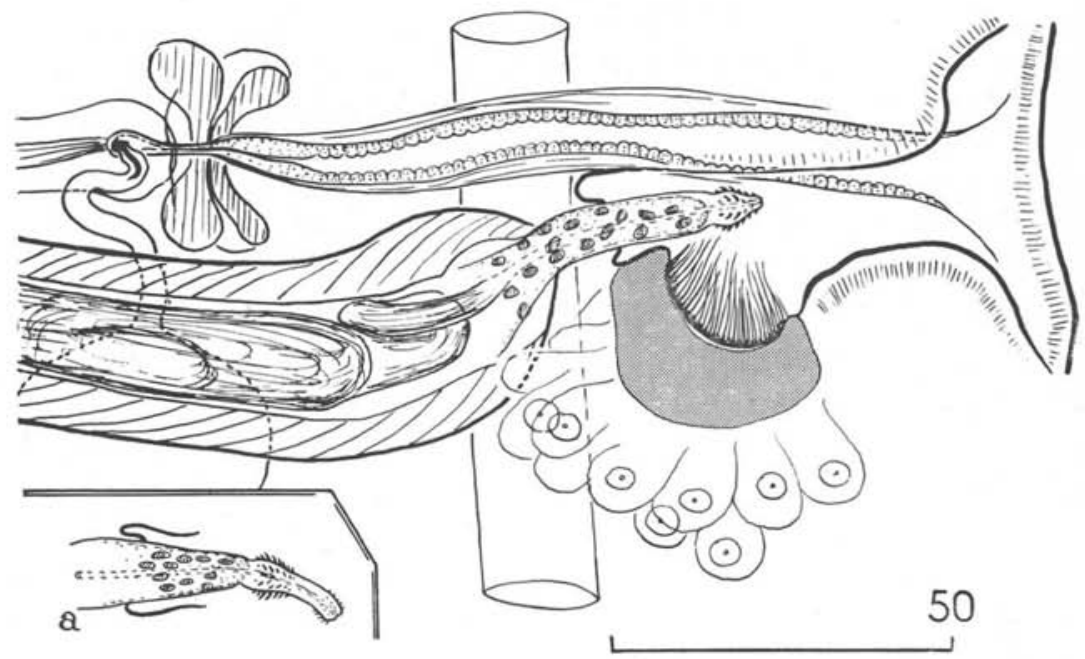

Fig. 14. $-H$. (H.) crocethia. Conduits sexuels terminaux et atrium génital. Vue dorsale. En a) autre aspect du cirre évaginé.

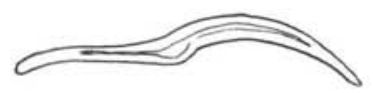

Fig. 15. $-H$. (H.) crocethiae (Webster, 1947) Coil, 1956. Type. Arenaria interpres (L.). Crochets du rostre de $95 \mu$.

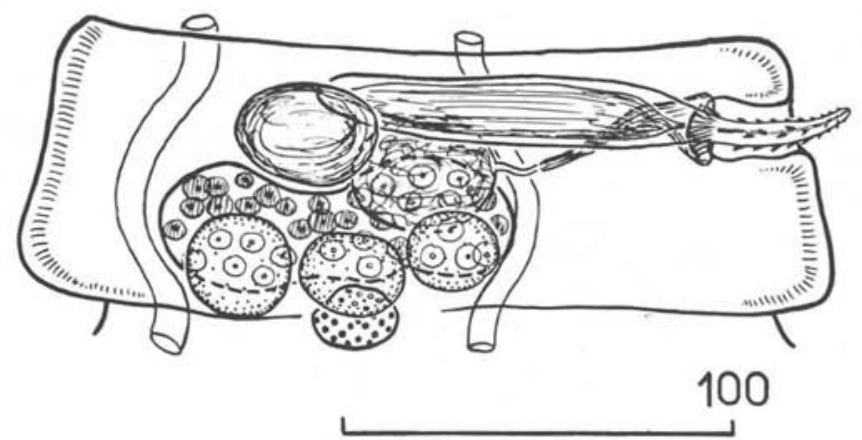

Fig. 16. $-H$. $(H$.$) crocethiae. Anneau mûr. Vue dorsale. Reconstitution synthétique.$

maximum). Totalement évaginé, il se prolonge d'une portion plus amincie, qui présente l'aspect de la figure $14 a$, portant sa longueur à $35 \mu$.

VAGIN long de $120 \mu$ au total, mais de structure hétérogène :

1) Segment copulateur en forme de cône effilé, long $(90 \times 13 \mu)$, rectiligne 
et occlus à sa pointe par un volumineux sphincter musculaire de $30 \mu$ de diamètre, en arrière duquel s'insèrent des fibres rétractrices bien figurées. Parois vaginales internes garnies de fines papilles analogues à celles de $\mathrm{H}$. mudderbugtenensis (Baer, 1956). La paroi chitinoïde se poursuit très légèrement audelà du sphincter, permettant l'insertion des muscles.

2) Segment proximal en forme de tube non chitinisé, dirigé à angle droit par rapport au segment copulateur, long de $25 \times 4 \mu$ et en relation avec le réceptacle séminal. Disposition ovaIRE-TESTICULES de type C.

$$
* *
$$

Ce Cestode, en dépit d'une homonymie regrettable, est bien différent de l'espèce suivante.

$$
* *
$$

13) HYMENOLEPIS (HYM.) CROCETHIAE (Webster, 1947), spec. inq.

$=$ Hym. (Echinocotyle $)$ c. Webster, 1947 ; Echinocotyle c. Yamaguti, 1959.

Région néarctique : U.S.A. (Webster, 1947) et Mexique (Coil, 1956).

Hôtes: Arenaria interpres (L.), Crocethia alba (Pall.).

Matériel observé : types du Musée National des E.U. n 45.746.

Caractéristiques principales (fig. 15 et 16):

Strobile : $2,3 \times 0,19 \mathrm{~mm}$; CROCHETS falciformes, $1 . X .87-93 \mu(86-98$ d'après Webster). Ventouses inermes (6). Atrium GÉNITAL: présence d'un sacculus accessorius du type "peigne atrial », largement ouvert, de 11-14 $\mu$ de long, garni de soies de 6-7 $\mu$ et situé entre l'ouverture vaginale et la base du cirre. POCHE DU CIRRE : $80-90 \mu \times 14-17 \mu$; PC/AM $=1 / 2,1$. Absence de fibres rétractrices. CIRRE : cylindro-conique, bien développé par rapport au Cestode : $40 \times 7 \mu$ à la base ; armé de courtes et fortes épines $(2,5 \mu)$ en crochets, de tailles décroissantes, sur une longueur de $25 \mu$. VAGIN : conique, de 50-60 $\mu \times 16-17 \mu$ à l'embouchure et $4 \mu$ au sommet. Paroi interne finement grenue, apparemment glabre. Dépourvu (?) de sphincter et de fibres rétractrices. Disposition ovaire-testicules de type D (?). Gufs (d'après Coil, 1956) : 19-22 $\mu$, avec expansion polaire.

\section{4) HYMENOLEPIS (HYM.) FOLA Meggitt, 1933}

$=$ Dicranotaenia $f$. López-Neyra, 1942 ; Passerilepis $f$. Spassky et Spasskaïa, 1954. Région indienne: Indes (Meggitt). Région paléarctique : Japon (Yamaguti, 1940). Hôte : Rostratula benghalensis (L.).

(6) Sub-circulaires; n'ont pas l'aspect caractéristique allongé et la platitude des ventouses du sous-genre Echinocotyle, aussi replaçons-nous ce cestode parmi les Hymenolepis vrais. 
Caractéristiques principales (selon Yamaguti, 1940): Strobile : 4,5 $\times$ $1,6 \mathrm{~mm}$. CRochets claviformes, 1.X.32 $\mu$. Musculature longitudinale interne : $2 \times 4$ faisceaux. Atrium géNITAL inerme, dépourvu de sacculus. PochE DU CIRRE musculeuse : $400-500 \times 60-80 \mu$. $\mathrm{PC} / \mathrm{AM}=1 / 3$ (d'après la figure). CIRre évaginé : $200 \times 27 \mu$, armé de soies. Disposition OVAIRE-TESTICUles de type C. VAGIN distal : $300 \times 15-20 \mu$. Eufs non décrits.

Remarque. - Il semble qu'une synonymie avec Hym. (H.) clerci Shen Tseng, 1933 puisse être confirmée lors de nouvelles récoltes de ces deux espèces.

\section{5) HYMENOLEPIS (HYM.) GLANDULARIS Fuhrmann, 1909}

= Weinlandia g. Mayhew, 1925; Orlovilepis g. Spassky et Spasskaïa, 1954 ; Cloacotaenia g. Yamaguti, 1959.

Région paléarctique: Afrique (Egypte). Hôte: Himantopus himantopus (L.). Parasite du rectum.

Caractéristiques principales : Strobile : 3 à $4 \mathrm{~mm}$. Anneaux larges et courts $(30-40 \mu)$, de maturation rapide. Gros SColex, de $1.250 \mu$ de diamètre. Petit rostre inerme de $110 \mu$ de diamètre, entouré de glandes. VENTOuses de $570 \mu$, inermes. Musculature interne du strobile vraisemblablement formée de plus de 4 faisceaux par face. Disposition ovaIRE-TESTICUles probablement du type C. Poche DU CIRRE atteignant les canaux osmo-régulateurs antiporaux. CIRRE long et épais. UTÉrus sacculaire ne contenant généralement qu'une seule rangée d'çuFs de $37 \mu$ de diamètre. Reste de l'anatomie et autres mensurations inconnus.

16) HYMENOLEPIS (ECHINOCOTYLE) GLAREOLAE (Singh, 1952)

$=$ Echinocotyle g. Singh, 1952.

Région indienne (Indes). Hôte : Tringa glareola L. Type non consultable en dehors du pays d'origine.

Caractéristiques principales : STROBLL : 2,2-3,1 $\times$ 0,19-0,23 mm. CroCHETs falciformes, 1.X.65-72 $\mu$. VEnTouses très ovalaires, épineuses. ATRIUM GÉNITAL armé et pourvu d'un sac accessoire de $39 \times 10 \mu$. Musculature du strobile non décrite. Poche DU CIRre musculeuse, de $166 \times 31 \mu$. PC/ AM $=$ $1 / 2$ à $1 / 2,5$ (d'après la figure). Cirre long, effilé et inerme, de $78 \times 10 \mu$. VAgIN tubulaire. Disposition ovaire-testicules de type D. Utérus sacculaire. Eufs de 13-15 $\mu$ de diamètre.

Remarque. - Cette espèce ne se distingue pratiquement de Hymenolepis (Echinocotyle) nitida Clerc, 1902, tel que nous le redécrivons ( $\mathrm{n}^{\circ} 33$ ), que par 
la différence de taille des crochets du rostre. La synonymie avec $H$. nitidulans (Krabbe, 1882), proposée par Baer (1956, p. 51), ne semble pas devoir être retenue.

17) HYMENOLEPIS (H.) GUSCHANSKOI (Krotov, 1952)

= Dicranotaenia $\mathrm{g}$. Krotov, 1952.

Région paléarctique: Sakhaline (Krotov, 1952) et côtes de la mer du Japon (Belopolskaïa, 1953 et 1959).

Hôtes : Calidris minuta Leisler ; Calidris ruficollis (Pallas), Crocethia alba (Pall.), Erolia alpina (L.) Belopolskaïa.

Matériel observé : deux Cestodes complets de Calidris ruficollis (M. M. Belopolskaïa leg.) (fig. 17 et 18).

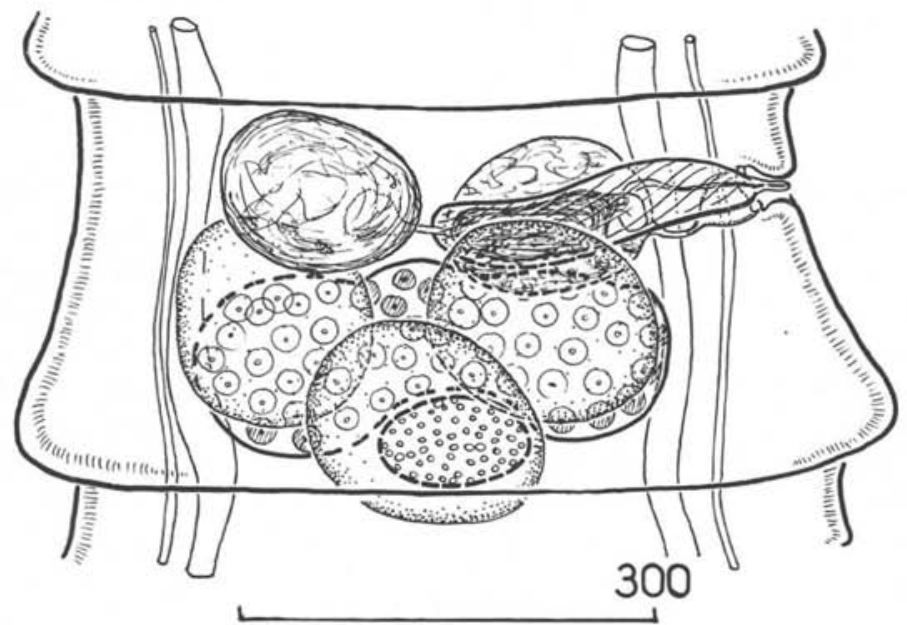

Fig. 17. $-H$. (H.) guschanskoi (Krotov, 1952). Calidris ruficollis (Pall.). Sibérie orientale. Anneau mûr. Vue dorsale.

Strobile : $40 \times 64 \mathrm{~mm}$. Crochets falciformes: $1 . X .85-87 \mu$ (7). Musculature longitudinale du strobile formée de 20-25 fines fibres externes et d'une douzaine de fibres internes plus volumineuses sur chacune des faces du Cestode.

ATRIUM GÉNITAL : de section triangulaire, à sommets arrondis ; non épineux, dépourvu de sacculus accessorius.

POCHE DU CIRRE : $215 \times 40 \mu$. PC/ $\mathrm{AM}=1 / 3,2$ à $1 / 2,5$.

CIRRE : long et fin ( $80-90 \mu \times 8 \mu$ à la base), à l'état d'évagination maximale. De structure hétérogène: 1) une base glabre, de $20 \times 8 \mu$; 2) une portion

(7) $89-92 \mu$ d'après Krotov. 
finement épineuse (épines de $1 \mu$ ), sur une longueur de $20 \mu$. Ces deux premiers segments sont seuls observables sur les cirres partiellement évaginés ; 3) un segment de $25 \mu$ garni d'épines fines et longues comme des soies $(4 \mu)$; 4) un segment terminal en stylet fin et glabre, de $20 \times 2 \mu$ de diamètre.

VAGIN : long de $90 \mu$ environ; de structure hétérogène : $1^{\circ}$ portion copulairice conique, longue de 40-50 $\mu \times 10-13 \mu$ de diamètre maximal, à parois chitinoïdes, se terminant à la pointe du cône par un tortillon spiralé étroit de $15 \mu$ de long et entouré de fibres musculaires annulaires obliques ; $2^{\circ}$ partie proximale droite, longue de $25 \mu$, située dans le prolongement du vagin, en rapport avec le réceptacle séminal. Ni papille, ni fibres rétractrices.

Disposition ovaIRE-TESTICUles de type D.

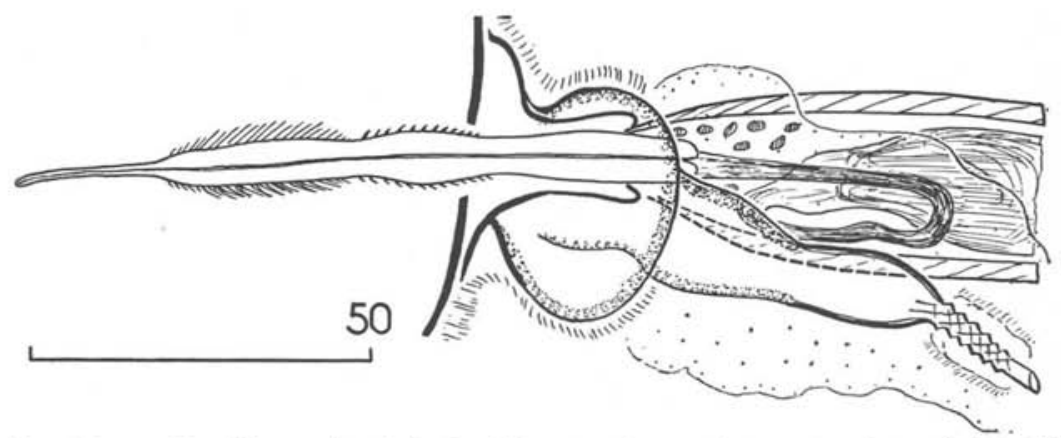

FIg. 18. - H. (H.) guschanskoi. Conduits sexuels terminaux et atrium génital. Vue ventrale.

Eufs mûrs : ovoïdes ; coque externe : 60-64 × 50-53 $\mu$, uniformément mince ; coque interne accolée à l'embryon : $33 \times 28 \mu$. Crochets de même longueur $(11,5 \mu)$, mais hétérogènes, les crochets internes des paires latérales étant plus développés que les quatre autres.

18) HYMENOLEPIS (HYM.) HAMASIGI Yamaguti, 1940

$=$ Dicranotaenia $h$. Skriabine et Mathevossian, 1945 ; Limnolepis $h$. Spassky et Spasskaïa, 1954.

Région paléarctique (Japon).

Hôte : Erolia alpina sakhalina (Vieillot).

Caractéristiques principales : Strobile : $130 \times 0,6-1,75 \mathrm{~mm}$. Crochets chéliformes : $1 . X .33 \mu$; lame longue de 24-25 $\mu$, dépassant la garde. Musculature longitudinale interne de 15 à 20 faisceaux. ATRIUM GÉNITAL inerme dépourvu de sacculus. Poche du CIRRE non musculeuse : 320-400 $\times$ 40-70 $\mu$. Cirre fortement armé (épines de $9 \mu$ ) : $180 \times 27 \mu$. PC/ $\mathrm{AM}=1 / 3$. VagiN 
tubulaire étroit de $200 \mu$ environ. Disposition ovaire-testicules de type B. Eufs mûrs inconnus.

19) HYMENOLEPIS (HYM.) HIMANTOPODIS (Krabbe, 1869), sp. inq.

$=$ Dicranotaenia $h$. López-Neyra, 1942 ; Wardium $h$. Spassky et Spasskaïa, 1954. Syn. (?) : H. recurvirostrae (Krabbe, 1869) Baer, 1959, p. 103.

Région paléarctique: Europe (Krabbe) ; région indienne (Indes; Singh 1960).

Hôtes: Himantopus melanopterus (Bremser); Him. himantopus (L.).

Cette espèce n'est bien connue que par ses crochets chéliformes de 8 à $9 \mu$, à lame plus courte que la garde (épaisse et spatulée d'après Baer, 1940); elle ne possède pas d'iconographie, ni de description d'ensemble précise, bien qu'elle ait été revue à quatre reprises: $1^{\circ}$ par Meggitt, 1927 (Recurvirostra avosetta L. - Egypte) ; $2^{\circ}$ par Shen Tseng, 1933 (Tringa totanus (L.) - Chine) ; $3^{\circ}$ par Joyeux et Baer, 1936 (réexamen des types de Krabbe) (?) ; $4^{\circ}$ par Baer, 1940 (Himantopus mexicanus Mull. - Antilles) (7 bis).

Il s'agirait de Cestodes de 50-70 mm $\times 1-1,5 \mathrm{~mm}$, à Testicules du type $\mathrm{G}$ (en ligne). Deux descriptions soulèvent néanmoins le problème de l'identité réelle des quatre espèces signalées:

$1^{\circ}$ Les CROCHETs de l'espèce égyptienne ont * une silhouette légèrement différente » de celle figurée par Krabbe, de l'aveu même de Meggitt.

$2^{\circ}$ La POCHE DU CIRRE (supérieure à $90 \mu$ dans les anneaux mûrs) s'étend presque jusqu'aux C.O.R. antiporaux chez l'espèce de Meggitt, alors qu'elle n'atteint que la moitié de la largeur totale dans la description de Shen Tseng (ce qui, pour un anneau de $1.000 \mu$, doit néanmoins lui conférer une taille d'au moins $500 \mu$; or, Joyeux et Baer ne lui en accordent que $280 \mu$ ). Ces derniers ont observé, en plus, des fibres rétractrices puissantes sous-tendant les conduits génitaux $\delta^{*}$ et $q$, et un sphincter vaginal. Baer, en 1940, ne semble avoir basé sa détermination que sur l'armature du rostre.

Des connaissances aussi diffuses ne peuvent que suggérer la temporisation en ce qui concerne la synonymie avec les Cestodes du \& complexe » recurvirostrae (cf. plus loin $\mathrm{n}^{\circ} 42$ ).

\section{0) HYMENOLEPIS (HYM.) HUGHESI Webster, 1947}

$=$ Variolepis $h$. Spassky et Spasskaïa, $1954 ;$ Hybridolepis h. Spassky, 1959.

Syn. : Hymenolepis collariella Coil, 1956.

Région néarctique (U.S.A., Texas).

Hôte : Charadrius melodus Ord.

Matériel d'observation: Type du Musée National des E.U. $\mathrm{n}^{\circ} 45.744$.

(7 $7^{\text {bs }}$ ) L'article de Singh (Indian Jl Helm. 1959, 11, 43-62) est parvenu trop tard a notre connaissance et n'a pu être analysé. 
Le type d' $H$. hughesi est constitué d'un scolex suivi d'une chaîne de $11 \mathrm{~mm}$, de 190 anneaux, dont 70 mâles et 10-15 mûrs; aucun n'est gravide ; les deux fragments de chaînes (un mûr et l'autre gravide) de la préparation n'appartiennent pas à l'espèce pourvue de scolex : la poche du cirre, très courte, n'a pas l'aspect des poches du cirre du genre Hymenolepis (vésicule séminale interne tubulaire plus ou moins sinueuse, anneau réduit de fibres musculaires circulaires à la partie moyenne de la poche); de toute manière, elle est bien distincte de celle de la partie initiale du strobile (8).

En conséquence, la description originale de $H$. hughesi doit subir d'importants amendements; les figures originales 4 et 5 (p. 106) se rapportent au faux Hymenolepis dont il est difficile de préciser l'appartenance générique exacte.

\section{Redescription de l'espèce.}

Cestode de $11 \mathrm{~mm}$, sans anneau gravide $\times 0,8 \mathrm{~mm} ; 190$ anneaux, y compris ceux du cou.
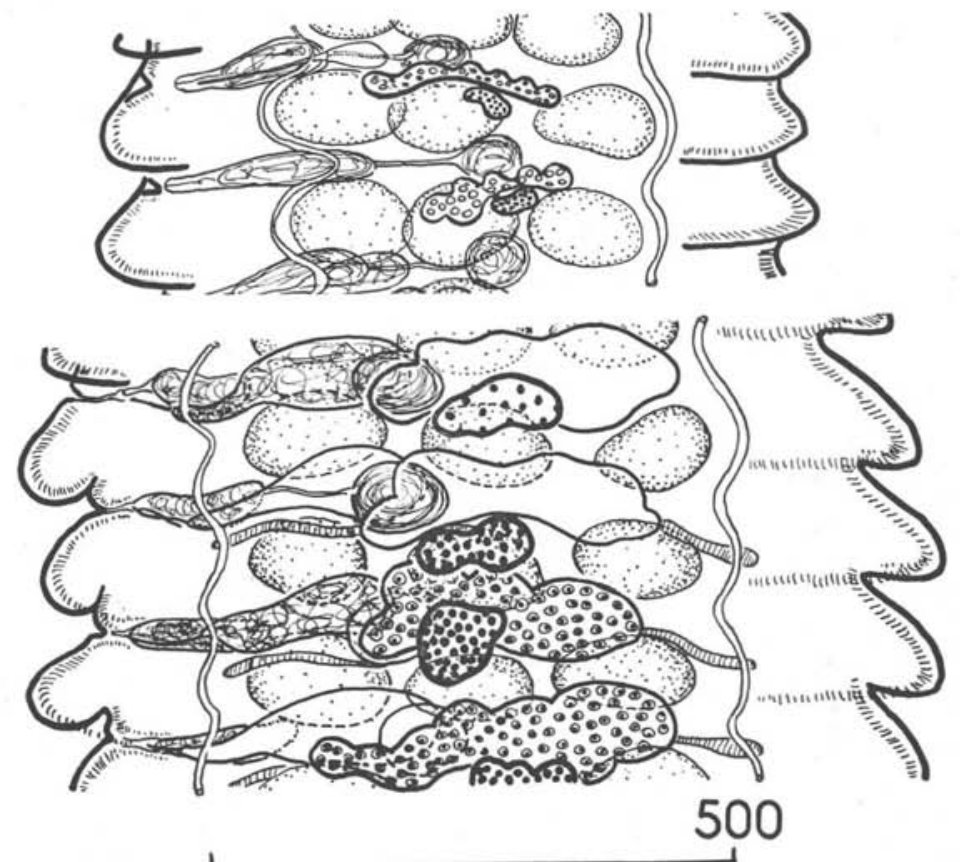

Fig. 19. - H. (H.) hughesi (Webster, 1947). Type. Charadrius melodus. Ord. Anneaux mâles. - Fig. 20. $-H$. $(H$.) hughesi : anneaux mûrs. Vues ventrales.

(8) Cette dualité explique sans doute lalternance des pores génitaux et la présence éventuelle, d'un quatrième testicule dans certains anneaux, signalées par l'auteur et que nous avons revues. Aucun de tous les fragments ne présente de Sacculus accessorius. Le genre Hybridolepis perd donc son espèce type. 
ANNEAUX : anneau mâle : $675 \times 100 \mu$; anneau hermaphrodite mûr : $800 \times$ $115 \mu$ (fig. 19 et 20 ; voir aussi fig. 51 à 54 ).

Scolex : mal conservé. Ventouses : 75-80 $\mu$ de diamètre. Rostre : $130 \times$ $56 \mu$ de diamètre. Vraisemblablement 10 crochets chéliformes de $13 \mu$; garde $>$ lame.

Cou bien marqué ; long de $350-400 \mu$ jusqu'à la première segmentation $\times$ $90 \mu$ de large.

Musculature longitudinale : une trentaine de fines fibres externes et une quinzaine de fibres internes sur chacune des faces du strobile.

CANAUX OSMO-RÉgulateurs : dépourvus d'anastomoses transverses. Ventraux par rapport aux conduits génitaux.

Atrium génital : dépourvu de sac accessoire et d'épines. Court $(20 \mu)$. Dextre.

Système mâle (dans anneaux mûrs). Testicules: au nombre de trois, sensiblement égaux, ovalaires, disposés en ligne à la partie postéro-dorsale du proglottis. Taille maximale : $100-120 \times 75-90 \mu$.

Poche DU CIRRE. Taille: $130-160 \times 30-40 \mu$ de diamètre. N'atteint jamais l'axe longitudinal médian du strobile, ne dépassant que légèrement les C.O.R. poraux. Parois minces $(2,5 \mu)$. Rapport: $\mathrm{PC} / \mathrm{AM}=1 / 5$.

CIRre : petit et court, inerme, cylindro-conique à l'état évaginé.

VÉSICUle SÉminAle externe subsphérique : $55 \mu$ de diamètre ou $65 \times 80 \mu$, en position dorsale, un peu en-deçà du niveau de l'axe longitudinal du strobile, reliée à la partie terminale de la poche du cirre par un canal déférent externe assez long.

\section{Système femelle:}

OvaIre: allongé, multilobé, $310 \times 40-45 \mu$. Prend naissance le long du bord antérieur des testicules, la glande vitellogène située de préférence entre les testicules antiporaux. Dans les anneaux mûrs, la position antéro-ventrale par rapport aux testicules est conservée (type $\mathrm{H} 1$ ).

Vitellogène : ovoïde, parfois légèrement lobé, 65-100 $\mu \times 45-50 \mu$. Situé sous le testicule médian ou entre les deux testicules antiporaux.

RÉCEPTACLE SÉmiNAL: vaste, nettement piriforme, $210-240 \times 65 \mu$. Situé ventralement et parallèlement à la poche du cirre, l'extrémité antiporale sous la vésicule séminale externe, et l'extrémité porale effilée dépassant assez souvent les canaux osmo-régulateurs poraux; le vagin la prolonge.

VAGIN : ventral, court, 50-70 $\mu$ sur 8-10 $\mu$ de diamètre externe et 3-4 $\mu$ de diamètre interne. Parois chitinoïdes dépourvues de dilatation et de sphincter. Absence de fibres rétractrices.

UTÉRUS et đEFs inconnus. 
L'examen comparé des types et les deux descriptions amendées de $H$. collariella et de $H$. hughesi ne permettent plus de séparer les deux espèces; nous les faisons tomber en synonymie.

$$
* *
$$

21) HYMENOLEPIS (ECHINOCOTYLE) HYPOLEUCI (Singh, 1952)

$=$ Echinocotyle $h$. Singh, 1952.

Syn. : Hymenolepis uralensis (Clerc, 1902) Baer, 1956 (p. 50, note 1).

Région indienne (Indes).

Hôte: Actitis (= Tringa) hypoleucos (L.).

Types non consultables en dehors du pays d'origine.

\section{Caractéristiques principales :}

Strobile : $72 \times 1,9 \mathrm{~mm}$. Scolex : $260-310 \mu$ de $\emptyset$. Crochets claviformes, 1.X.58-62 $\mu$. Ventouses ovalaires peu profondes, irrégulièrement épineuses. Atrium GÉNITAL armé muni d'un sac accessoire épineux. Musculature longitudinale interne non étudiée. POCHE DU CIRRE non musculeuse : 340-350 $\times$ $67 \mu . \mathrm{PC} / \mathrm{AM}=1 / 3,5$ (d'après la figure). CIRRE fin et glabre $(330 \mu)$. Ovaire lobé postérieur aux testicules et très allongé, dépassant les canaux osmo-régulateurs longitudinaux. Disposition OVAIRE-TEsticules du type H2. Utérus sacculaire. EuFs de $35 \mu$.

Remarques. - Le diamètre basal du cirre $(48 \mu)$ semble incompatible avec la description d'un cirre fin et avec sa figuration, p. 52, où il ne paraît pas dépasser une vingtaine de microns. Le sac accessoire pourrait mesurer $50 \times$ $25 \mu$ et le vagin, $570 \mu$ environ. Le testicule médian, décrit comme parfois légèrement antérieur aux deux autres, est figuré postérieur. La redescription du scolex de Hym. (E.) uralensis par Dubinina, 1953 (fig. 12) vient sans doute confirmer la synonymie avancée par Baer, 1956.

22) HYMENOLEPIS (HYM. ?) INNOMINATA Meggitt, 1927, spec. inq.

$=$ Microsomacanthus $i$. López-Neyra, 1942 et Spassky-Spasskaïa, 1954.

Région paléarctique: Afrique (Egypte). Hôte: Recurvirostra avosetta L.

Le seul caractère d"identification de cette espèce réside dans la taille et la forme des crochets. Strobile : $10 \times 1 \mathrm{~mm}$; crochets claviformes, 1.VIII.40$43 \mu$. Disposition ovaire-testicules de type C. Peut-être est-elle synonyme de Hym. (H.) rectacantha Fuhrm., 1906. 
23) HYMENOLEPIS (HYM. ?) INTERRUPTA (Rud., 1802)

Fuhrm., 1906, spec. inq.

$=$ Weinlandia $i$. Mayhew, 1925; Hispaniolepis i. López-Neyra ; Passerilepis $i$. Spassky et Spasskaïa, 1954; Mayhewia i. Yamaguti, 1959.

Région paléarctique (Europe). Hôtes: Lymnocryptes gallinula (L.) et Scolopax rusticola $\mathrm{L}$.

Le seul caractère d'identification de cette espèce réside dans la taille et la forme des crochets. Strobile: $100 \times 0,7 \mathrm{~mm}$. Crochets claviformes, 1.X. $27 \mu$. Poche du Cirre de $200 \mu$. Disposition ovaire-testicules de type B.

24) HYMENOLEPIS (HYM.) LAURIEI Davies, 1939

$=$ Nadejdolepis $l$. Spassky et Spasskaïa, 1954.

Région paléarctique: Europe occidentale (Grande-Bretagne) ; Sibérie orientale, Belopolskaïa, 1959.

Hôtes: Haematopus ostralegus L., Crocethia alba Pall. et Tringa totanus (L.).

La morphologie de ce Cestode correspond à celle de la publication de Davies et au complément de description de 1960 (Deblock et coll.).

Caractéristiques principales : Strobile : $3-3,5 \times 0,39-0,42 \mathrm{~mm}, 35-45$ anneaux immatures, 15-20 anneaux mûrs et 20-30 anneaux gravides. CROCHETs falciformes: 1.X.86-96 $\mu$. Musculature interne : 4 faisceaux longitudinaux par face. Atrium géNITAL : dextre, inerme, dépourvu de sacculus. Poche Du CirRe très musculeuse : $150 \times 34 \mu$. $\mathrm{PC} / \mathrm{AM}=1 / 1,2$ à $1 / 1,4$. CIRRE évaginé : 130-150 $\mu \times 9 \mu$, épineux à la base sur 25-30 $\mu$ de long. VAGIN tubulaire très long (160-180 $\mu)$, très sinueux, dépourvu de dilatation chitinoïde et de sphincter. Disposition ovaire-testicules de type G. Testicules non persistants. Eufs ovoïdes de 40-55 $\mu$ de diamètre.

\section{5) HYMENOLEPIS (HYM.) LITORALIS (Webster, 1947)}

Syn.: Hym. (Echinocotyle) litoralis Webster, 1947.

Région néarctique : Etats-Unis (Texas).

Hôte : Crocethia alba (Pallas).

Matériel d'observation: Type du Musée National des E.U. $\mathrm{n}^{\circ} 45.745$.

Strobile : $45-65 \times 0,9 \mathrm{~mm}$. Crochets falciformes : $1 . X .86-87 \mu$ (fig. 21 ). Ventouses inermes. Musculature interne non observée.

AtriUm GÉNITAL inerme, court, dépourvu de sacculus accessorius. 

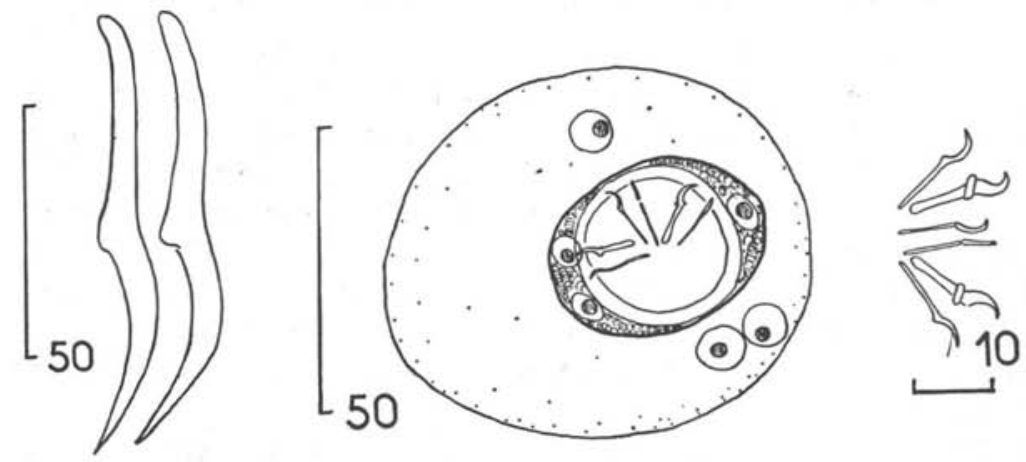

Fig. 21. $-H$. $(H$.$) litoralis (Webster, 1947). Type. Crocethia alba (Pall.). Crochets du$ rostre.

FIG. - 22. H. (H.) litoralis. Euf mûr et crochets de l'embryon.

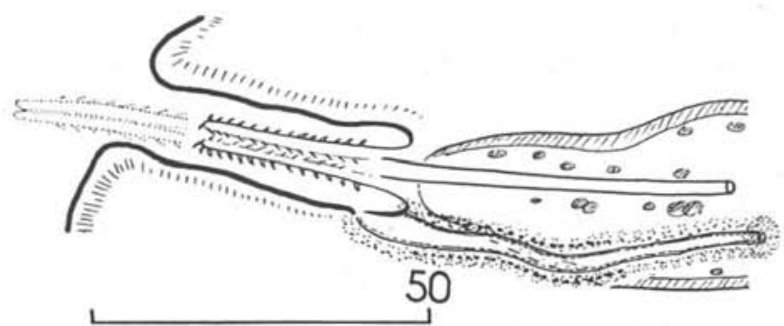

Fig. 23. $-H$. $(H$.$) litoralis. Conduits sexuels terminaux et atrium génital. Vue ventrale.$

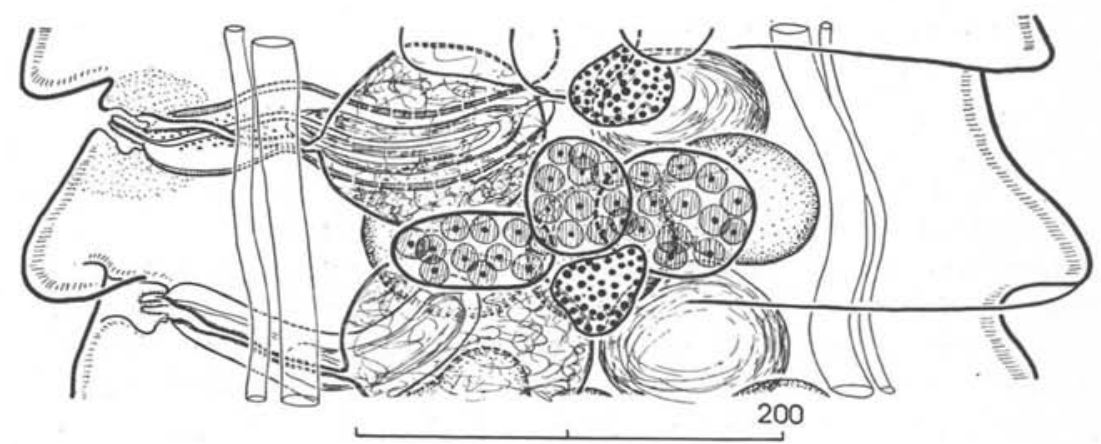

Fig. 24. $-H$. $(H$.$) litoralis. Anneau mûr. Vue ventrale.$ 
POCHE DU CIRRE : $210-250 \times 30-40 \mu$, musculeuse. $\mathrm{PC} / \mathrm{AM}=1 / 2,5$.

CIRRE : aspect fréquent de cirre court, $30-50 \mu \times 10-12 \mu$ de diamètre à la base, uniformément cylindrique et épineux (épines de $2 \mu$ de long). Prolongé dans la poche par un tube chitineux particulièrement bien visible, long de $90 \times 2,5-4 \mu$, qui, évaginé, doit former le stylet. Le cirre atteint dès lors 130$135 \mu$ de long (Webster) et ne doit être épineux qu'à sa base, sur une longueur de $50 \mu$ environ (fig. 23).

VAGIN tubulaire sans ornementation, $75-90 \mu \times 10 \mu$ de diamètre externe. Les 25 microns initiaux sont entourés de fibres annulaires discrètes. Absence de sphincter et de fibres rétractrices. Disposition ovaIRE-TESTICULES du type D ou $\mathrm{G}$ (fig. 24).

Eufs ovoïdes. Membrane externe fine : 70-80 × 50-60 $\mu$. Membrane interne légèrement plus accentuée : $40 \times 32 \mu$ ou $40 \mu$ de diamètre. Crochets de 13-14 $\mu$ d'aspect hétérogène: les crochets internes des paires latérales étant plus développés que les quatre autres (fig. 22).

26) HYMENOLEPIS LONGIOVATA Johri, 1961

Région indienne (Indes).

Hôte : Erolia ruficollis minuta (Leisler).

Publication non consultée.

\section{7) HYMENOLEPIS (ECHINOCOTYLE ?) LONGIROSTRIS}

(Rud., 1819), spec. inq.

$=$ Weinlandia l. Mayhew, 1925 ; Microsomacanthus $l$. López-Neyra, 1942 ; Nadejdolepis 1 . Spassky et Spasskaïa, 1954.

Région paléarctique : Europe (Italie).

Hôte: Glareola pratincola (L.).

Caractéristiques principales : STRoBiLe type : 3-4 à $9 \mathrm{~mm} \times 0,3$ (et non pas 40-60 mm ; cf. Baer, 1956, p. 48). CROCHETs claviformes : $1 . X .33 \mu$. Reste de l'anatomie inconnu.

Baer a trouvé une fois chez Philomachus pugnax (L.) en France (1956) et trois fois au Congo ex-belge (1959), chez Charadrius, Erolia et Hoplopterus, deux très petites espèces de Cestodes, rapportées l'une et l'autre à l'espèce de Rudolphi et dont les caractéristiques seraient les suivantes: STROBILE : $1,2 \times$ $2 \mathrm{~mm} \times$ ?. CROChets : 1 .X.35-37 $\mu$. Ventouses ovalaires à bords épineux. Atrium GÉnItAl glabre (?), pourvu d'un sac accessoire. POCHE DU CIRRE: 136-147 $\times 23-24 \mu$ ou $170 \times 40 \mu$, musculeuse. PC/ $\mathrm{AM}=$ ?. CIRRE inerme (?) à stylet, de $51 \times 2 \mu$. VAGIN à parois épaisses de conformation non précisée. Disposition ovaire-testicules non précisée, comme le reste de l'anatomie. 


\section{8) HYMENOLEPIS (HYM.) MAGNISACCIS Meggitt, 1927 nomen nudum.}

$=$ Sphenacanthus $m$. López-Neyra, 1942 ; Nadejdolepis $m$. Spassky et Spasskaïa, 1954.

Région paléarctique (Egypte). Hôte: Erolia (= Limonites) minuta Leisler.

Caractéristiques : Strobile: $17 \mathrm{~mm} \times 0,6$. Crochets falciformes: 1.X.73-75 $\mu$. Disposition ovaire-testicules du type G ou C. Poche du CirRe grande (moitié de l'anneau au moins). Reste de l'anatomie inconnu. Avec Baer, 1956, nous la considérerons comme nomen nudum (9).

29) « HYMENOLEPIS » MINOR (Krabbe, 1869) Ransom, 1909 spec. inq.

= Taenia minuta Krabbe, 1869, nec Rudolphi, 1810.

Région néarctique (Groenland). Hôtes : Phalaropus fulicularius (L.) et Phalaropus lobatus (L.) (= cinereus Briss.).

Caractéristiques: Strobile de $25 \times 1 \mathrm{~mm}$. Crochets claviformes: 1.XIV.11-12 $\mu$. Reste de l'anatomie inconnu. Sandeman (1959) ayant revu les types récemment, déplace cette espèce dans le genre Anomotaenia Cohn, 1900.

30) HYMENOLEPIS (HYM.) MOGHENSIS Inamdar, 1935

= Microsomacanthus m. López-Neyra, 1942 et Spassky-Spasskaïa, 1954.

Régions indienne (Indes) et paléarctique (Sakhaline).

Hôtes: Burhinus o. adicnemus (L.) [= Edicnemus scolopax (Gmel.)] aux Indes ; Calidris alpina sakhalina Vieillot et Calidris subminuta Middend. à Sakhaline (Krotov, 1952).

Types non consultables en dehors du pays d'origine.

Caractéristiques principales : Strobile : $2,3 \times 0,36 \mathrm{~mm}$. Crochets falciformes: 1.X.110-114 $\mu$. Ventouses glabres. Atrium GÉnItal inerme sans sacculus. Musculature non décrite. POCHE DU CIRRE très petite : $23 \times 13 \mu$. $\mathrm{PC} / \mathrm{AM}=1 / 10$. Euf : $52 \mu$. Reste de l'anatomie non précisé.

Remarque. - On peut se demander si la disposition si spéciale des glandes génitales (du type E) est conservée dans les anneaux mûrs normalement décontractés.

(9) Cf. cependant Hym. (H.) patersoni n. sp. dont le rostre est porteur de crochets également longs de $70 \mu$. 

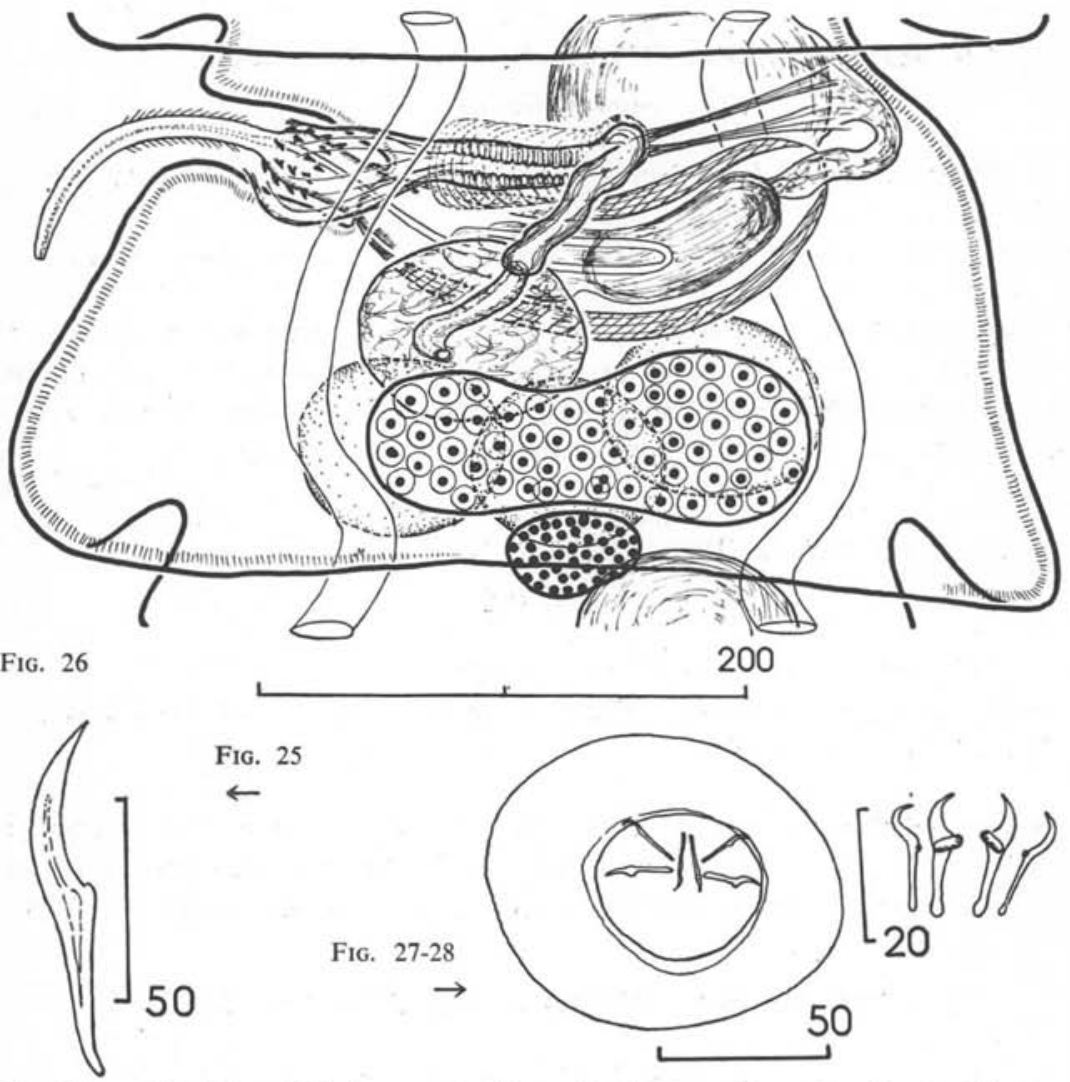

FIG. 27-28

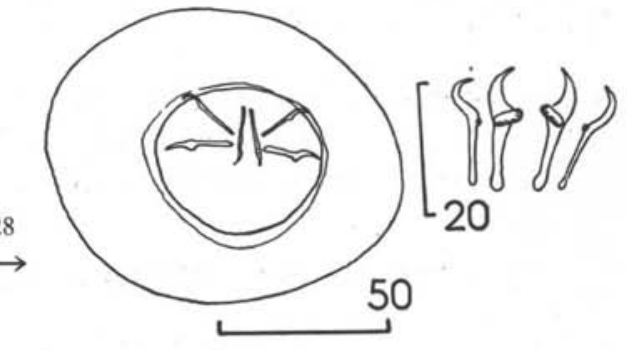

Fig. 25. $-H$. (H.) mudderbugtenensis (Baer, 1956). Type. Crocethia alba (Pall.). Crochets du rostre.

Fig. 26. - H. (H.) mudderbugtenensis. Anneau mûr. Vue ventrale. (Reconstitution partielle).

Fig. 27-28. - $H$. (H.) mudderbugtenensis. Cuf mûr et crochets de l'embryon.

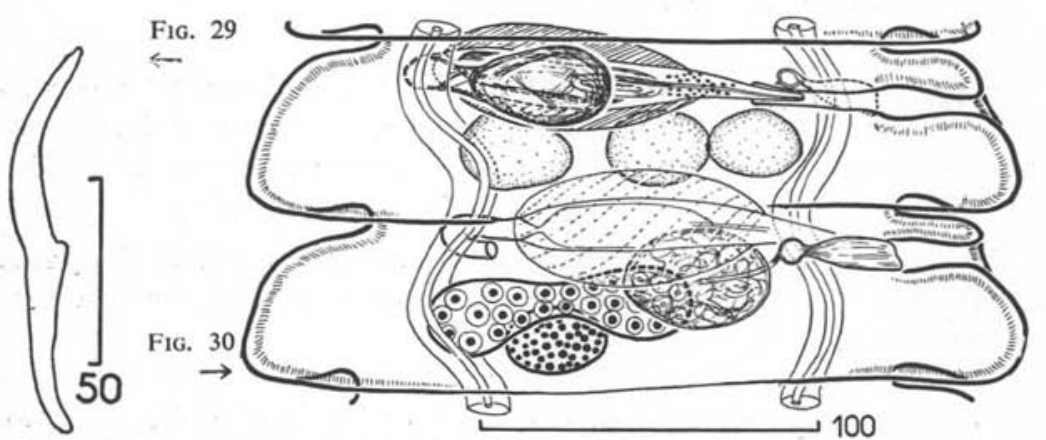

FIG. 29. - H. (H.) nitida (Krabbe). Type. Erolia maritima. Crochet du rostre.

Fig. 30. - H. (H.) nitida. Anneaux mûrs. Vue dorsale. Reconstitution partielle. Système mâle en haut et femelle en bas. 


\section{1) HYMENOLEPIS (HYM.) MUDDERBUGTENENSIS (Baer, 1956)}

nec Hym. nitida (Krabbe, 1869) sensu Baer, 1956.

Région néarctique (Groenland).

Hôte: Crocethia alba (Pallas).

Matériel observé : types ( $\mathrm{P}^{r}$ J. G. Baer, leg.).

Strobile : $50 \mathrm{~mm} \times 1 \mathrm{~mm}$. Subit un aplatissement inhabituel, mais naturel semble-t-il, dans le sens latéro-latéral, peut-être dû à la puissance des fibres rétractrices des conduits génitaux mâle et femelle. Nombre d'anneaux : 350 environ. CRochets falciformes : 1.X.84-86 $\mu$ (fig. 25). Ventouses glabres.

Musculature longitudinale interne de 14 fibres environ, de 20-22 $\mu$ de diamètre (et non pas 4 - Baer, 1956, p. 46) ; externe, d'une trentaine de fibres de $10 \mu$ de diamètre.

Atrium GÉNITAL glabre. Dépourvu de sacculus accessorius.

Poche DU CIRRE : $210-220 \mu \times 68-69 \mu$ de diamètre. Musculeuse. PC/AM $=1 / 2$.

CIRre : long de $150 \mu$ (cf. description de 1960, Ann. Par. hum, et comp., p. 565-566).

VAGIN : long de 220-230 $\mu$. Structure complexe :

$1^{\circ}$ La partie distale copulatrice se différencie en : $a$ ) une portion initiale de structure peu analysable, de $30 \mu$ de long; $b$ ) une portion moyenne se poursuivant à l'intérieur d'un manchon épais $(10 \mu)$ de fibres musculaires circulaires, sur une longueur de $70 \mu \times 25 \mu$ de diamètre externe. Sa paroi interne est tapissée de fines papilles juxtaposées (Baer, 1956). Ces deux portions vaginales sont maintenues parallèles au bord antérieur de l'anneau, souvent en avant de la poche du cirre, par de puissantes fibres rétractrices s'insérant le long du bord antiporal de l'anneau.

$2^{\circ}$ Le vagin tourne à angle droit en direction postérieure, sa paroi demeurant chitinoïde, mais dépourvue de papilles, sur une longueur de $40-45 \mu \times$ $13 \mu$ de diamètre.

$3^{\circ}$ La portion proximale non copulatrice, non chitinisée, est courte $(60 \mu)$, située dans le prolongement de la précédente, et se met en rapport avec le réceptacle séminal (fig. 26).

Disposition ovaire-Testicules de type D.

UTÉRUS gravide : nettement bilobé. CEUf mûr : coque externe : 70 et même $80-90 \mu \times 55 \mu$. Coque interne invisible. Embryon hexacanthe jusqu'à $45 \mu$ de diamètre. Crochets de l'embryon hétérogènes : crochets internes des paires latérales plus développés que les quatre autres crochets (fig. 27-28). 
Remarque. - La confusion qui a régné pendant cinquante années entre les deux espèces qui suivent, pourtant bien distinctes, nous incite à les redécrire l'une et l'autre d'une façon complète.

32) HYMENOLEPIS (HYM.) NITIDA (Krabbe, 1869)

nec Echinocotyle nitida Clerc, 1902-1903.

Syn. : Aucune des quatre synonymies proposées par Baer en 1956 ne semble devoir être retenue.

Région paléarctique (Iles Faroe). Hôte type: Tringa maritima Brünnich.

\section{Redescription de l'espèce :}

Matériel de description: types de Krabbe communiqués par M. le Professeur J.-G. Baer. Un cou et un scolex écrasés dans gomme-chloral avec les crochets. Le strobile correspondant, rétracté et décoloré, monté au baume. Coupes histologiques.

Strobile : longueur totale, $13 \mathrm{~mm}$; largeur maximale, $0,260 \mathrm{~mm}$.

Nombre de proglottis : 550 (90 anneaux de cou dans gomme-chloral + 460 anneaux dans le baume).

Le strobile entier doit comporter vraisemblablement une cinquantaine d'anneaux gravides supplémentaires et mesurer 2 à $2,5 \mathrm{~cm}$ environ à l'état rétracté et 3 à $3,5 \mathrm{~cm}$ au maximum.

PORES GÉNItAuX unilatéraux, dextres.

AtriUm GÉNItAl dépourvu de sacculus accessorius et d'épines.

Musculature: deux assises de faisceaux musculaires longitudinaux sur chaque face du Cestode, l'une externe formée de 30 à 40 fibres fines; l'autre plus interne formée de 10 à 13 fibres de grosse section.

CANAUX OSMO-RÉgulateURS : en position ventrale par rapport aux conduits génitaux sexuels. Diamètre du vaisseau ventral : 10-12 $\mu$; du dorsal : $2,5 \mu$. Il est impossible de voir s'il existe une anastomose transverse postérieure entre les canaux osmo-régulateurs ventraux.

Scolex (non observé par nous; cf. dessin original de Baer, 1956, $\mathrm{n}^{\circ} 56$, p. 44, d'après un cotype de Krabbe). Crochets : une couronne de 10 crochets à long manche dépourvu de garde. Taille moyenne : $108 \mu$ de long $(107,5-$ $110 \mu$ ). Longueur du manche, garde comprise : $53 \mu$; longueur de la lame : $55 \mu$. Epaisseur à la garde : $10 \mu$ (fig. 29). La segmentation du cou semble commencer immédiatement en arrière du scolex.

ANNEAUX: la composition du strobile peut se schématiser comme suit: 150 anneaux de cou; les organes mâles apparaissent avec netteté à partir du $170^{\circ}$ et les testicules persistent au moins jusqu'au $450^{\circ}$ anneau ; les organes femelles (sauf le vagin d'apparition plus précoce, simultanée aux organes mâles) n'apparaissent distinctement que vers le $450^{\circ}$ anneau. Au $550^{\circ}$, les œufs n'ont pas encore acquis leur maturité. 
Système génital mâle (fig. 30)

\section{Testicules:}

Régulièrement ovalaires, les trois testicules mesurent 23-25 $\times 13 \mu$; dans les anneaux jeunes $\left(170^{\circ}\right)$, de $150 \mu$ de large $\times 23 \mu$ de haut ; situés à la partie postérieure et dorsale du proglottis, leur position prédominante semble être en ligne, le testicule antiporal étant légèrement plus antérieur; le médian est le plus dorsal (type $\mathrm{C}$ ou $\mathrm{G}$ ).

[A ce stade, la poche du cirre mesure $45-50 \mu \times 10-11 \mu$, son enveloppe musculaire étant très mince $(1 / 2 \mu)$; elle est située entre les canaux osmorégulateurs droits et gauches, ne franchissant que les canaux osmo-régulateurs poraux].

Vers le $260^{\circ}$ anneau mesurant $175 \times 26 \mu$, les testicules ont acquis leur taille maximale $(37 \times 13 \mu-29 \times 20 \mu-24 \times 20 \mu)$.

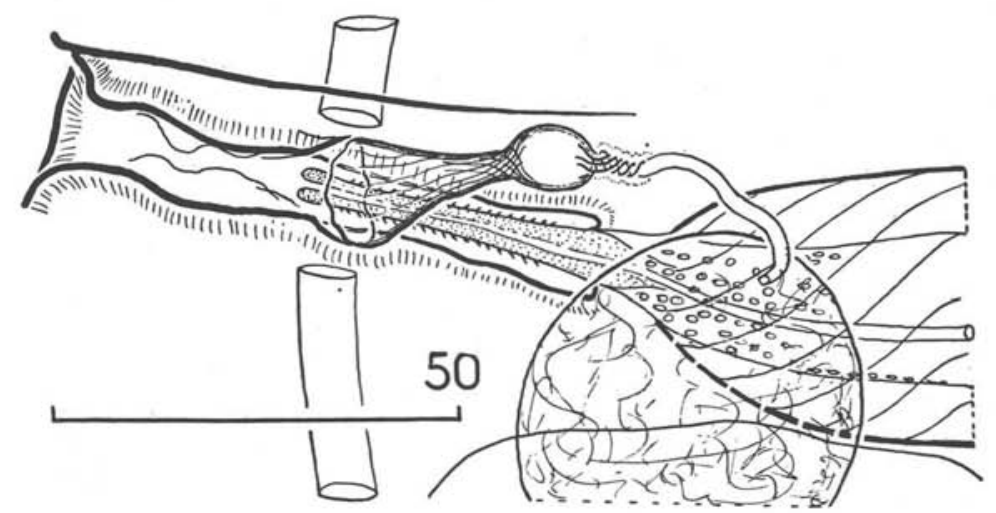

FIg. 31. $-H$. (H.) nitida. Conduits sexuels terminaux et atrium génital. Vue ventrale.

\section{POCHE DU CIRRE :}

A son maximum de développement (vers les $350^{\circ}-400^{\circ}$ anneaux, de $230 \times$ 26-30 $\mu$ environ), la poche du cirre mesure 70-77 $\mu \times 32 \mu$ de diamètre. De forme ovale allongée, elle est située parallèlement au bord antérieur de l'anneau et ne semble pas devoir dépasser les canaux osmo-régulateurs antiporaux. Parois formées de fibres musculaires longitudinales hélicoïdales très épaissies $(7,5 \mu)$, bien marquées.

Vésicule séminale interne tubulaire formant une mince boucle du côté poral, comme chez $H$. stellorae Deblock et coll., 1960, avant de se poursuivre par le canal éjaculateur. Il ne semble pas y avoir de différenciation chitinoïde du canal éjaculateur, comme chez H. mudderbugtenensis (Baer, 1956) ou H. charadrii Yamag., 1935, par exemple. 
Cirre court et mince, de 33-35 ×5 $\mu$, en forme de cône effilé, sans dilatation basale ; à l'état évaginé, il montre dans sa partie moyenne une spinulation fine et peu abondante sur une longueur de $20 \mu$ (épines de 0,5 à $1 \mu$ ), que l'on pourrait plutôt comparer à une ponctuation ; elle peut être trouvée invaginée.

3. Vésicule sÉminale externe. Située dorsalement par rapport à la poche du cirre, à son côté antiporal ; ovoïde ; $32 \times 19 \mu$ dans son plus complet développement. Le canal déférent qui unit les deux vésicules est bien figuré.

\section{Système génital femelle (fig. 30)}

Le vagin est le premier organe femelle différencié, dès le $170^{\circ}$ anneau; la naissance des autres organes femelles est nettement plus tardive.

OVAIRE : postérieur, ventral, médian, apparemment bilobé. Taille : 60-70 $\mu$ $\times 25 \mu$.

Vitellogène : postérieur à l'ovaire, ovoïde. Taille : $20 \times 13 \mu$.

RÉCEPTACLE SÉMINAL : très mal visible sur le strobile monté au baume. Les coupes histologiques le montrent ventral par rapport à la poche du cirre et situé sensiblement à son niveau. Ovoïde. Taille : $45 \times 20 \mu$ environ.

VAGIN : ventral par rapport à la poche du cirre. Sa morphologie s'apparente à celle de $H$. charadrit Yamaguti, 1935, in Deblock et coll., 1960 (fig. 31). Long de $70 \mu$ environ au total, il comporte trois parties distinctes: la première (proximale), de forme tubulaire, longue de $40-50 \mu$, fait suite au réceptacle séminal et court parallèlement à la poche du cirre; la deuxième est constituée par une dilatation sphéroïdale creuse, de 6 à $7 \mu$ de diamètre, jusqu'à 10 $15 \mu$ de long, à paroi d'apparence chitinoïde ; la troisième (distale), longue de 20 à $30 \mu$, en forme de cône évasé, met le vagin en rapport avec la paroi ventrale de l'atrium génital ; de part et d'autre de la dilatation, les parois chiti-

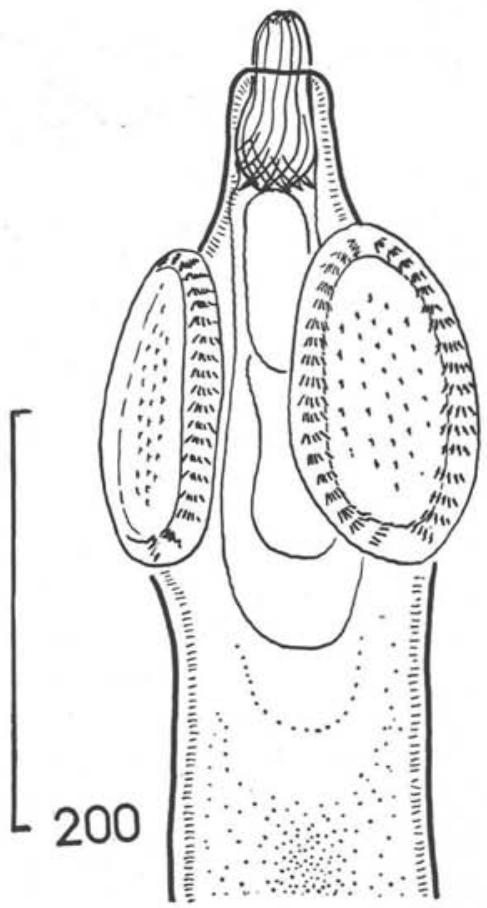

FIG. 32. -(E.) nitida (Clerc, 1902-1903). Scolex. noïdes vaginales apparaissent comme lignées longitudinalement. 


\section{ANNEAUX GRAVIDES :}

Utérus jeune sacciforme. Anneaux gravides à œufs mûrs inconnus. D’après Krabbe (fig. 135, pl. VI), les œufs sont sphériques et les crochets de l'embryon mesurent $13 \mu$ de long.

\section{Remarques :}

$1^{\circ}$ Cette étude faite, nous sont parvenus des Cestodes d'origine russe, dont la description se superpose exactement à celle des types (hôtes: Tringa maritima Brünn., Mer de Barents, M. M. Belopolskaïa determ. 1953 et leg.). Bien fixés et conservés, ils nous ont permis de lire rapidement ce qui était très difficilement analysable sur les types de Krabbe.
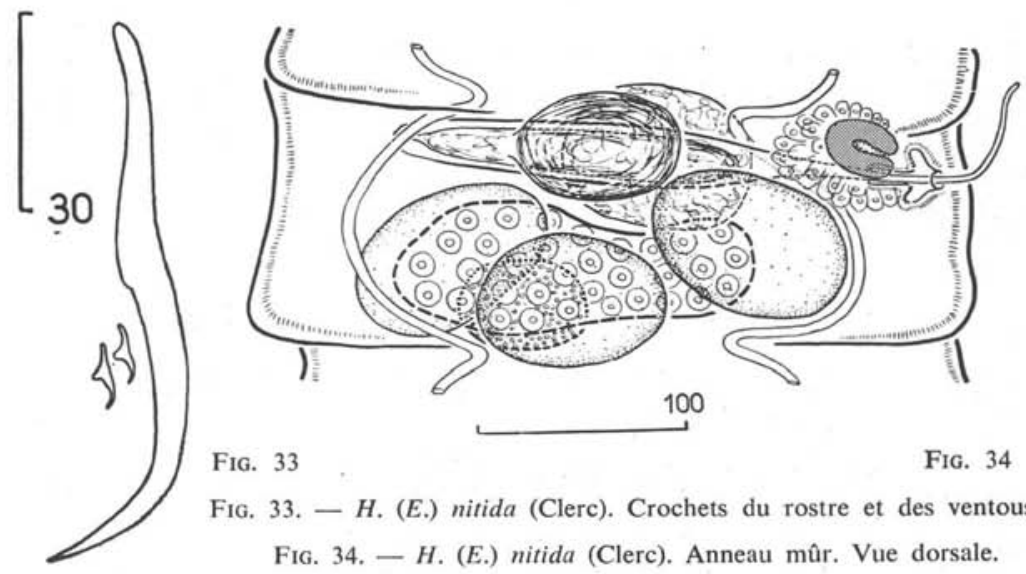

FIG. 33

Fig. 34

FIg. 33. - H. (E.) nitida (Clerc). Crochets du rostre et des ventouses.

Fig. 34. - H. (E.) nitida (Clerc). Anneau mûr. Vue dorsale.

Crochets de 110-111 $\mu$. Strobile de 385 anneaux, mesurant jusqu'à $25 \mathrm{~mm}$; poche du cirre : jusqu'à $120 \times 32 \mu$. Rapport $\mathrm{PC} / \mathrm{AM}>1 / 2$ dans l'anneau mâle, $=1 / 2$ dans l'anneau mûr et $<1 / 2$ dans l'anneau gravide. Testicules dans les anneaux mâles disposés pratiquement en ligne, le testicule médian très légèrement en arrière des deux autres. Ovaire : bilobé. Naît ventralement au testicule médian, parfois légèrement à gauche ou à droite de celui-ci. Eufs ovoïdes, membrane externe: $44-57 \times 30-44 \mu$; embryon hexacanthe: de $25 \times 25 \mu$ à $32 \times 25 \mu$; crochets de $13-15 \mu$, hétérogènes.

$2^{\circ}$ Nous avons également retrouvé cette espèce en deux exemplaires non gravides chez Erolia ruficollis minuta (Leisler), de la baie de Canche, en 1959. Strobile : $3 \mathrm{~mm} \times 0,2$; scolex : $200 \mu$ de diamètre ; ventouses : $130 \times 106 \mu$; 10 crochets falciformes de 113-120 $\mu$; anneau mûr: $212 \times 40 \mu$. Poche du cirre : $120-145 \times 22 \mu$. 


\section{3) HYMENOLEPIS (ECHINOCOTYLE) NITIDA Clerc, 1902-1903}

nec Echinocotyle nitida (Krabbe, 1869) sensu Clerc, 1902-1903 ; nec Hymenolepis nitidulans (Krabbe, 1882) sensu Baer, 1956.

Région paléarctique. Russie d'Europe (Oural, Clerc, 1902) et d'Asie: Sibérie orientale (Belopolskaïa, 1959) et occidentale (Dubinina, 1953). Chine (Shen Tseng, 1933).

Hôtes: Erolia minuta (Leisl.), E. ruficollis (Pall.), E. temninckii (Leisl.), Tringa stagnatilis (Bechst.), Limosa lapponica (L.). (Accidentellement chez Larus ichthyaetus Pall. et $L$. ridibundus L., Sibérie occidentale).

Matériel observé : une douzaine de strobiles d'origine russe dépourvus d'anneaux gravides, récoltés chez E. temninckii par Dubinina et chez E. ruficollis par Belopolskaïa, à qui nous devons leur communication.

Strobile : taille : $4 \mathrm{~mm}$ en moyenne $\times 0,36 \mathrm{~mm}$ de largeur maximale. Nombre d'anneaux : de 70 à 130 (sans anneaux gravides). Pores génitaux unilatéraux dextres. Musculature longitudinale interne formée de quatre faisceaux sur chaque face du Cestode. Canaux osmo-régulateurs poraux ventraux par rapport aux conduits sexuels et dépourvus d'anastomose transverse.

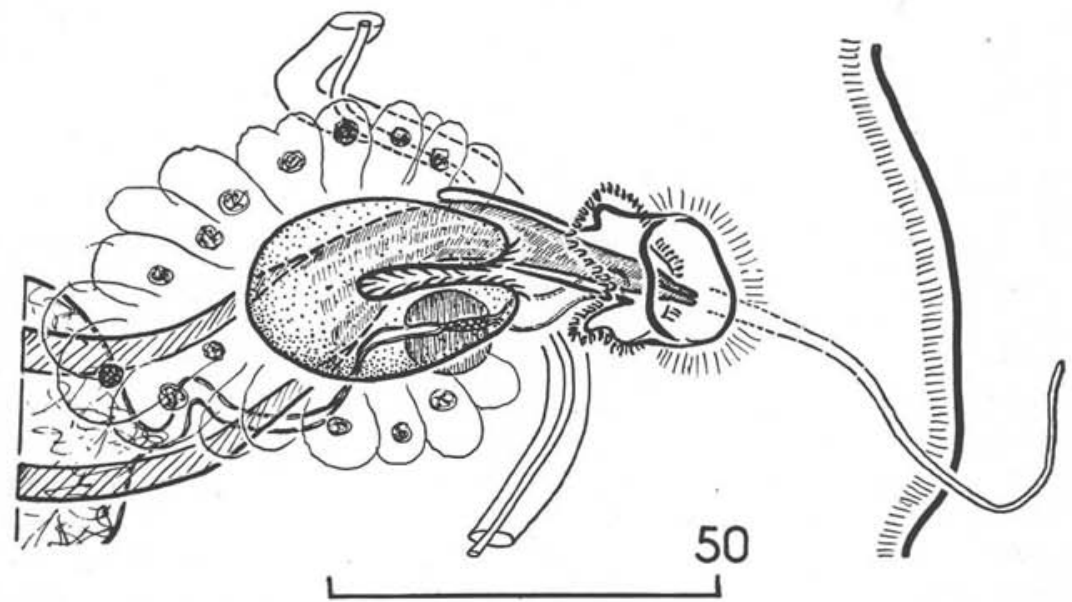

Fig. 35. - H. (E.) nitida (Clerc). Conduits sexuels terminaux et atrium génital. Vue ventrale. Sac accessoire dorsal, cirre (hachuré), médian, silhouette du cirre évaginé. Vagin ventral (en double trait).

Scolex : pyramidal à base rectangulaire par suite d'un léger aplatissement naturel dorso-ventral. Longueur : $200 \mu \times 150 \mu$ de largeur. Ventouse en ovale très allongé. Taille : $160 \times 80 \mu$. Leur bord musculeux de $18 \mu$ d'épaisseur est garni de 40 groupes de 5 crochets de 6-7 $\mu$ de long, disposés en éventail. Le 
fond des ventouses, nettement aplati et ne formant pas cupule comme chez ies Hymenolepis (Hymenolepis), est garni de cinq rangées longitudinales d'épines identiques aux précédentes (fig. 32).

Rostre : rétracté, mesure $170 \mu$ de long $\times 60 \mu$ de large. Dépasse le bord inférieur ventousaire. Sac du rostre: $260 \mu \times 60-80 \mu$; masse des crochets repliés dans le sac du rostre: $90 \times 28 \mu$. Crochets falciformes, 1.X.86-89 $\mu$ (fig. 33). Cou court: $100 \times 100 \mu$.

ANNEAUX : trapézoïdaux, non imbriqués les uns dans les autres. Taille maximale : $360 \times 80 \mu$ (fig. 34).

ATRIUM GÉNITAL: ampullaire dans les anneaux immatures et garni d'une couronne complète de crochets de 5-6 $\mu$ de long $\times 3 \mu$ de haut. Par la suite, l'atrium est plus déformable, mesure environ $26 \mu$ de diamètre $\times 13 \mu$ de profondeur et son armature paraît plus ou moins complète. Dorsalement par rapport à la base du cirre, se situe un sacculus accessorius musculeux, de $40-45 \times 20-25 \mu$. Sa lumière, longue de $25 \mu$, est garnie d'épines fines de $2,5 \mu$ et il est entouré d'un massif de cellules glandulaires bien différenciées de $80 \times 60 \mu$ de large (fig. 35).

\section{a) Système génital mâle}

- Testicules : au nombre de trois, disposés en triangle à sommet postérieur, parfois en ligne (types D ou G). Triangle généralement mieux marqué dans les anneaux jeunes que dans les anneaux mûrs où il a tendance à s'atténuer. Leur volume emplit la presque totalité du parenchyme médullaire des anneaux mâles. Taille: de $65 \times 40 \mu$ à $87 \times 70 \mu$, ou $90 \mu$ de diamètre.

- Poche DU CIRRE : en fuseau étroit très allongé, mesurant jusqu'à $190 \times$ $27 \mu$ (dans des anneaux de $340 \mu$ de large), s'étendant entre les canaux osmorégulateurs. Parois musculeuses épaisses de 3-4 $\mu$. Rapport $\mathrm{PC} / \mathrm{AM}>1 / 2$.

- Vésicule séminale INTERNE droite se poursuivant du côté poral par un canal déférent décrivant une boucle avant d'aborder le cirre.

- CIrre : à l'état d'évagination maximale, le cirre très long $(133 \mu)$, entièrement glabre (10), est filiforme $(7,5 \mu$ de diamètre à la base intra-atriale et 1 à $3,5 \mu$ de diamètre à l'extrémité).

- Vésicule séminale externe ovalaire dorsale par rapport à la poche du cirre, mesurant $90 \times 25 \mu$ de diamètre.

\section{b) Système génital femelle}

- OvaIre: prend naissance dans les anneaux mâles, sous le testicule médian. En ovale allongé transversalement, pourvu d'une faible bilobation. Taille : $50-55 \times 25 \mu$ jusqu'à $160 \times 75 \mu$ (dans les anneaux de $310 \mu$ de large).

(10) Clerc l'avait cependant décrit et figuré armé à la base de fins spicules.

AnN. de Parasitologie, T. XXXVII, No 5-6. - 1962. 
— Vitellogène postérieur à l'ovaire, ovoïde. Taille : de $25 \times 30 \mu$ à $50-80 \mu$.

- RÉceptacle séminal ovalaire, placé en avant du lobe poral de l'ovaire, ventralement par rapport à la poche du cirre. Taille : de $44 \times 25 \mu$ à $100 \times$ $50 \mu$.

- VAGIN ventral. Relativement peu apparent. Long de $80 \mu$ au total, hétérogène : 1) portion copulatrice tubulaire de $30 \mu$ de long $\times 5$ à $6 \mu$ de diamètre maximal à parois lisses peu chitinisées ; 2) il lui fait suite un rétrécissement spiralé légèrement chitinoïde, de $13 \mu \times 2 \mu$, entouré d'un muscle sphinctérien ; 3) portion proximale tubulaire de $40 \mu \times 2,5 \mu$ de diamètre, non chitinisée, en relation avec le réceptacle séminal. Absence de fibres rétractrices.

- AnNeaux mûrs : non observés.

Remarques. - Dans la systématique de Baer, où les divisions subgénériques ne sont pas admises, cette espèce doit changer de nom. Nous proposons Hymenolepis dubininae (Clerc, 1902-1903) nom. nov.

34) HYMENOLEPIS (HYMENOLEPIS) NITIDULANS (Krabbe, 1882) spec. inq.

$=$ Hymenolepis (Echinocotyle) n. (Kr., 1882) Fuhrmann, $1906 ;$ Echinocotyle $n$. (Krabbe, 1882) Fuhrmann, 1932 ; Hymenolepis $n$. Skriabine et Math., 1945 ; Nadejdolepis $n$. Spassky et Spasskaïa, 1954.

Région paléarctique (Europe).

Hôtes : Charadrius hiaticula L. et Erolia alpina (L.) in Krabbe ; Erolia alpina (L.), Grande-Bretagne (Southwell, 1924).

Caractéristiques (d'après Southwell, 1924): Strobile: $7 \times 0,14 \mathrm{~mm}$; CROCHETs : $1 . X .55$ (54 à $57 \mu$ d'après Krabbe). POCHE DU CIRRE musculeuse : 100 (?) $\times 25 \mu$ dans les anneaux mâles seuls décrits. PC/AM $>1 / 2$. CIRre glabre. VAGIN (?). Disposition ovaire-testicules de type G; atrium génital (?) (11).

Remarques: $1^{\circ} \mathrm{Ni}$ la description ni les figures de Southwell ne font état de ventouses ovalaires plates et spinulées ou de sac accessoire atrial. Il s'agirait donc plutôt d'un Cestode du sous-genre Hymenolepis. $2^{\circ} \mathrm{H}$. glareolae Singh, étant du sous-genre Echinocotyle, ne peut en être synonyme (cf. Baer, 1956, p. 50-51) car ses crochets font sans doute bien $65-72 \mu$ (cf. Hym. (Echino.) spec. inq., $\mathrm{n}^{\circ} 3$, p. 838). $3^{\circ}$ Baer, 1959 (p. 102) lui identifie un Cestode de Tringa glareola L. du Congo ex-belge. Cependant, si l'on reconstitue l'échelle

(11) Les erreurs d'interprétation qui figurent dans la légende de la figure 3, laissent supposer que le pore génital « très musculeux », correspond en fait à une mésinterprétation de l'image des parois de la poche du cirre en vue apicale. 
de la figure 63 par recoupement avec les données du texte, on évalue la longueur des crochets $(d)$ à $26 \mu$ de long environ, soit la moitié de la taille des crochets types; leur forme est différente (le rapport longueur de la lame/longueur du manche étant égal respectivement à $1 / 1,82$ et $1 / 1)$. Cette identification semble donc inacceptable.

Si une synonymie était à proposer pour l'espèce de Southwell, nous penserions plus volontiers à $H$. charadrii Yamag.

\title{
35) « HYMENOLEPIS 》 MEGALORHYNCHA (Krabbe, 1869)
}

Région néarctique (Groënland). Hôte : Erolia maritima (Brünn.).

Cette espèce redécrite par Baer, 1956 (p. 30-33, fig. 32-33) appartient en tait au genre Dilepis.

36) HYMENOLEPIS (HYM.) OWENI Moghe, 1933

\author{
= Drepanidotaenia o. Spassky et Spasskaïa, 1954; Echinocotyle o. Yamaguti, \\ 1959. \\ Région indienne (Indes). Hôte: Philomachus pugnax L.
}

Caractéristiques principales : STroBILE : $25-50 \times 1,2-1,5 \mathrm{~mm}$. Crochets claviformes: 1.X.50-58 $\mu$. Ventouses armées comme chez Echinocotyle (armature figurée à la périphérie seulement). ATRIUM ET MUSCULATURE non décrits. POCHE DU CIRRE très courte : $174 \times 53 \mu$ (200 $\mu$ d'après fig. 12). $\mathrm{PC} / \mathrm{AM}=1 / 15$ (d'après la fig. 11), 1/5 (d'après fig. 12) et $1 / 7$ environ d'après le texte. Cirre « en baguette», inerme. VAGIN tubulaire, long (300-400 $\mu$ au moins). Disposition ovaIRE-TEsticules du type $\mathrm{H} 2$ (ovaire postérieur). EUf : $80 \mu$.

37) HYMENOLEPIS (ECHINOCOTYLE) PARADOXUS (Saakova, 1958) nov. comb.

= Gonoscolex p. Saakova, 1958.

Région paléarctique (Roumanie, Delta du Danube).

Hôte : Limosa limosa (L.).

Matériel observé : quatre strobiles de $5 \mathrm{~mm} \times 0,27 \mathrm{~mm}$ de large, dépourvus d'anneaux gravides mûrs (M. M. Belopolskaïa, leg.).

Complément de description (fig. 36).

SColex : $310 \times 180 \mu$ de diamètre. Rostre long: $417 \mu \times 40 \mu$. Une couronne de 10 crochets falciformes de $83 \mu$ de long. Sac du rostre : $260 \times 78 \mu$. 
Ventouses très ovalaires, $160 \times 85 \mu$, à fond plat et à bord peu musculeux, armé de 40 rangées de 4 petits crochets identiques disposés en éventail, longs de $9-10 \times 2,5 \mu$ de haut. Le fond plat est garni de six rangées verticales de crochets identiques aux précédents.

MusculatURE ET CANAUX OSMO-RÉGULATEURS non observables.

Atrium GÉNital (fig. 37) :

1) Vaste et épineux: pourvu d'une couronne complète de deux ou trois rangées d'épines inégales disposées en quinconce n'entourant, semble-t-il, que l'orifice mâle de l'atrium.

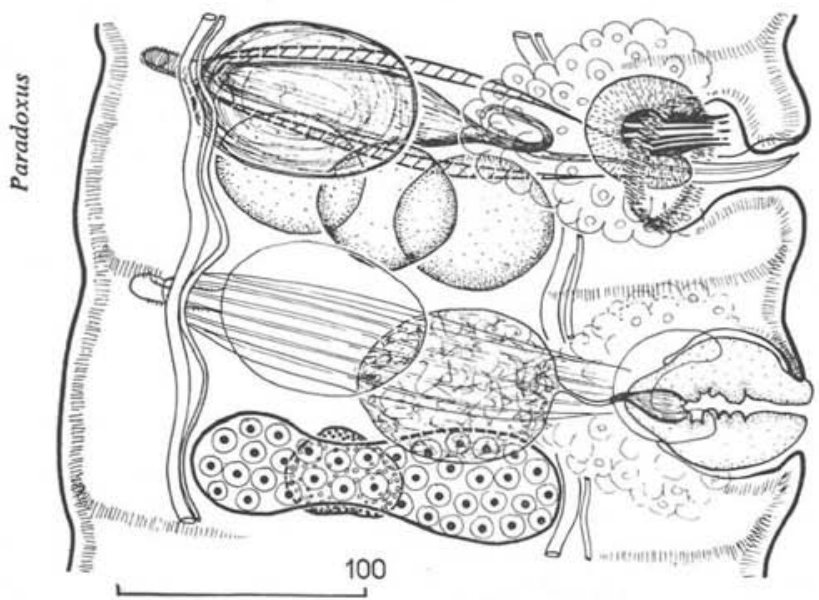

Fig. 36. $-H$. (E.) paradoxus (Saakova). Type. Anneaux mûrs. En haut, glandes femelles omises. En bas, glandes mâles omises; en pointillé, le capuchon vaginal. Vue dorsale. (Reconstitution semi-schématique).

2) Pourvu dorsalement d'un sacculus accessorius (= Gonoscolex) exceptionnellement volumineux, très puissamment armé, de taille et de forme variables, fonction de son état de rétraction-évagination. Tailles à son maximum de développement : a) rétracté : $35-40 \times 30-40 \mu$ de long ; cavité large de 12-15 $\mu$; paroi hyaline épaisse de $12-15 \mu ; b$ ) susceptible assez fréquemment de s'ouvrir comme une corolle de fleurs s'épanouit, les épines sacculaires ayant valeur d'étamines: au nombre de 10 , longues de $20-24 \mu$, régulièrement insérées longitudinalement contre la paroi interne par toute la longueur de leur manche. Le sac ouvert mesure $50 \times 50 \mu$ et sa cavité $40 \times 25 \mu ; c$ ) sur certains anneaux, le sacculus accessorius est capable de subir une énucléation partielle ou complète ne laissant adhérent au strobile qu'un panier musculaire dessinant son contour externe. 
POCHE DU CIRRE : toujours très longue, même dans les anneaux mûrs et gravides où elle atteint le bord antiporal de l'anneau. Taille : 180-200 $\times$ $30-40 \mu . \mathrm{PC} / \mathrm{AM}=1 / 1,3$.

CIRRE : totalement glabre, conique et relativement court à l'état évaginé : $65-70 \mu \times 12 \mu$ de diamètre à la base et $2 \mu$ à l'extrémité. Prolongeable par un stylet filiforme de $1,5 \mu$ de diamètre, long de 20-25 $\mu$.

VAGIN : long de $120 \mu$. Hétérogène : 1) deux lèvres très saillantes séparées par une fente dorsale envahissent l'hémisphère ventral de l'atrium génital et prolongent le vagin distal sur une longueur de $50 \mu$ environ $(=$ expansions de nature cuticulaire non musculeuses de Saakova) ; 2) portion copulatrice tubu-

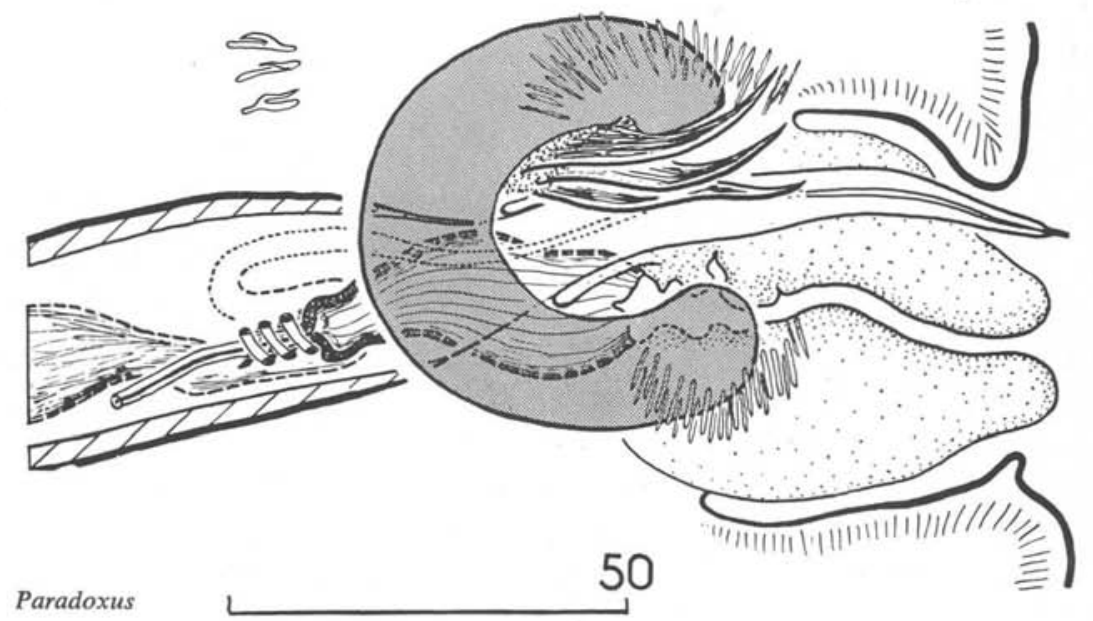

FIg. 37. - H. (E.) paradoxus (Saakova). Conduits sexuels terminaux et atrium génital. Vue dorsale. Sac accessoire dorsal (dont trois crochets seulement ont été figurés). Cirre glabre médian. Vagin proximal et distal et capuchon vaginal (en pointillé) ventraux. Crochets atriaux figurés dans une portion seulement du diamètre antéro-postérieur. A gauche détail de ceux-ci.

laire d'une cinquantaine de microns à parois faiblement chitinisées, occluse sur 10 microns de longueur par deux fibres musculaires hélicoïdales engrenées l'une dans l'autre ; 3) portion proximale tubulaire courte $(20 \mu)$ en rapport avec le réceptacle séminal. Disposition ovaire-Testicules de type non précisable (C, D ou G). Eufs inconnus.

38) HYMENOLEPIS (HYM.) PARANITIDULANS (Golikowa, 1959) nov. comb.

= Echinocotyle p. Golikowa, 1959 ; Nadejdolepis p. Spassky et Bobova, 1962. 
Région paléarctique: Russie d'Europe (Kœnigsberg) et d'Asie (Kamtchatka).

Hôte: Tringa alpina L. [égaré chez un Colymbiforme du Kamtchatka, Gavia stellata (Pontopp.)].

Caractéristiques principales : Strobile : 1,5-2 $\times 0,20 \mathrm{~mm}$. Crochets falciformes: 1.X.40-42 $\mu$. VEnTOUSES inermes. Musculature non précisée. Atrium génital dépourvu de sac accessoire. Poche DU CIRRE très musculeuse dépassant les C.O.R. antiporaux. PC/AM : 1/1,1. Cirre et vagin non décrits. Disposition ovaire-Testicules de type D (d'après le texte), bien que la figure montre les types B et $G$ ). Eurs mûrs inconnus.

Complément d'observation de Spassky et Bobova, 1962: Strobile : 5,5 $\times$ $1,2 \mathrm{~mm}$; scolex : $170 \mu$ de diamètre à rostre très allongé antérieurement. Poche du cirre : $90 \times 34 \mu$.

39) HYMENOLEPIS PORALE Meggitt, 1927 spec. inq.

= Sphenacanthus (?) p. López-Neyra, 1942 et Spassky-Spasskaïa, 1954.

Région paléarctique (Egypte). Hôte: Glareola pratincola (L.).

Ce STROBILE sans scolex, sommairement décrit et figuré d'après un anneau immature, constitue pour Baer, 1956 un nomen nudum. Néanmoins, Dubinina (1953) a trouvé un Cestode malheureusement dépourvu de scolex chez Glareola nordmanni Fisch.-W., dont la topographie des organes génitaux est analogue à celle décrite et figurée par Meggitt.

40) HYMENOLEPIS (HYM.) QUASIOWENI Dubinina, 1953

$=$ Echinocotyle $q$. Yamaguti, 1959.

Région paléarctique: Russie d'Asie (Sibérie occidentale).

Hôtes: Tringa glareola L., Tringla stagnatilis (Bechst.) et Philomachus pugnax L.

Caractéristiques principales : STRoвILE : $30-50 \times 1,5-3 \mathrm{~mm}$; scolex 240-275 $\mu$ de diamètre. Crochets claviformes 1.X.60-64. Ventouses d'Hymenolepis (Hym.), à fond garni d'épines $(6 \mu)$, mais à bord glabre. ATRIUM GÉNITAL dépourvu de sacculus. Musculature non décrite. Poche DU CIRRE courte. $\mathrm{PC} / \mathrm{AM}=1 / 6$ (d'après la figure). Cirre et vagin non décrits. Disposition OVAIRE-TESTICUles du type $\mathrm{H} 2$ (ovaire postérieur très allongé et aporal). Utérus sacculaire. EuFs inconnus.

41) HYMENOLEPIS (HYM.) RECTACANTHA Führmann, 1906, sp. inq.

$=$ Microsomacanthus (?) $r$. Spassky et Spasskaïa, 1954 .

Région paléarctique. Europe: Allemagne, Grande-Bretagne, Russie (Mer Blanche). 
Hôtes: Charadrius hiaticula L. (Fuhrmann); Haematopus ostralegus L. (Davies, Guinetsinskaiia et Naoumov).

Caractéristiques principales (d'après Davies, 1939): StrobILE : $35 \times$ $0,32 \mathrm{~mm}$. Scolex : $150-170 \mu$ de diamètre. Crochets claviformes 1.X.43 $\mu$. Ventouses inermes. Atrium inerme dépourvu de sacculus. Musculature non précisée. POCHE DU CIRRE non musculeuse longue : $70 \times 15 \mu$. PC/ AM $=1 / 2$. Cirre et vagin non précisés. Disposition ovaire-testicules des types B ou C. Eufs inconnus.

Remarque: Nous avons l'impression que Davies, trompé par son interprétation des crochets de Cambrensis, a redécrit $H$. rectacantha d'après des exemplaires de Cambrensis.

42) HYMENOLEPIS (HYM.) RECURVIROSTRAE (Krabbe, 1869) spec. inq.

= Dicranotaenia $r$. López-Neyra, 1942 ; Wardium $r$. Mayhew, 1925 et Spassky et Spasskaïa, 1954.

Région paléarctique : Afrique (Egypte) ; Oural (Clerc, 1910 in Dubinina); Sibérie occidentale (Dubinina).

Hôte: Recurvirostra avosetta L. (Krabbe, Dubinina, et peut-être Meggitt).

Caractéristiques du type : STROBILE : $1 \mathrm{~mm}$ de large, crochets chéliformes de 1.X.13-17 $\mu$. CIRre de $100 \times 8 \mu$. Eufs d'une quarantaine de microns de diamètre (d'après la fig. 188).

Récoltée en Egypte chez Recurvirostra avosetta L., l'espèce de Krabbe est ensuite sommairement décrite par Meggitt (1927) à l'occasion d'une nouvelle récolte de même provenance. On l'aurait entre-temps retrouvée en Suisse (Fuhrmann, 1926, p. 77). Des exemplaires d'un nouvel hôte capturé en Somalie, Himantopus himantopus L., sont rapportés par Joyeux, Baer et Martin, 1936 à une variété de l'espèce, magnosacco (nec longisacco Baer, 1959), en raison de la grande longueur de la poche du cirre ; cette variété est relevée au rang d'espèce par Joyeux et Baer en 1940 sous le nom d'H. tsengi, à la suite d'une comparaison avec de nouveaux exemplaires de Cestodes, assimilés à $H$. recurvirostrae et parasites de Capella macrodactyla (Bonap.) à Madagascar. Dubinina, en 1953, la signale en Sibérie occidentale chez l'hôte type et cite Clerc, 1910 dans sa bibliographie (Oural) sans ajouter de précision. Voge et Read (1954) l'auraient eux aussi retrouvée chez Recurvirostra americana en Californie (U.S.A.) et insistent sur la protérandrie extrêmement accusée des exemplaires américains. Enfin, Baer, en 1959, étudiant des Cestodes d'Himantopus himantopus (L.) congolais, publie une redescription partielle d'une espèce et une liste de synonymie dans laquelle figure $H$. tsengi, mais aussi 
H. himantopodis (Krabbe, 1869), H. recurvirostroides Meggitt, 1927 et Oligorchis kwangensis Southwell et Lake, 1939.

A notre avis, il est vraisemblable que sous le nom de $H$. recurvirostrae ont été étudiées, outre l'espèce type - bien mal connue $(12)-$, quatre espèces distinctes : celles de la Somalie, de Madagascar, du Congo et des Etats-Unis. Parmi les raisons qui suggèrent cette opinion, on peut citer : l'aspect particulier de l'œuf de l'Hymenolepis malgache, bien différent de celui figuré par Krabbe et s'apparentant à celui d'Haploparaxis sinensis Shen Tseng, 1932 par exemple ; le cirre épineux de $H$. tsengi et de l'espèce californienne, opposable au cirre court et glabre de l'Hymenolepis congolais et au cirre long de $100 \mu$ défini par Krabbe ; les différences considérables de taille: $1^{\circ}$ des crochets, variant du simple au double : 8-9 $\mu$ (Baer, 1959) et $13-17 \mu$ (Krabbe) ; $2^{\circ}$ des poches du cirre, égales depuis le $1 / 10^{\circ}$ de la largeur de l'anneau chez l'espèce malgache jusqu'aux 8 ou $9 / 10^{\circ}$ chez l'espèce congolaise (cf. fig. 64 Baer, 1959); $3^{\circ}$ des volumes des scolex d'après Meggitt : considérable pour recurvirostrae ( $500 \mu$ de diamètre) et réduit pour recurvirostroides $\left(130 \mu\right.$ de diamètre) $; 4^{\circ}$ la protérandrie exceptionnelle de l'Hymenolepis $s p$. de Voge et Read, son cirre épineux long d'une soixantaine de microns. En conséquence, nous pensons que $H$. recurvirostrae (Krabbe, 1869), H. tsengi Joyeux et Baer, 1940, H. recurvirostrae sensu Joyeux et Baer, 1959 constituent autant de $s p$. inquir., les seuls caractères ne prêtant pas à discussion étant, en fait, en dehors de ceux de Krabbe, les détails morphologiques assez précis fournis par Baer en 1959 concernant l'Hymenolepis congolais (dont nous serions tentés dès à présent de faire une espèce distincte, Hymenolepis $(H$.) edouardensis nom. nov.) et la description malheureusement incomplète des auteurs américains.

A l'appui de cette position dubitative, signalons encore qu'un autre Cestode possède des crochets identiques à l'espèce de Krabbe et des testicules en ligne - sans parler de $H$. hughesi Webster, 1947 - ; il s'agit d'un Oligorchis (O. paucitesticulatus Fuhrm., 1913) commun chez les Charadriiformes de la baie de Canche. Le nombre des testicules de cette espèce est susceptible, en valeur absolue, de varier de 1 à 10 ; mais, sur les quelque 200-250 anneaux sexués de nombreux strobiles, il ne se présente que trois ou quatre anneaux où ce chiffre soit différent de trois (quatre le plus souvent, très exceptionnellement deux ou cinq). Cet Hymenolepididae aberrant aurait pu abuser les auteurs n'ayant disposé que d'un matériel restreint, comme il nous a momentanément abusé nous-mêmes. La longueur de la poche du cirre y apparaît également très variable, dépassant plus ou moins largement les C.O.R. poraux.

Des recherches complémentaires précisant l'anatomie fine du recurvirostrae de Krabbe pourront seules clarifier ce problème, insoluble dans l'état actuel de nos connaissances.

(12) La taille de la poche du cirre donnée par Meggitt est manifestement erronée : pour un cestode large de $500 \mu$, elle mesure $125-160 \mu$ de long dans les anneaux mûrs, soit environ la moitié de ce qu'elle devrait avoir pour satisfaire son extension jusqu'aux C.O.R. antiporaux (à moins que cette disposition ne soit que transitoire). 
43) HYMENOLEPIS (HYM.) RECURVIROSTRAE sensu Joyeux et Baer, 1940 spec. inq.

nec $H$. recurvirostrae (Krabbe, 1869).

Région éthiopienne (Madagascar).

Hôte : Capella macrodactyla Bonap.

Caractéristiques principales : StrobiLE : $60 \times 1,5 \mathrm{~mm}$. CRochets chéliformes 1.X.13-15 $\mu$. Atrium non décrit. Musculature longitudinale interne : $2 \times 7-8$ faisceaux. POCHE DU CIRRE effilée : $120-180 \times 20-42 \mu$. PC/ AM $=$ 1/10. Cirre, vagin, ovaire non décrits. Testicules en ligne. Euf mûr: $48-51 \times 40-44 \mu$ avec coque interne renforcée et épaissie aux pôles $(6 \mu)$.

Remarque: - L'œuf caractéristique de cette espèce semble bien différent de celui figuré par Krabbe (pl. VII, fig. 188). Voir discussion de $H$. recurvirostrae $\left(n^{\circ} 42\right)$ dont seule une description nouvelle permettra de statuer sur le sort de ses espèces satellites.

44) HYMENOLEPIS (HYM.) RECURVIROSTROIDES Meggitt, 1927 spec. inq. ou nomen nudum

= Dicranotaenia $r$. López-Neyra, 1942 ; Wardium $r$. Spassky et Spasskaïa, 1954. Syn. : H. recurvirostrae (Krabbe, 1869), selon Baer, 1959.

Région paléarctique : Afrique (Egypte).

Hôte: Gallinago sp.

Caractéristiques : $30 \mathrm{~mm} \times 0,8$. CROCHETs chéliformes: 1.X.10-15 $\mu$. PoCHE DU CIRRE : courte, $96-110 \times 17 \mu$. PC/AM $=1 / 8$. Testicules en ligne. Reste de l'anatomie inconnu. Pas d'iconographie.

Remarque. - Pourrait se rapporter à Oligorchis paucitesticulatus Fuhrm., 1913.

45) HYMENOLEPIS (HYM.) SKRIABINISSIMA (Krotov, 1952)

$=$ Dicranotaenia $s$. Krotov, 1952.

Région paléarctique (Sakhaline).

Hôte : Calidris subminuta (Middendorff).

Caractéristiques principales : STRoBile : $25 \times 1,18 \mathrm{~mm}$. SCOLEX : $184 \mu$ de diamètre à rostre court et large $(100 \mu)$. CROCHETs chéliformes, au nombre de $68-70$, de $9-12 \mu$. Ventouses inermes de $50 \mu$ de diamètre. Musculature et atrium génital non décrits. POCHE DU CIRRE : $147 \times 55 \mu$. PC/ AM $=1 / 4$ 
à 1/5. Cirre et vagin non décrits. Disposition ovatre-testicules du type D (figurée du type G). Utérus sacculaire. GEuFs mûrs inconnus.

46) HYMENOLEPIS SPHAEROPHORA (Rud. 1810) Fuhrm., 1906 nomen nudum

Les crochets et l'anatomie de ce Cestode nous sont inconnus; on ignor même s'il s'agit d'un Hymenolepis (cf. Fuhrmann, 1926, p. 78).

47) HYMENOLEPIS (HYM.) SPINOSA Von Linstow, 1906

= Dicranotaenia s. López-Neyra, 1942 ; Kowalewskius $s$. Yamaguti, 1959.

Probablement synonyme de l'espèce suivante.

Région indienne (Ceylan).

Hôte : Rostratula capensis L.

Caractéristiques principales : Strobile : $15 \times 0,6 \mathrm{~mm}$. SCOLEX : $220 \mu$ de diamètre. Crochets claviformes: $1 . X .28 \mu$. Atrium génital inerme dépourvu de sacculus. Musculature interne : $2 \times 4$ faisceaux longitudinaux. PoCHE DU CIRRE volumineuse $(200 \mu$ ?). PC/AM $=1 / 2$ environ. VAGIN non étudié. Testicules : un antérieur et deux postérieurs. GEvfs : $47 \mu$.

Remarque. - Le cirre, décrit comme épineux, a été figuré glabre.

48) HYMENOLEPIS (HYM.) STYLOIDES Fuhrm., 1906

$=$ Weinlandia s. Shen Tseng, 1933 ; Dicranotaenia $s$. Skriabine et Mathevossian, 1945 ; Microsomacanthus s. Spassky et Spasskaïa, 1954.

Synonyme de Hym. (H.) spinosa von Linstow, 1906, selon une hypothèse de Spassky et Spasskaïa, 1954.

Région paléarctique : Afrique (Egypte).

Hôte: Vanellus aegypticus (L.).

Caractéristiques principales : Strobile: $20 \times 0,57 \mathrm{~mm}$. CROCHETs claviformes: 1. X.30 $\mu$. Atrium génital dépourvu de sacculus et d'épines. Musculature longitudinale de $2 \times 4$ faisceaux. CirRe non décrit. Vagin tubulaire étroit. Disposition ovaIRE-Testicules de type C. Utérus sacciforme. EUfs : $48 \mu$ de diamètre.

Remarque. $-1^{\circ} \mathrm{Ni}$ la coquille typographique du texte de Führmann ni les figures ne permettent de connaître approximativement la taille de la poche du cirre. Néanmoins, la valeur de 216 microns semble raisonnable, étant donné la position du testicule antiporal. La description de $H$. $(H$.) spinosa (où $2 / 3$ 
d'anneau correspondent à $200 \mu$ ) ne s'éloigne donc pas de celle de $H$. $(H$.) styloides. $2^{\circ}$ Shen Tseng (1933) rapporte à cette espèce un Hymenolepis de Rhynchea capensis autopsié en Chine, sans apporter de précisions intéressantes.

\section{9) HYMENOLEPIS (ECHINOCOTYLE) TENUIS (Clerc, 1906)}

\section{$=$ Echinocotyle $t$. Clerc, 1906.}

Régions paléarctiques: Russie d'Europe (Oural, Mer de Barentz) et d'Asie (Sibérie occidentale).

Hôtes: Totanus sp. (Clerc) ; Philomachus pugnax (L.), Erolia temninckii (Leisler), Phalaropus lobatus (L.) Dubinina, 1953 ; P. lobatus (L.) Belopolskaïa, 1959.

Caractéristiques principales: Strobile : $35 \times 0,5$. Scolex : $140 \mu$ de diamètre. Crochets claviformes: 1.X.20 (20-23 $\mu$ pour Dubinina). Ventouses typiques d'Echinocotyle, épineuses, longues de $130 \mu$. Musculature interne du strobile: $2 \times 4$ bandes longitudinales. Structures atriales non précisées. POCHE DU CIRRE courte: 100 à $150 \mu$ (ne dépassant pas les C.O.R. poraux pour Dubinina, fig. 11). $\mathrm{PC} / \mathrm{AM}=1 / 6,6$. CIRre court et armé (Dubinina). Disposition ovaire-TESTICUles du type B ou C. Eufs inconnus.

Remarque. - La diagnose de Meggitt, 1927 [Erolia alpina (L.), Egypte] doit être erronée si l'on en croit la redescription de Dubinina; le Cestode égyptien est en effet pourvu d'une poche du cirre beaucoup trop longue, atteignant l'axe longitudinal du strobile.

50) HYMENOLEPIS (HYM.) TSENGI Joyeux et Baer, 1940, sp. inq.

= Dicranotaenia $t$. López-Neyra, 1942 $;=$ Wardium $t$. Spassky et Spasskaïa, 1954.

nec $H$. recurvirostrae (Krabbe, 1869) sensu Baer, 1959.

nec $H$. recurvirostrae magnosacco, Joyeux et coll., 1936.

Région éthiopienne (Somalie Nord).

Hôte: Himantopus himantopus (L.).

Caractéristiques principales : TAILlE : $80 \times 1 \mathrm{~mm}$; CROCHETS chéliformes : $1 . X .11 \mu$ (garde, lame et manche de même longueur). Atrium génital non décrit. Musculature longitudinale interne de 8-10 faisceaux. Poche DU CIRRE : $350-400 \mu$ (jusqu'à $550 \mu$ ). PC/ $\mathrm{AM}=1 / 1,5$ environ. CIRre finement armé à sa partie distale. VAGIN non décrit. Disposition ovaIRE-TESTICUles de type G ou D. Eurs sans épaississement polaire.

Cf. discussion de $H$. recurvirostrae (Krabbe, 1869) et de l'espèce suivante (43). 
51) HYMENOLEPIS (HYMENOLEPIS) ULIGINOSA (Krabbe, 1882)

\author{
Fuhrm., 1906, sp. inq.
}

Région paléarctique (Europe) ; (Egypte ?).

Hôtes types : Numenius phaeopus (L.) ; Machetes pugnax (L.) ; Lymnocryptes gallinula (L.), signalés in Joy. et Baer, 1936 ; Otis houbara Desf. Meggitt, 1927 - problématique.

Caractéristiques principales: Strobile : $4 \mathrm{~mm} \times 0,25 \mathrm{~mm}$. Crochets chéliformes: $1 . X .42-45 \mu$ (lame $>$ garde). CirRe cylindrique de $40 \times 4 \mu$ glabre (?). Reste de l'anatomie inconnu.

\title{
52) HYMENOLEPIS (ECHINOCOTYLE) URALENSIS Clerc, 1902-1903
}

$=$ Echinocotyle u. Clerc, 1902 ; Fuhrmaniella u. Shen Tseng, 1933.

Syn.: Hym. (Echin.) hypoleuci (Singh, 1952) [Baer, 1956, p. 80].

Région paléarctique : Russie d'Europe (Clerc; Skriabine et Mathevossian, 1945) et d'Asie (Sibérie occidentale, Dubinina, 1953).

Hôtes: Actitis (= Tringa) hypoleucos (L.) ; Arenaria interpres (L.) ; Tringa totanus (L.) ; Tringa glareola (L.) ; T. nebularia (Gunn.) ; T. stagnatilis (Bechst.).

Caractéristiques principales: Strobile: $30-42 \times 1,2 \mathrm{~mm}$. Scolex: $290 \mu$ de diamètre environ; crochets claviformes: 1.X.64-66 $\mu$. Ventouses typiques d'Echinocotyle, ovales, armées (bords et fond), $220 \times 130 \mu$ environ (Dubinina 1953, fig. 12). Musculature longitudinale: $2 \times 4$ faisceaux longitudinaux. Atrium géNITAL armé, pourvu d'un sacculus. POCHE DU CIRRE petite. $\mathrm{PC} / \mathrm{AM}=1 / 3,5$ environ. Disposition ovaire-Testicules de type $\mathrm{D}$. Reste de l'anatomie imprécisé.

Remarque. - L'espèce de Southwell, 1920 (in Gallinago sp., Assam) possède des crochets beaucoup trop longs $(74 \mu)$ pour se rapporter à l'espèce de Clerc. Fort douteuse également est la diagnose de Shen Tseng 1933 (in Tringa americana, Chine), basée uniquement sur la forme des crochets, les ventouses rondes de $84 \mu$ de diamètre étant fort différentes de celles de la redescription de Dubinina, 1953.

\section{3) HYMENOLEPIS (HYM.) VAGINATA Baczynska, 1914}

= Sphenacanthus $v$. López-Neyra, 1942 et Spassky-Spasskaïa, 1954.

Région paléarctique: Afrique (Egypte).

Hôte : Recurvirostra avosetta L.

Strobile : $40 \times 1,13 \mathrm{~mm}$. Scolex : $195 \mu$ de diamètre. Crochets claviformes : $1 . X .36 \times 10 \mu$ de haut (à garde très épaissie). Ventouses circulaires gla- 
bres. Musculature interne de 12 à 14 faisceaux. Atrium génital dépourvu de sac accessoire et d'épines. Disposition ovaire-testicules de type B ou C. PoChE DU CIRRE : $260 \mu$ de long ; PC/ AM $=1 / 3,5$. CirRe finement épineux. VAGIN en entonnoir $(180 \mu$ de long ?), très musculeux, à bandes radiaires. UTÉRUs sacciforme. GEufs mûrs inconnus.

\section{4) HYMENOLEPIS VALLEI (Stossich 1892), nomen nudum}

Cette espèce, dont on ne connaît rien de la morphologie ni des mensurations, peut être définitivement considérée avec Baer, 1956 comme nomen nudum.

\section{C) DESCRIPTION D'ESPECES NOUVELLES}

\section{1) HYMENOLEPIS (HYM.) BELOPOLSKAIAE n. sp.}

Hôte : Crocethia alba (Pall.).

Date de récolte : août 1945.

Localisation géographique: Russie d'Asie (Sibérie occidentale : mer du Japon).

Matériel de description: deux strobiles décontractés, dont un seul mûr, mais dépourvu d'anneaux gravides (M. M. Belopolskaïa, leg.).

\section{Description de l'espèce.}

TAILlE : longueur $17 \mathrm{~mm} \times 0,45 \mathrm{~mm}$.

Scolex : typique du sous-genre Hymenolepis. Diamètre transverse : $235 \mu \times$ $310 \mu$ de long. Ventouses : $110 \mu \times 90 \mu$. Bord musculaire : 20-25 $\mu$. Dépourvues d'épines cuticulaires. Rostre invaginé : $350 \mu$ de long (jusqu'au fond du sac) $\times 83$ u dépassant largement le niveau du bord postérieur des ventouses (fig. 38).

Crochets : une couronne de 10 crochets falciformes sans garde et à long manche, de $85-87 \mu$ (fig. 39).

Cou : $450 \times 150 \mu$.

Strobile : formé d'au moins 200 anneaux plus larges que hauts, non imbriqués. On compte: 100 anneaux immatures, 70 anneaux mâles environ, 30 anneaux mûrs.

Musculature INTERNE: difficile à dénombrer, mais formée de plus de quatre faisceaux longitudinaux par face.

CANAUX EXCRÉTEURS : deux paires de canaux osmo-régulateurs longitudinaux non anastomosés. Franchissent ventralement les conduits génitaux.

ATRIUM GÉNITAL: profond, unilatéral, dextre, inerme et dépourvu de sacculus. 


\section{1) Anneaux mâles (fig. 40)}

Trapézoïdaux. Rapport longueur/largeur $=1 / 2$. Taille moyenne : $156 \times$ $365 \mu$. Les trois testicules se situent dorsalement en triangle ouvert antérieurement (type D). Les massifs embryonnaires des glandes femelles naissent ventralement dans l'axe du strobile sous le testicule médian.

La POCHE DU CIRRE peu musculeuse atteint les canaux osmo-régulateurs antiporaux en franchissant l'axe longitudinal du strobile. Taille : $210 \times 32 \mu$. Rapport poche du cirre/largeur de l'anneau $=1 / 1,8$.

CIRre : à l'état invaginé, des épines sont bien visibles sur une longueur de $115 \mu$ à l'intérieur de la poche. A l'état évaginé, le cirre mesure $93 \mu \times 18 \mu$ de diamètre maximal et n'est pas sans évoquer le cirre de $H$. amphitricha (Krabbe) : cylindrique et fortement épineux, sauf à ses extrémités; les plus fortes épines mesurent 5-6 $\mu$ de long $\times 3 \mu$ de large $\times 2,5 \mu$ d'épaisseur. Il est parcouru dans son axe par le déférent qui se poursuit dans la poche par un tube chitinoïde qui la parcourt sur toute sa longueur (fig. 42).
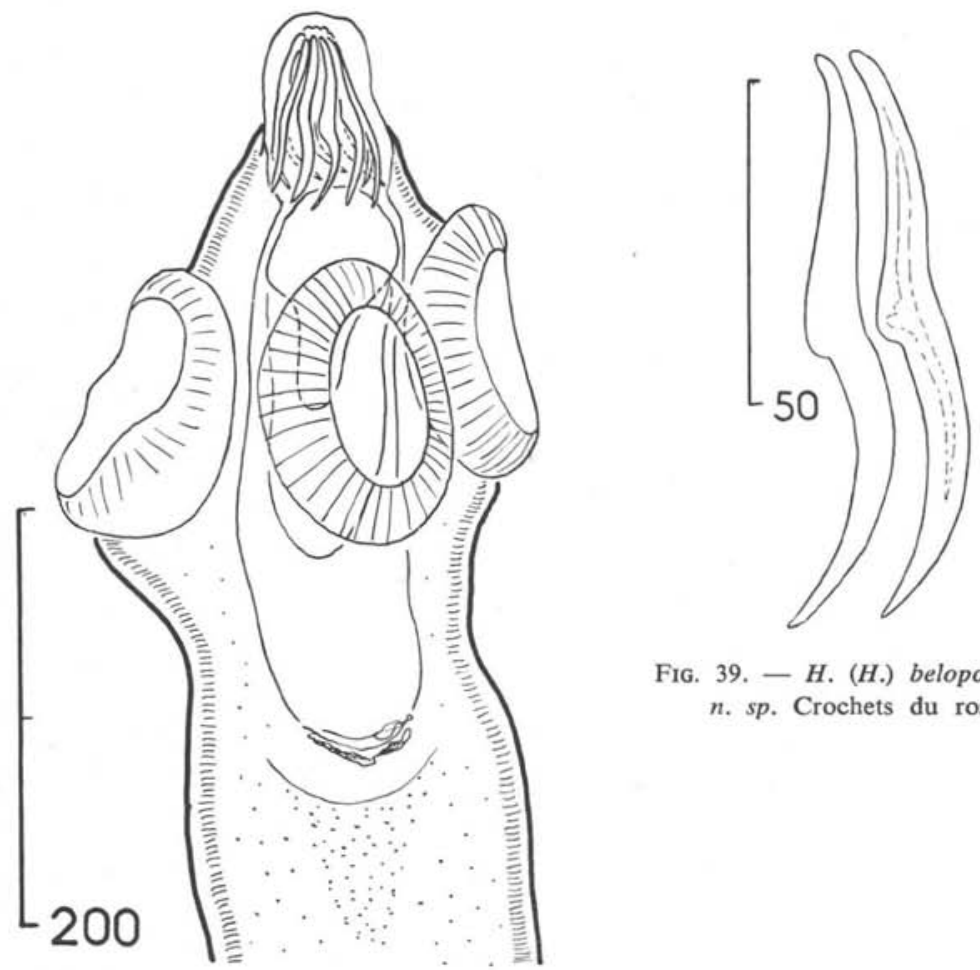

Fig. 39. $-H$. $(H$.) belopolskaiae n. $s p$. Crochets du rostre.

FIG. 38. - H. (H.) belopolskaiae n. sp. Scolex. 


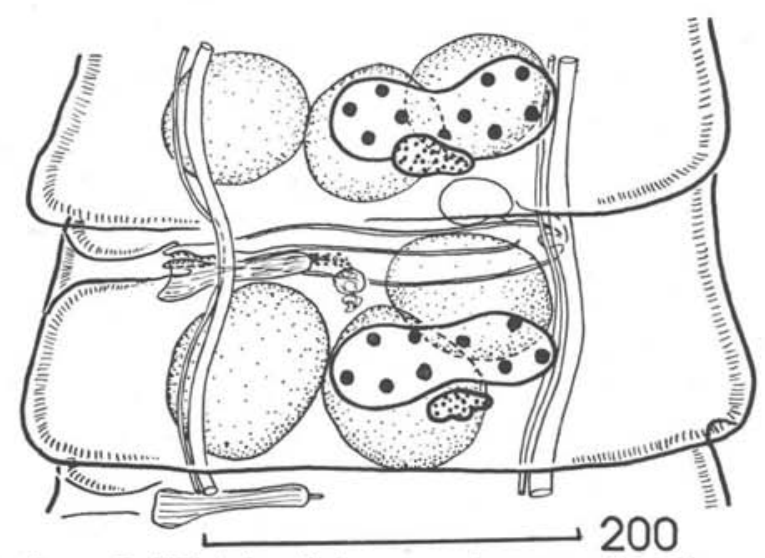

Fig. 40. $-H$. $(H$.$) belopolskaiae n. sp. Anneaux mâles. Vue ventrale.$

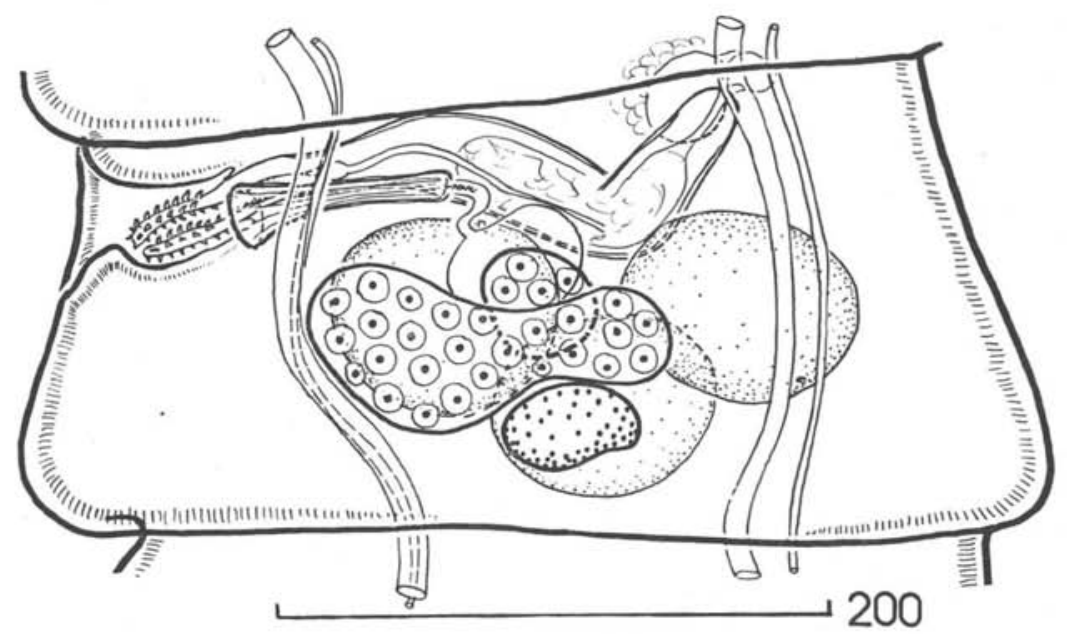

Fig. 41. $-H$. $(H$.$) belopolskaiae n. sp. Anneaux mûrs. Vue ventrale.$

VÉSICUle SÉminAle EXTERNE : dorsale, située au niveau de l'extrémité antiporale de la poche du cirre. Non observée à l'état de réplétion.

\section{2) Anneaux mûrs (fig. 41)}

TAIlle : $390 \times 200 \mu$. OvaIRE : bi- ou trilobé, médian, ventral, $130 \times$ $65-80 \mu$. VITELlogène : ovoïde, postérieur à l'ovaire, $26 \times 53 \mu$. RÉcEPTACLE SÉmINAL ovoïde, ventral, sub-médian, situé entre ovaire, testicule poral et poche du cirre. Non observé à l'état de réplétion. 


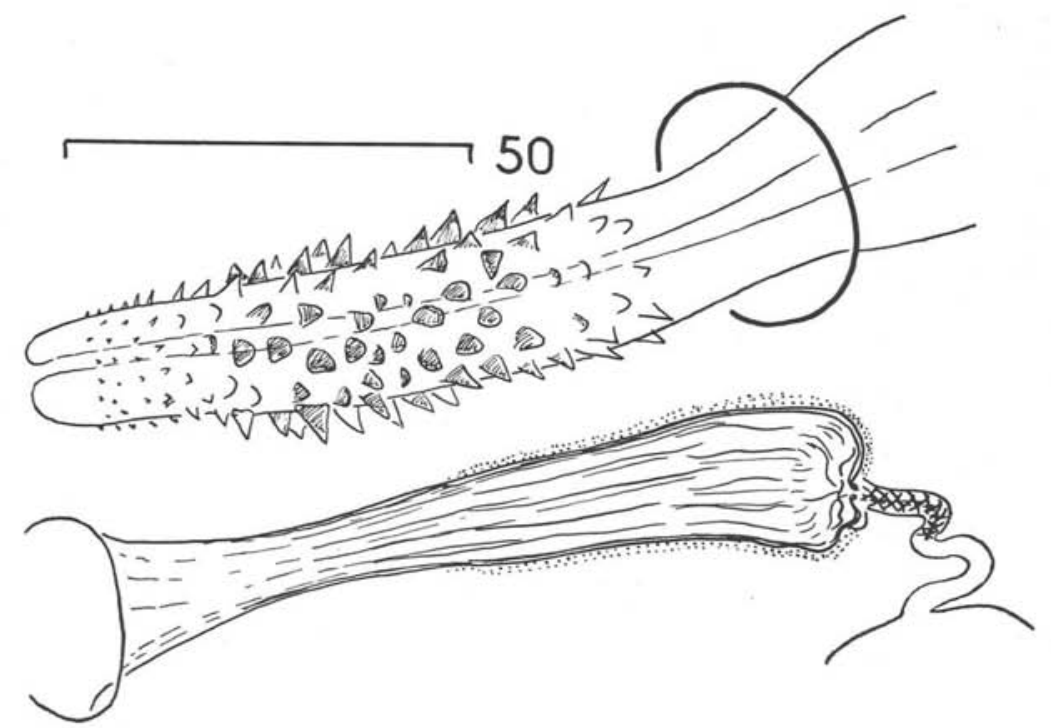

FIG. 42. $-H$. $(H$.$) belopolskaiae n. sp. Cirre évaginé et vagin.$

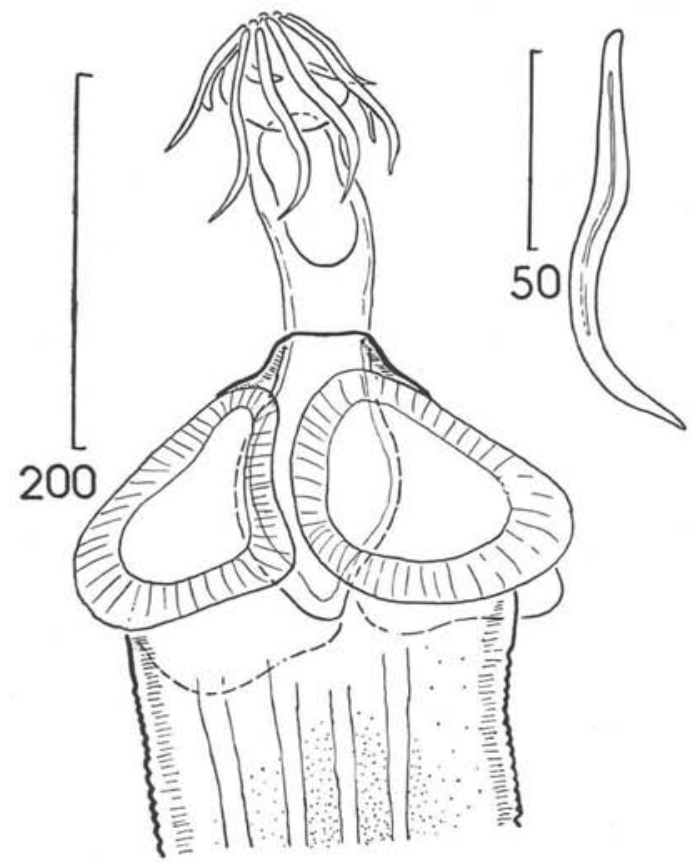

Fig. 43. - H. (H.) capetownensis n. sp. Scolex et crochet du rostre. 
VAGIN ventral par rapport à la poche du cirre et légèrement en arrière. Différent d'aspect de celui d' $H$. amphitricha. Longueur totale : $110 \mu-130 \mu$ environ. Sa portion porale initiale est chitinoïde, finement lignée longitudinalement par suite de la présence de nombreux plis, cylindrique, large et rectiligne, sur une longueur de $80-120 \mu \times 20 \mu$ de diamètre; puis l'organe se rétrécit brusquement pour former un fin canal de $30 \mu$ de long dont les épaississements chitinoïdes subissent une torsion sur eux-mêmes sur la moitié de son parcours, avant de disparaître pour laisser place au vagin membraneux qui aborde le réceptacle séminal. Il ne semble pas y avoir de sphincter ni de fibres rétractrices (fig. 42).

La POCHE DU CIRRE dans les anneaux mûrs est plus ou moins sinueuse et mesure $200 \mu \times 35 \mu$ de diamètre. Rapport : longueur de la poche du cirre/ largeur de l'anneau $=1 / 1,8$. Elle demeure peu musculeuse. Les testicules mesurent $80 \mu$ de diamètre.

\section{Discussion.}

Les deux seules espèces d'Hymenolepis de Charadrii pourvues d'un cirre d'aspect analogue à celui de l'espèce décrite sont $H$. amphitricha (Rud.) et $H$. hamasigi Yamag. ; comme toutes deux possèdent des crochets chéliformes, nous considérons l'espèce décrite comme nouvelle et sommes heureux de la dédier à Mme M. M. Belopolskaia, de l'Université de Léningrad, qui nous l'a communiquée.

\section{2) HYMENOLEPIS CAPETOWNENSIS n. sp. nec H. nitida (Krabbe) sensu Baer 1956}

Hôte: Crocethia alba (Pall.).

Date de récolte : novembre 1955. (N.F. Paterson leg. Baer).

Localisation géographique: Afrique du Sud (Capetown).

\section{Description de l'espèce.}

Matériel de description : sept exemplaires complets en bon état de fixation mais très rétractés (sauf un), colorés au carmin et montés au baume dans la même préparation qu'Hymenolepis patersoni $n$. $s p$.

Strobile : longueur totale 4 à $5 \mathrm{~mm}$ à l'état rétracté. Largeur maximale : 340 à $440 \mu$ quelques anneaux avant la fin de la chaîne, les tout derniers gravides étant plus étroits. Nombre de proglottis : 150 à $190 ; 70$ à 90 anneaux de cou, 40-60 anneaux sexuellement mûrs, 40-50 anneaux gravides, 3-4 anneaux à œufs mûrs. Pores génitaux unilatéraux, dextres. Atrium GÉNITAL inerme pourvu antérieurement d'un sacculus accessorius du type \& peigne atrial », de 15 à $16 \mu$ de long $\times 13 \mu$ de hauteur ; l'arc interne est garni de 


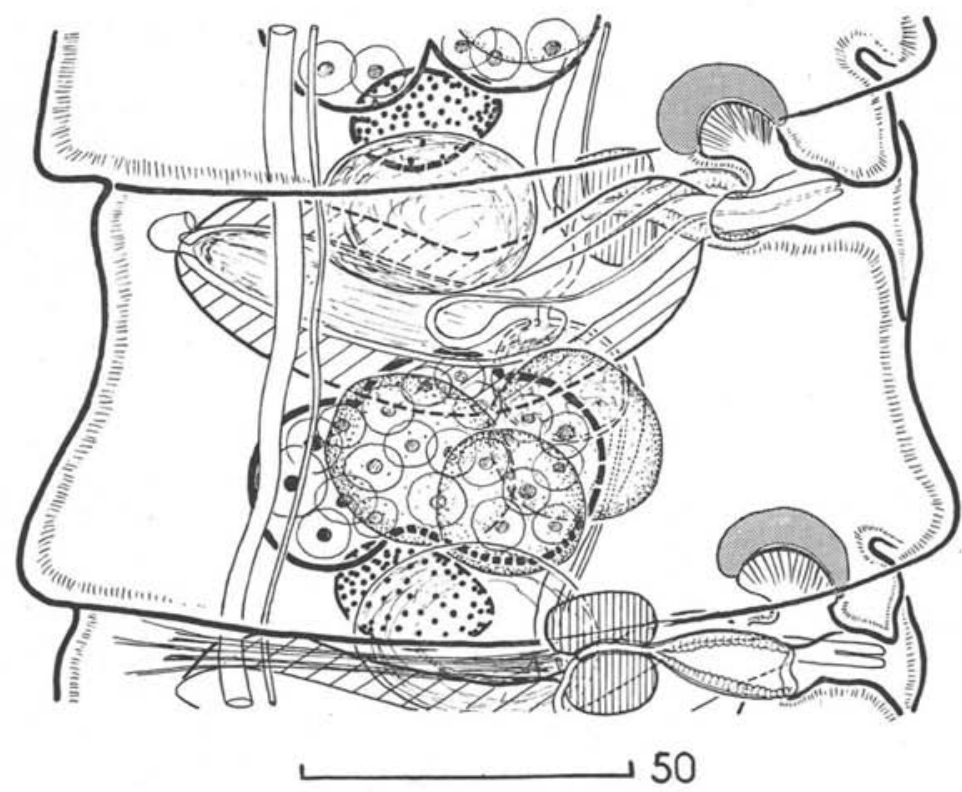

Fig. 44. - H. (H.) capetownensis n. sp. Anneau mûr. A la partie inférieure, mise en évidence des structures vaginales. Vue dorsale.

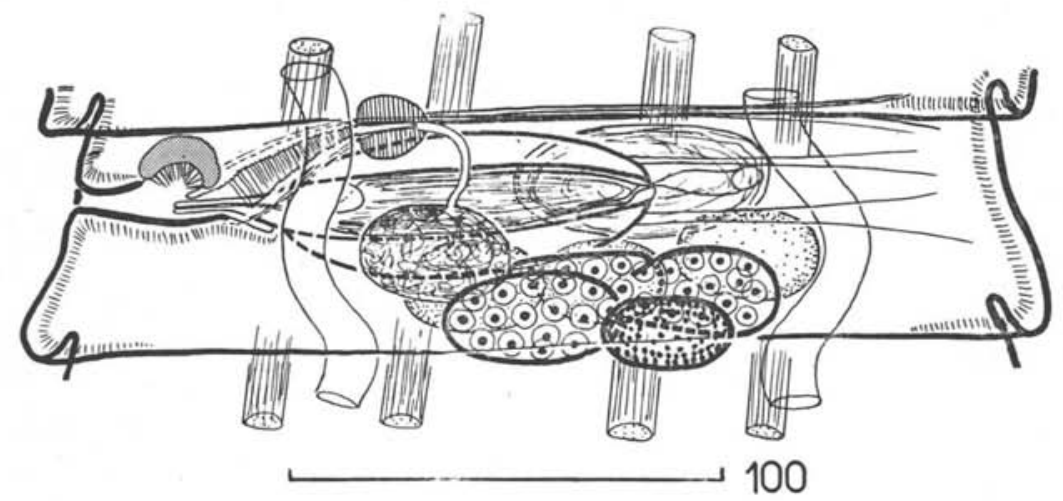

Fig. 45. - $H$. $(H$.$) capetownensis n. sp. Reconstitution d'un anneau mûr$ d'un exemplaire très contracté. Vue ventrale.

soies fines de 6,5-7,5 $\mu$. L'atrium, court sur le strobile décontracté, forme un tube étroit de $60 \mu$ environ sur les strobiles contractés. Musculature : formée de deux assises de faisceaux musculaires longitudinaux sur chaque face : l'une externe formée de 25 faisceaux de 4-5 fibres fines, l'autre interne formée de 4 faisceaux de 6 à 9 fibres volumineuses. CANAUX OSMO-RÉGUlateurs poraux 


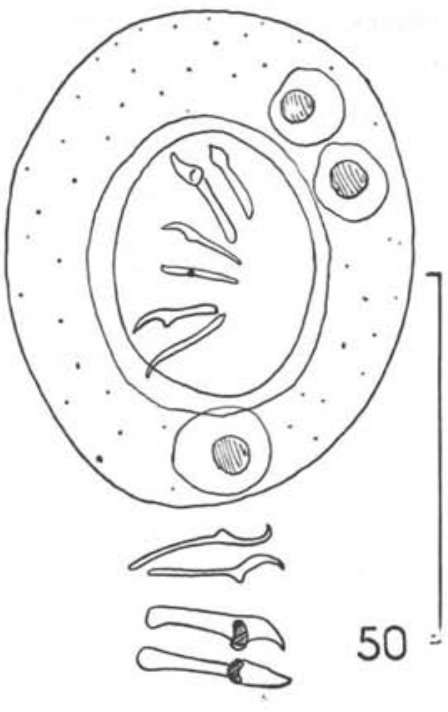

FIG. 46. $-H$. (H.) capetownensis n. sp. Eufs et crochets de l'embryon.

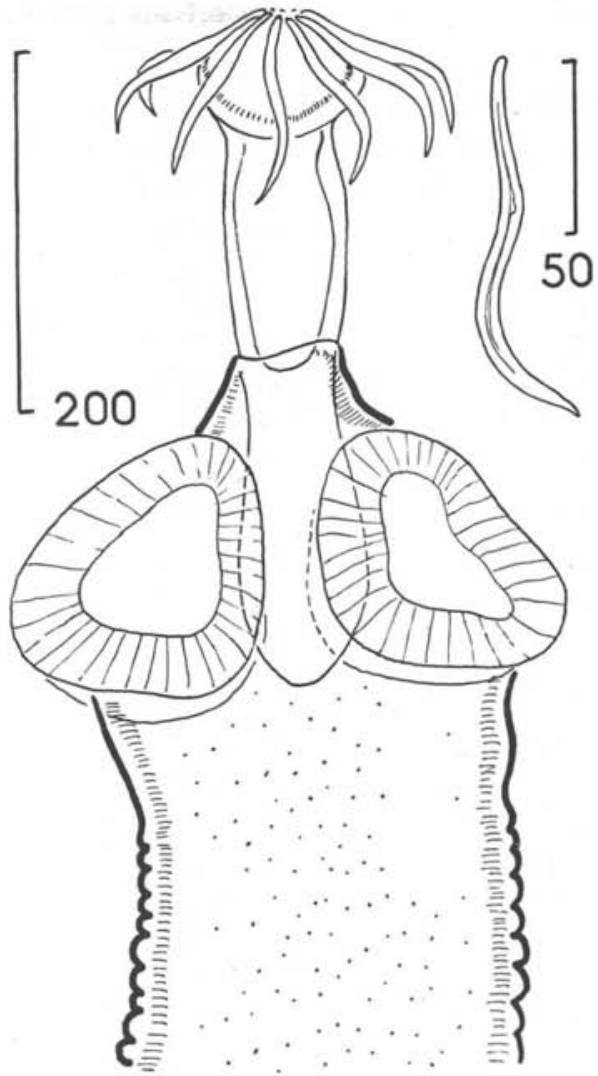

Fig. 47. $-H$. (H.) etaplesensis n. sp. Scolex à rostre évaginé et crochet du rostre.

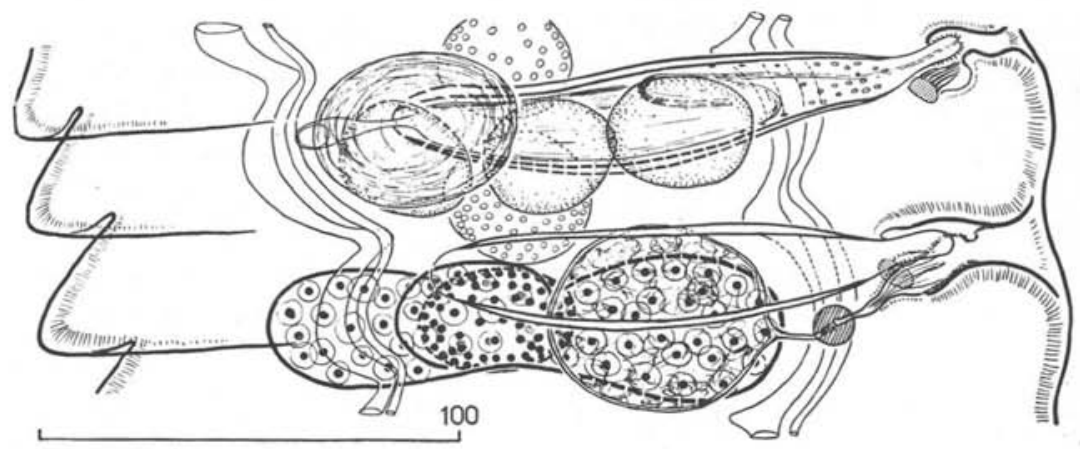

Fig. 48. - $H$. $(H$.$) etaplesensis n. sp. Anneaux mûrs. Vue dorsale. Anneau supérieur :$ système génital mâle et deux glandes vitellogènes. Anneau inférieur: système génital femelle et poche du cirre. 
en position ventrale par rapport aux conduits génitaux, les antiporaux étant en position dorsale par rapport à la poche du cirre.

Scolex (fig. 43). Forme générale : pyramidale à base rectangulaire par suite d'un aplatissement naturel dorso-ventral. Hauteur: $154 \mu$ (extrêmes : 145$185 \mu$ ), largeur maximale : $210 \mu$ (extr. : 186-225 $\mu$ ), épaisseur : $130 \mu$. Ventouses au nombre de quatre, ovalaires ou trianguliformes. Les deux ventouses d'une même face sont parfois en lorgnon. Diamètre : $100 \mu$ (extr. : 93-114 $\mu$ ) ; bord musculeux : 13-18 $\mu$. Absence d'armature. Rostre rétracté : $190 \mu$ (extr. : $160-210 \mu$ ) de long $\times 75 \mu$ de large ; évaginé : $300 \mu$ de long $\times 53 \mu$ de large ; table apicale : $60 \mu$ de diamètre. Sac du rostre : $190 \times 75 \mu$. Une couronne de 10 crochets à long manche sans garde et non plans (lame sinueuse) : 100 à 108-110 $\mu$. Epaisseur au niveau de la garde : $10 \mu$. Longueur du manche : $43,5 \mu$ (extr. : 40 à $45 \mu$ ).

Anneaux (fig. 44 et 45 ).

\section{A) Système génital mâle}

Testicules : au nombre de trois, situés à la partie postérieure et dorsale du proglottis, le testicule médian semble être le plus postérieur, tout au moins dans les anneaux jeunes, non contractés; sinon, ils se situent en ligne. Ils sont régulièrement sphériques ou ovalaires. Taille : $30 \times 25 \mu$, dans un anneau relâché de $140 \times 77 \mu$, à poche du cirre de $100 \times 30 \mu ; 44 \times 15 \mu$ dans les anneaux contractés, à leur maximum de développement (anneaux de $310 \mu \times$ $25 \mu$, à poche du cirre de $90 \times 36 \mu$ ).

POCHE DU CIRRE : à son maximum de développement vers les $130^{\circ}-150^{\circ}$ anneaux, elle mesure de 80 à $115 \mu$ de long $\times 35-40 \mu$ de diamètre maximal. De forme ovoïde allongée, elle est située parallèlement au bord antérieur de l'anneau, ne franchissant pas les canaux osmo-régulateurs antiporaux dans les anneaux rétractés ; ses parois sont formées de fibres musculaires longitudinales hélicoïdales très épaissies $(10 \mu)$. A sa partie postérieure, s'insèrent quelques fibres rétractrices transverses qui la rattachent au bord proglottidien antiporal. Dans les strobiles très contractés, les poches sont légèrement décalées les unes par rapport aux autres et alternantes: les poches des anneaux pairs se trouvant plus ventrales et plus porales, celles des anneaux impairs plus dorsales et plus antiporales, repoussant d'autant les glandes génitales.

VÉSICULE SÉminAlE INTERNE tubulaire, droite, décrivant ou non une courte boucle avant de former le canal éjaculateur. Absence de différenciation tubulochitineuse du canal éjaculateur.

CrRRE : court, en forme de simple cône effilé, sans renflement, apparemment totalement inerme. Taille : $25-30 \mu \times 6,5 \mu$ à l'état évaginé à l'intérieur de l'atrium génital.

VÉSICULE SÉminALE EXTERNE située dorsalement par rapport à la poche du 
cirre, à cheval sur l'axe longitudinal de l'anneau ; ovoïde, 50-65 $\times 25 \mu$ dans son état de développement maximal. Le canal déférent qui unit les deux vésicules mâles demeure peu visible sur toutes les préparations.

\section{B) Système génital femelle}

Différenciation : le vagin constitue le premier organe formé. Nettement plus tard, apparaissent ovaire et vitellogène, bien visibles dans quarante à cinquante anneaux. Dans la plupart des strobiles contractés, ces deux glandes sont rejetées du côté antiporal à l'extrémité de la poche du cirre.

OvarRe : postérieur, ventral, trilobé en lobes sensiblement égaux. Taille maximale : $80 \times 25 \mu$.

VITELlogèNE : postérieur à l'ovaire, ovoïde, non lobé. Taille maximale : $32 \times 20 \mu$.

RÉCEPTACLE SÉminAL: postéro-ventral à la poche du cirre, ovoïde ; $35 \times 25 \mu$.

VAGIN : antérieur et ventral par rapport au système mâle correspondant. Depuis le réceptacle séminal jusqu'à l'atrium génital, il mesure 90-100 $\mu$ de long environ. Du réceptacle séminal naît un tube étroit d'une quarantaine de microns; franchit ensuite un volumineux sphincter musculeux de $15 \mu$ sur $13 \mu$ de diamètre, où il se rétrécit avant de s'évaser en un tube conique de plus large section, à parois épaissies plissées transversalement, formant des papilles, long de 40 à $60 \mu$, venant s'aboucher dans l'atrium génital. Il n'y a aucune formation ampullaire comme chez $H$. nitida ou $H$. charadrii. A la partie postérieure du sphincter vaginal, s'insère une volumineuse fibre rétractrice transverse, dont l'autre extrémité s'attache au niveau de la commissure intersegmentaire antérieure antiporale; cette musculature est nettement plus apparente que son homologue de la poche du cirre.

\section{C) Anneaux gravides}

TAILLE à l'état contracté : $230 \times 53 \mu$ à $290 \times 80 \mu$ environ.

UTÉRus sacciforme, d'abord comprimé du côté antiporal, puis envahissant tout l'anneau, à l'exception des champs latéraux. Ne contient qu'un petit nombre d'œufs volumineux (une quinzaine). Les anneaux portant les œufs mûrs doivent se détacher du strobile au fur et à mesure de leur maturation, car ils sont réduits au nombre de 2 ou 3 .

Eufs régulièrement ovoïdes, mesurant $80 \times 64 \mu$ en moyenne ; embryon hexacanthe de $38 \times 30 \mu$ de diamètre ; crochets de $18-20 \mu$ de long, de morphologie hétérogène, la paire médiane différant des paires extrêmes (fig. 46). Coque externe chitinoïde mince $(1 \mu)$, non colorable par le carmin. 


\section{Discussion.}

Les espèces du sous-genre Hymenolepis à crochets falciformes de plus de $100 \mu$ sont au nombre de quatre : $H$. crocethia (Belop.), H. etaplesensis n. sp., $H$. moghensis Inamdar et $H$. nitida (Krabbe). Elles diffèrent toutes de l'espèce décrite : $1^{\circ}$ Les deux dernières sont dépourvues de sac accessoire. $2^{\circ}$ Le sacculus d'H. etaplesensis est plus rudimentaire, mais à soies plus longues ; son cirre est finement épineux, son vagin dépourvu de papilles; les crochets de son embryon hexacanthe sont plus courts $(11-12 \mu$ au lieu de 18-20 $\mu$ ). $3^{\circ} \mathrm{H}$. crocethia Belop., bien que très voisine, s'en différencie néanmoins : a) par la taille plus grande des anneaux et de leurs organes (le sac accessoire mesure $30 \times 13 \mu$ et les soies, $10-12 \mu$ ); b) par l'armature du cirre et sa forme ; $c$ ) par la position constante du sac accessoire en arrière de l'atrium (il est toujours antérieur dans l'espèce décrite), ces positions étant indépendantes de l'état de contraction des strobiles.

En conséquence, nous considérons cette espèce comme nouvelle et la nommons Hym. (Hym.) capetownensis, pour rappeler son origine géographique.

\section{3) HYMENOLEPIS (HYM.) ETAPLESENSIS n. sp.}

Région paléarctique: Europe (Baie de Canche, Pas-de-Calais, France).

Hôtes: Limosa lapponica (L.): une autopsie, septembre 1957; Crocethia alba (Pall.) : une autopsie, septembre 1959.

Matériel d'observation : trois scolex et des fragments de six à sept vers. Coupes histologiques.

\section{Description de l'espèce.}

Strobile de petite taille, $10-15 \times 0,3 \mathrm{~mm}$. Les anneaux ont une section presque circulaire, ce qui rend le Cestode épais.

Scolex : de forme pyramidale à base rectangulaire, par suite d'un aplatissement naturel dorso-ventral. Rappelle celui de $H$. charadrii. Petit : $160 \times 160$ $180 \mu$ de largeur maximale au niveau des ventouses. Ventouses inermes, profondes, à bord musculaire bien marqué, plutôt trianguliformes, de 96-106 $\times$ $90 \mu$. Disposées deux par deux sur chaque face du Cestode. Rostre invaginé : $160 \times 60 \mu$; le fond du sac atteint le niveau du bord postérieur des ventouses ; rostre évaginé : $310 \mu$, sur $30 \mu$ de diamètre à la sortie de l'étui du scolex ; table apicale de $80 \mu$ de diamètre où s'insèrent les crochets (fig. 47).

CROchets falciformes, disposés en une couronne de dix; 105-112 $\mu$, sur $8 \mu$ d'épaisseur au niveau de la garde.

Cou court $(150 \times 150-200 \mu)$, les premières segmentations apparaissant très vite.

NOMBRE D'ANNEAUX: approximativement une centaine d'anneaux très im- 
matures formant cou, 100 anneaux mâles, 75 anneaux mûrs hermaphrodites, 70 gravides, soit 350 anneaux au total, comptés sur une chaîne contractée de $10 \mathrm{~mm}$.

Musculature longitudinale interne formée sur chaque face de la chaîne de quatre volumineux faisceaux de $12-15 \mu$ de diamètre, non anastomosés. La couche musculaire externe est formée de 20 à 25 faisceaux plus petits $(3-4 \mu$ de diamètre).

Canaux osmo-RÉGulateurs ventraux par rapport aux conduits génitaux; 10 et $3 \mu$ de diamètre environ. Absence d'anastomose transverse.

ATRIUM GÉNITAL antérieur, unilatéral et dextre. Relativement profond sur les anneaux contractés. Inerme. Pourvu dorsalement d'un sacculus accessorius rudimentaire, de $8-11 \mu \times 5-6 \mu$, du type « peigne atrial », garni d'un pinceau d'une vingtaine de longues soies de $13 \mu$, déjà bien visibles dans les anneaux jeunes (fig. 49).

Anneaux (fig. 48).

$1^{\circ}$ AnNenux Jeunes : l'atrium génital y apparaît ampullaire et relativement volumineux $(10 \mu$ de diamètre), par rapport à la hauteur de l'anneau $(20 \mu)$.

$2^{\circ}$ AnNeaux mâles: $212 \times 30 \mu$. L'ébauche des testicules se dispose très diversement; le médian étant le plus dorsal et le testicule antiporal paraissant généralement plus réduit que les deux autres.

$3^{\circ}$ AnNeaux mûrs : taille : $313 \times 30 \mu$ ou $230 \times 40 \mu$.

- Système reproducteur mâle : trois testicules sphériques, postéro-dorsaux, toujours en ligne (type G), de $25 \mu$ de diamètre jusqu'à $40 \times 28 \mu$ à maturité complète, juste avant la ponte. Peu persistants, ils involuent pour disparaître vers le $10^{\circ}$ anneau gravide.

Poche du cirre antérieure et transverse, parallèle au bord antérieur du proglottis. Très musculeuse, fusiforme, formée de 20-21 fibres hélicoïdales longitudinales épaisses $(6,5 \mu)$. Le fond de la poche atteint les canaux osmo-régulateurs antiporaux. Taille : $80-100 \times 25-30 \mu$. Rapport PC/ AM (anneau non aplati) $=1 / 2,5$ à $1 / 3$. Fibres rétractrices peu marquées. Peu déformable ; parfois en croissant plus ou moins accentué. La vésicule séminale interne occupe la quasi-totalité de la poche $\left(8 / 10^{\circ}\right)$; le canal éjaculateur naît à la partie porale de cette dernière, pour aborder le cirre après avoir parcouru une courte boucle; il ne semble pas présenter de différenciation tubulo-chitineuse.

Le cirre est observé dans la plupart des cas partiellement évaginé ; seule, la partie basale est extroversée ; il présente alors un aspect de bouteille et mesure $20-25 \mu \times 6,5$ à $8 \mu$ de diamètre au niveau d'une dilatation ampullaire garnie d'épines petites de 1,5-2 $\mu$ de long. Plus évaginée, cette dernière se prolonge d'une portion tubulaire, de $35 \mu \times 2,5 \mu$ de diamètre, garnie d'épines punctiformes de $0,5 \mu$. 


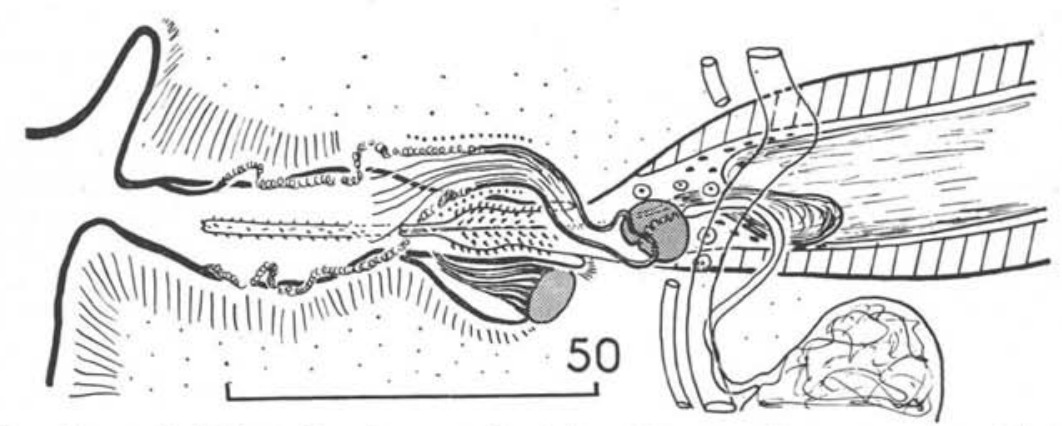

Fig. 49. - $H$. $(H$.$) etaplesensis n$. sp. Conduits génitaux terminaux et atrium génital. Vue ventrale.

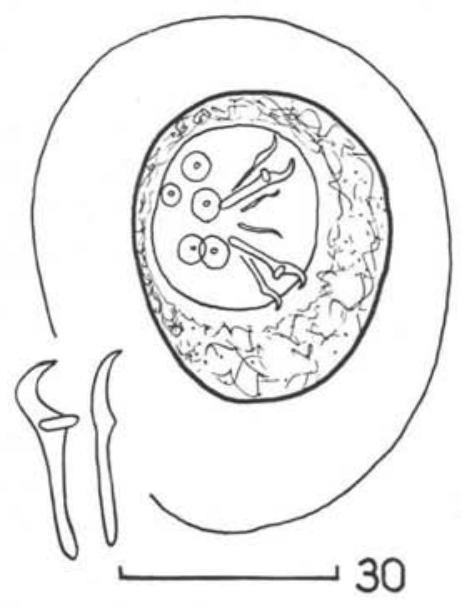

Fig. 50. - H. (H.) etaplesensis n. sp. Euf mûr et crochets de l'embryon (à plus fort grossissement).

Vésicule séminale externe ovoïde située dorsalement au-dessus de l'extrémité antiporale de la poche du cirre. Taille : $38-44 \times 25-30 \mu$. Le canal déférent intervésiculaire mesure $20-25 \mu \times 6-8 \mu$ de diamètre et n'est pas musculeux.

- Système reproducteur femelle : Ovaire postérieur, ventral et médian ; allongé transversalement, bi- ou trilobé, le lobe médian étant plus ou moins marqué ou confondu avec l'isthme. Taille : 90-100 $\times 30-35 \mu$. Vitellogène ovoïde ou sphérique, situé en arrière de l'isthme ovarien, dans l'axe longitudinal du strobile. Taille : 30-37 × 17-28 $\mu$. Le réceptacle séminal ne semble pas très fonctionnel : rarement visible dans les anneaux mûrs, il se situe néanmoins classiquement entre la poche du cirre en avant et le lobe poral de l'ovaire en arrière. Taille mesurée dans les anneaux gravides : $40 \times 20 \mu$ ou $45 \mu$ de diamètre suivant état de réplétion. Vagin situé ventralement en 

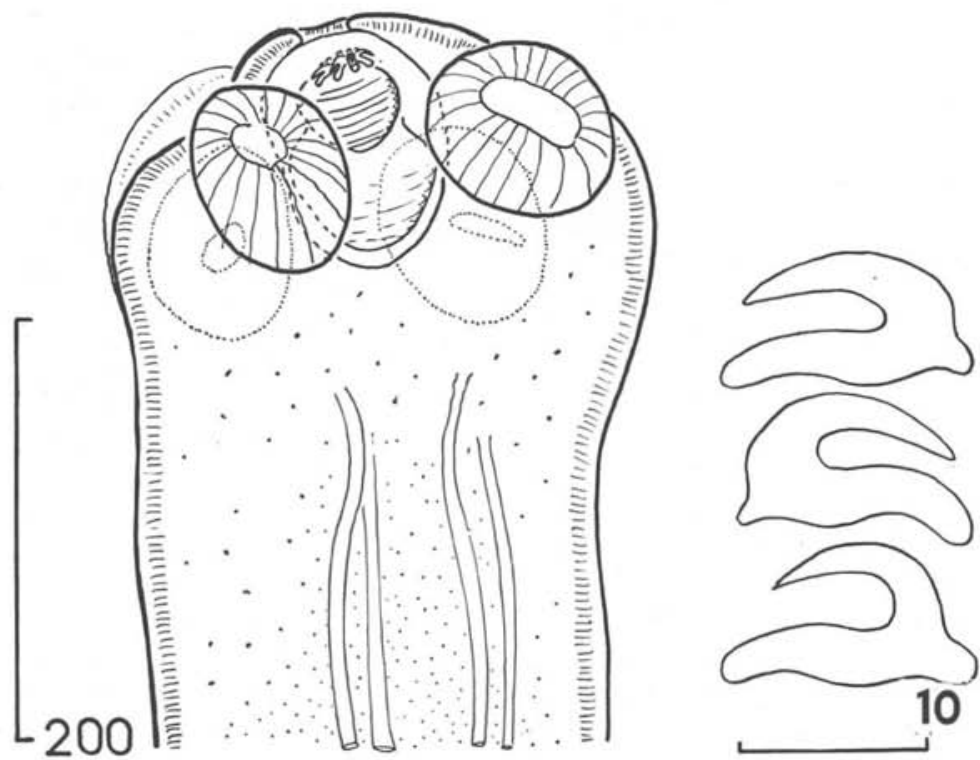

Fig. 51. $-H$. (H.) hughesi n. var. europaei. Scolex et crochets du rostre.

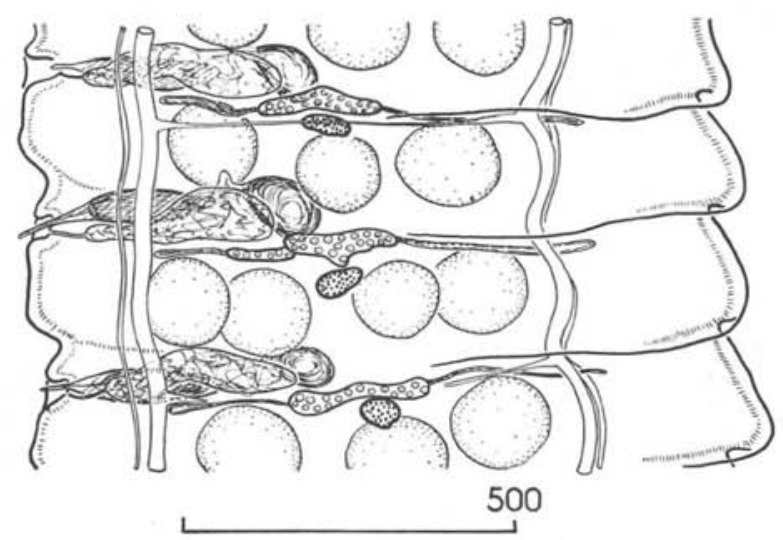

Fig. 52. - H. (H.) hughesi n. var. europaei. Anneaux mâles. Vue ventrale.

arrière de l'atrium génital et de structure hétérogène: portion distale copulatrice cylindrique, ou conique effilée, à parois chitinoïdes minces, longue de 20-30 $\times 7-13 \mu$ à l'embouchure. La pointe du cône s'engage dans l'axe d'un petit sphincter de 7-10 $\mu$ de diamètre, en y subissant une torsion sur elle-même. Portion proximale tubulaire non chitinisée, en rapport avec le réceptacle séminal. Il ne semble pas y avoir de fibres rétractrices insérées au niveau du sphincter atrial (fig. 49). 
$4^{\circ}$ AnNeaux gravides : taille : $315 \times 50-80 \mu$. Y disparaissent les testicules, le vitellogène et l'ovaire. Utérus sacciforme non lobé, à paroi nettement individualisable du parenchyme environnant comme un liseré plus colorable; envahit la totalité du parenchyme médullaire, franchissant ou non les C.O.R. ; dans les derniers anneaux de la chaîne, après disparition plus ou moins complète des œufs mûrs, selon un processus non élucidé, ses parois se collabent irrégulièrement. Il s'ensuit une diminution importante de la largeur de l'anneau.

Eufs mûrs : ovoïdes ou sphériques. Coque externe mince, de $50 \times 40 \mu$ à $70-80 \times 55-60 \mu$ suivant les strobiles. Coque interne nettement plus épaissie $(1,5 \mu)$, de $30 \times 23 \mu$ à $40 \times 30 \mu$, et non colorable. Embryon hexacanthe, de 23 à $28 \mu$ de diamètre. Crochets hétérogènes (les crochets internes des deux paires latérales étant plus développés que les quatre autres), de $11-12 \mu \times 2,5$ au niveau de la garde (fig. 50).

\section{Discussion.}

Parmi les quatre Cestodes cités dans la discussion de l'Hymenolepis précédent - les seuls à crochets de taille comparable à la présente espèce -, aucun ne possède un cirre, un sacculus et un vagin qui lui soient identiques. Nous considérons ce parasite comme nouveau et le nommons Hym. (Hym.) etaplesensis, pour rappeler le lieu de sa découverte.

\section{4) HYMENOLEPIS (HYM.) HUGHESI var. EUROPAEI nov. var.}

Région paléarctique: Europe (Baie de Canche, Pas-de-Calais, France).

Hôte : Charadrius hiaticula L.

Matériel d'observation: deux Cestodes (sept. 1959).

Toute la morphologie de ces deux Cestodes, dépourvus d'anneaux gravides mûrs, les identifie à l'espèce américaine ; seuls, le nombre des anneaux, la taille des strobiles sont plus élevés, alors que l'ovaire est un peu moins étendu ; les C.O.R. ventraux présentent des anastomoses transverses régulièrement disposées. Les caractéristiques principales de la souche européenne de $H$. $(H$.) hughesi Webster, 1947 sont les suivantes (fig. 51-54) :

STROBILE : jusqu'à $80 \times 0,95 \mathrm{~mm}(600$ anneaux, sans anneaux gravides mûrs). Scolex : $220 \mu$ de diamètre. Crochets chéliformes, $1 . X .13 \times 7,5 \mu$. Musculature longitudinale interne: 15-20 faisceaux par face. Atrium géNITAL peu développé, court, glabre, dépourvu de sac accessoire. POCHE DU CIRRE finement musculeuse, à fibres hélicoïdales nettes: 230-260 $\times$ 45-55 $\mu$. $\mathrm{PC} / \mathrm{AM}=1 / 4$ à $1 / 5$. Cirre petit, inerme $: 35 \times 10 \mu$. Testicules en ligne postérieurs à l'ovaire : $130-140 \times 100 \mu$. VÉsicule SÉMINALE externe médiane, petite : $115 \times 60 \mu$. OvaIRE médian, antérieur aux testicules, relativement peu 


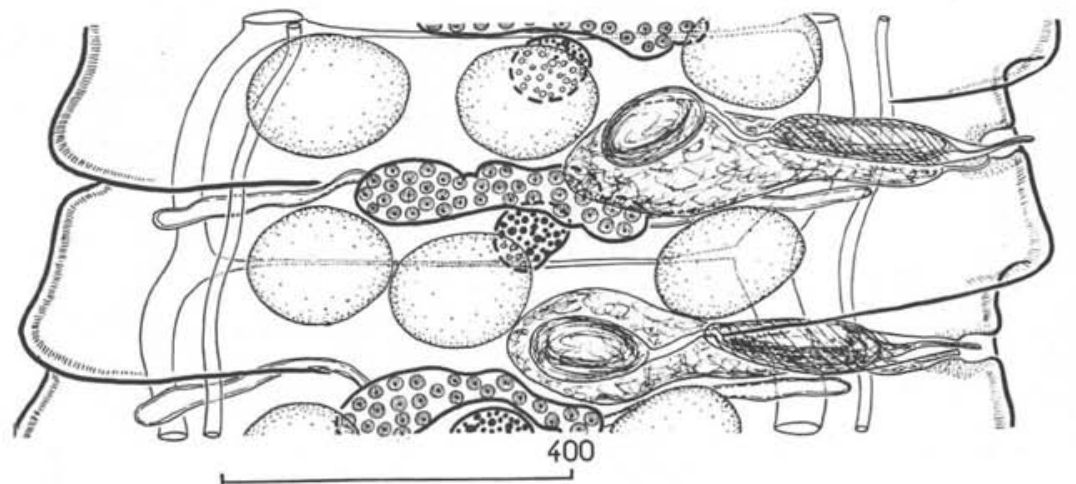

Fig. 53. - H. (H.) hughesi n. var. europaei. Anneaux mûrs. Vue dorsale.

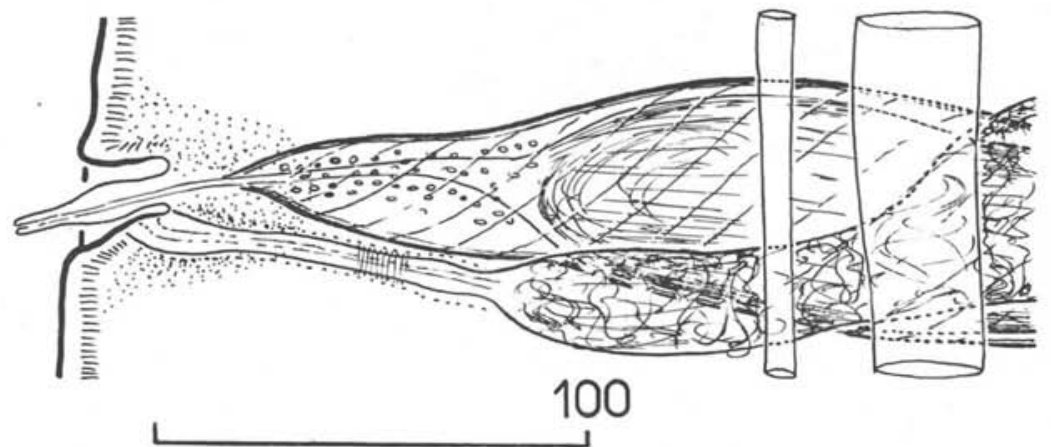

FIG. 54. $-H$. (H.) hughesi n. var. europaei. Conduits génitaux terminaux et atrium génital. Vue ventrale.
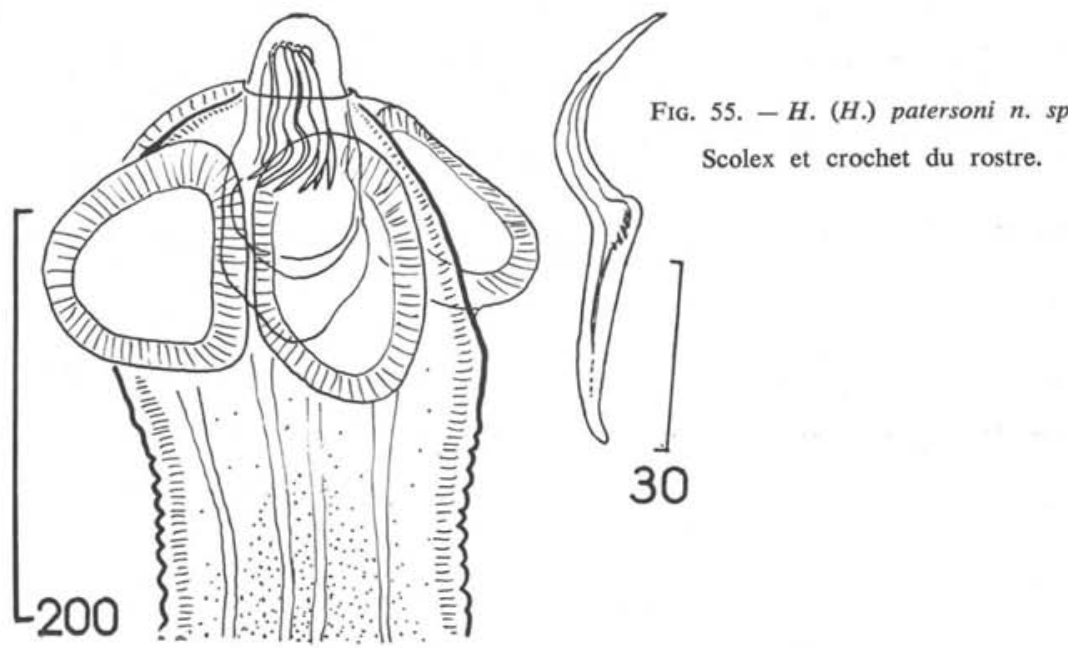
Scolex et crochet du rostre. 


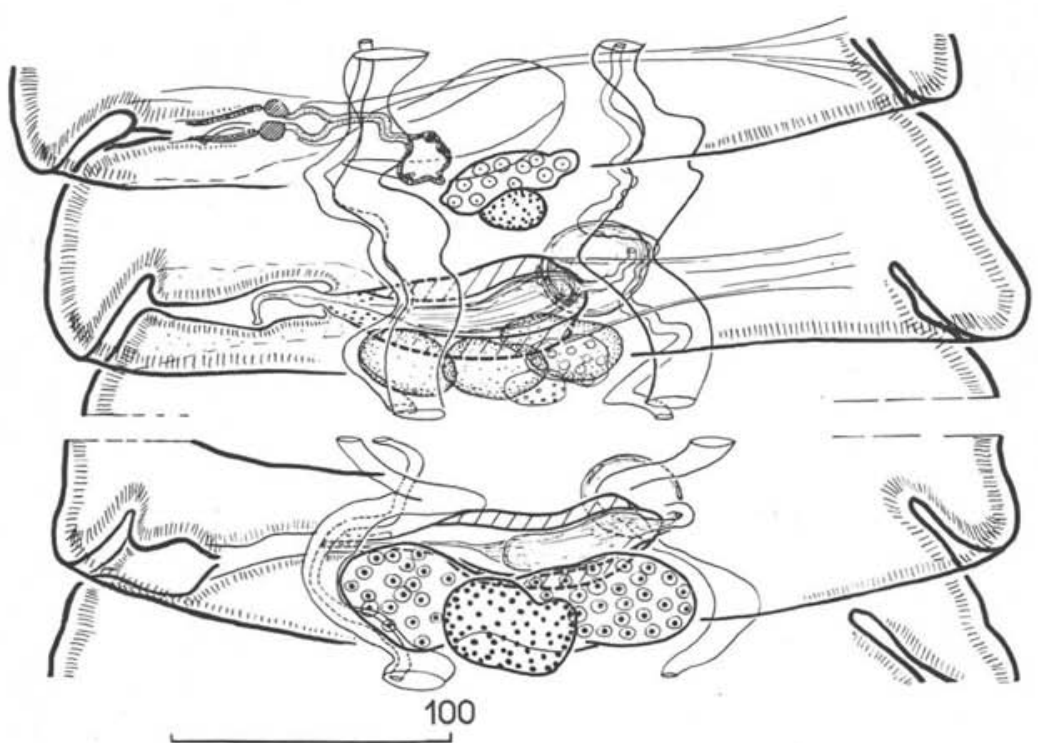

Fig. 56. - H. (H.) patersoni n. sp. Anneaux mâles. En haut, appareil génital femelle embryonnaire; en bas, appareil mâle.

Fig. 57. - H. (H.) patersoni $n$. sp. Anneaux mûrs, très contractés (la poche du cirre également très contractée, doit pouvoir normalement être plus longue). Vue ventrale.

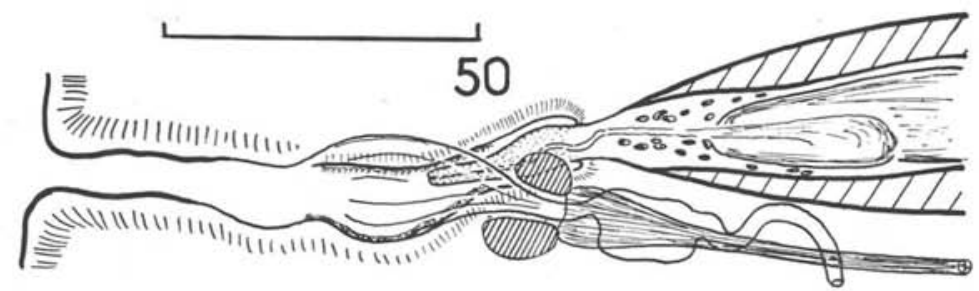

Fig. 58. $-H$. $(H$.) patersoni n. sp. Conduits génitaux terminaux et atrium génital. Vue ventrale. Cirre en pointillé.

FiG. 59. $-H$. $(H$.$) patersoni n . s p$. CEuf immature.

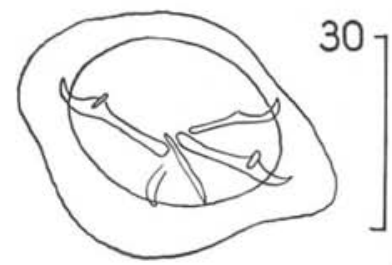




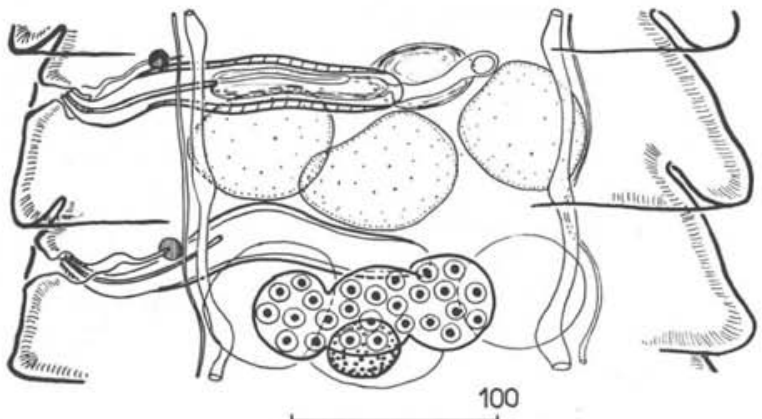

FIG. 60. - H. (H.) sp. inq. $\mathrm{n}^{\circ}$ 1. Anneau mûr. Système mâle et femelle. Vue ventrale.

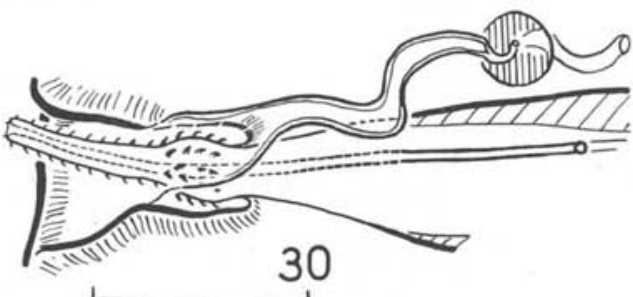

FIG. 61. - H. (H.) sp. inq. $\mathrm{n}^{\circ}$ 1. Atrium génital et conduits génitaux terminaux. Vue ventrale.

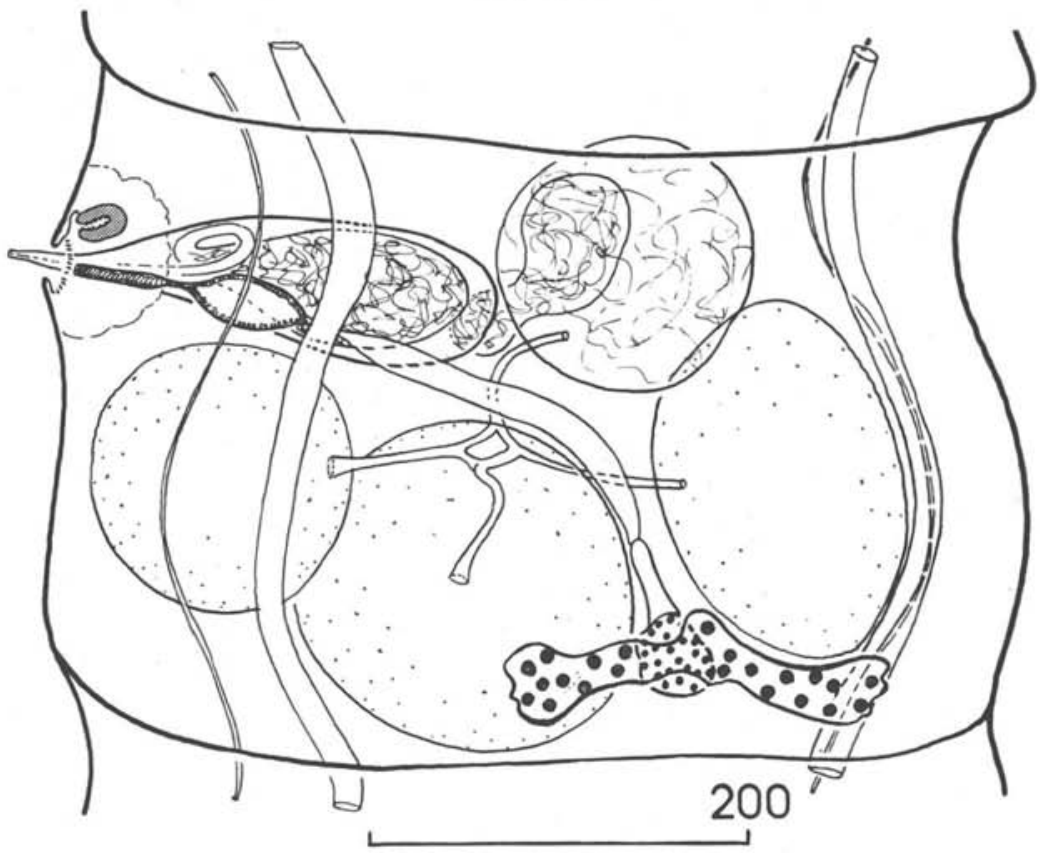

FIg. 62. - H. $(H) s$.$p . inq. \mathrm{n}^{\circ}$ 2. Anneau mâle. Vue ventrale. 


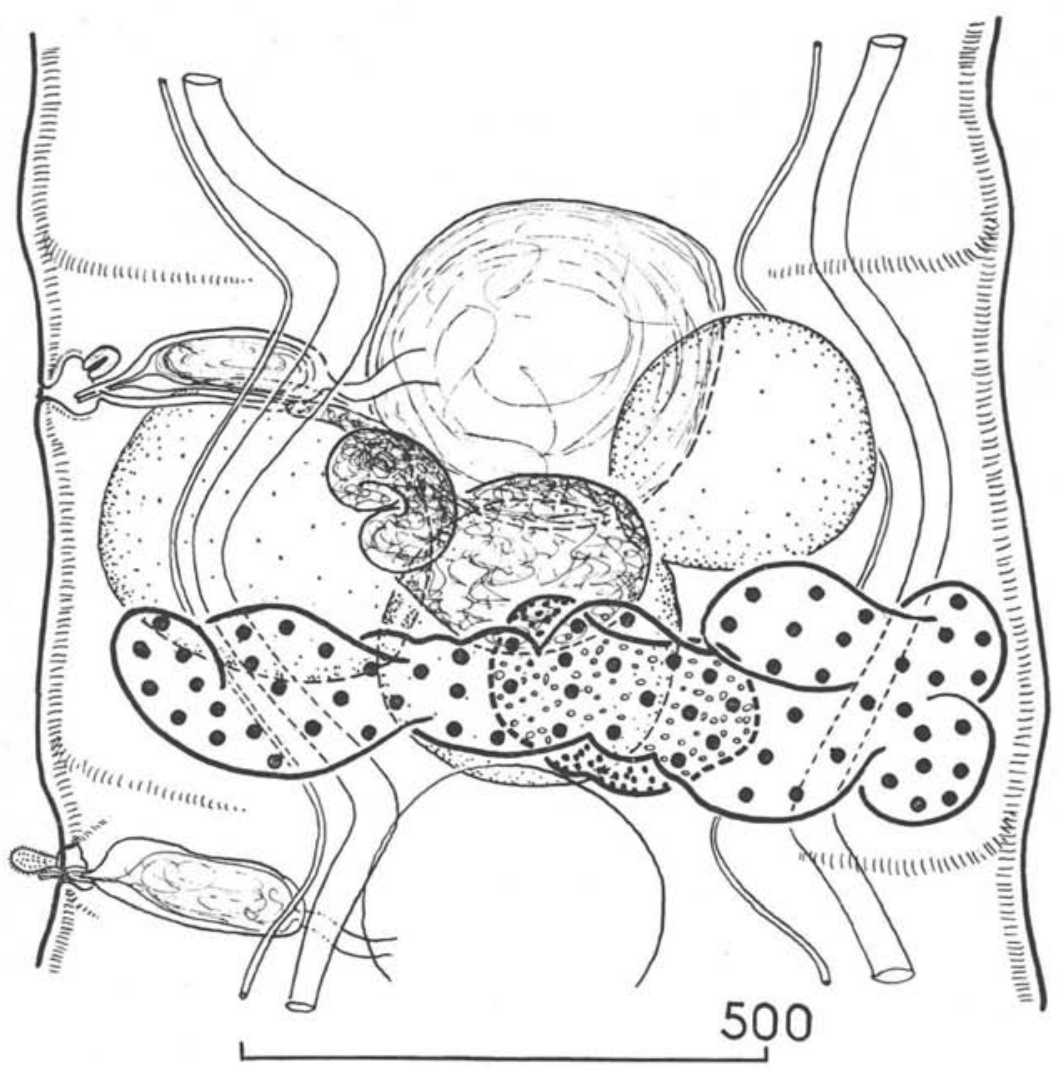

FIg. 63. $-H$. (H.) sp. inq. $\mathrm{n}^{\circ}$ 2. Anneau mûr. Vue ventrale.

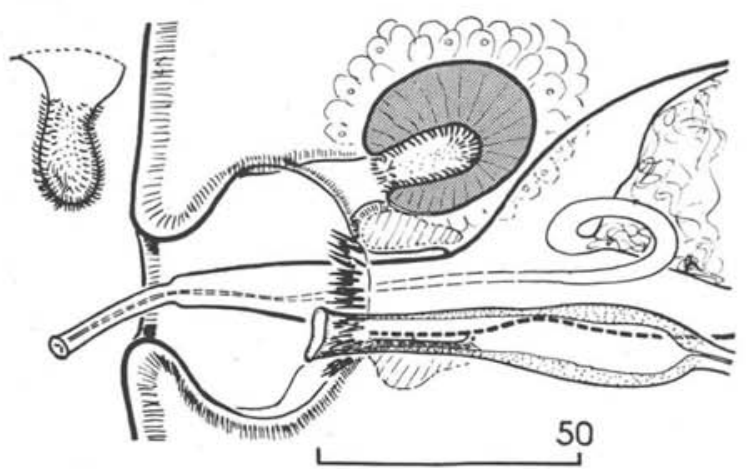

Fig. 64. $-H(H$.$) sp. inq. \mathrm{n}^{\circ}$ 2. Conduits génitaux terminaux et atrium génital. 
développé : $260 \times 50-60 \mu$. VITELlOGÈNE globuleux, petit, situé indifféremment à droite ou à gauche du testicule médian : $90 \times 50 \mu$. RÉCEPTACLE SÉmINAL volumineux, piriforme, dépassant du côté poral les C.O.R. : $360 \times 100$ $130 \mu$. VAGIN tubulaire grêle, dépourvu de dilatation, sphincter ou fibres rétractrices: $65-80 \times 8-10 \mu$. UTÉrus jeune, tubulaire, dépassant latéralement les C.O.R. ; utérus gravide sacculaire multilobé. Testicules persistants. Eufs inconnus.

\section{5) HYMENOLEPIS (HYM.) PATERSONI n. sp.}

nec H. nitida (Krabbe) sensu Baer, 1956

Hôte : Crocethia alba (Pall.).

Date de récolte : novembre 1955 (Mlle N.F. Paterson leg. Baer).

Localisation géographique: Capetown (Afrique du Sud).

Matériel de description: six strobiles complets, à l'exception des tout derniers anneaux très mûrs; colorés au carmin et montés au baume. Coupes histologiques.

\section{Strobile.}

TAILlE : longueur totale : 20 à $25 \mathrm{~mm}$, contracté, soit 3 à $4 \mathrm{~cm}$ en extension ; largeur maximale : 400 à $450 \mu$ au niveau des anneaux mûrs $\times 45$ à $50 \mu$ de hauteur; les anneaux gravides paraissent devoir être un peu moins larges $(300-350 \mu \times 65-80 \mu)$ et plus hauts. Nombre d'anneaux : 480 environ (y compris les anneaux jeunes du cou), se décomposant comme suit: 150 à 200 anneaux immatures sur une longueur de 1,5 à $2 \mathrm{~mm} ; 80$ anneaux mâles ; une centaine d'anneaux mûrs mâles et femelles; 160 anneaux gravides.

PORES GÉNITAUX: unilatéraux dextres.

Atrium GÉNITAL : dépourvu de sacculus accessorius et d'épines. Long de 40 à $70 \mu$ suivant la rétraction de la chaîne.

Musculature : comportant deux assises de faisceaux musculaires longitudinaux sur chacune des faces : l'une externe, formée d'une quarantaine de fins faisceaux longitudinaux de 1 à 2 fibres; l'autre, interne, formée de 4 faisceaux de grosse section, avec une dizaine de fibres chacun.

CANAUX OSMO-RÉGUlateurs : en position ventrale par rapport aux conduits génitaux sexuels; il existe une fine anastomose transverse à la partie postérieure de chaque anneau entre les canaux osmo-régulateurs ventraux.

Scolex (fig. 55).

Forme générale : pyramidale à base rectangulaire par suite d'un aplatissement naturel dorso-ventral. Hauteur : $150 \mu$ (extrêmes : 130-170 $\mu$ ). Largeur à la base : $190 \mu(160$ à $240 \mu)$. Epaisseur : $170 \mu$.

VENTOuSEs légèrement ovalaires ou trianguliformes. Les deux ventouses 
d'une même face sont parfois en lorgnon. Diamètre: $108 \mu \times 90 \mu$ de large (extrêmes: $95-130 \mu$ ). Bord musculeux : $13 \mu$ en moyenne. Inermes.

RosTRE rétracté : $140 \times 60 \mu$ (extr. : 130-160 $\mu \times 50-70 \mu$ ). Jamais observé évaginé sur les exemplaires.

CROCHETS : une couronne de 10 crochets falciformes à long manche, à garde réduite, et non plans (lame très sinueuse). Taille : $70 \mu$ (extrêmes : 68-71 $\mu$ ). Epaisseur au niveau de la garde : $9 \mu$. Longueur du manche, garde comprise : 38 à $39 \mu$.

Cou : la segmentation commence immédiatement en arrière du scolex.

\section{Système génital mâle (fig. 56).}

Testicules : au nombre de trois, situés à la partie postérieure et dorsale du proglottis ; le testicule médian semble être le plus postérieur, tout au moins dans les anneaux non contractés. Ils sont régulièrement ovalaires et mesurent $30 \times 16 \mu$ en moyenne à leur état de plus complet développement.

POCHE DU CIRRE : dans les anneaux mûrs (vers le $300^{\circ}$ anneau), la poche du cirre mesure en moyenne $70 \mu \times 30 \mu$ de diamètre (dans des anneaux de $350 \mu$ de large). Dans les anneaux âgés, elle peut atteindre jusqu'à $90 \times 38 \mu$, avec une paroi épaisse de $9 \mu$. Piriforme, allongée, elle est située parallèlement au bord antérieur de l'anneau, son fond dépassant légèrement l'axe longitudinal du Cestode à l'état semi-contracté ; décontracté, il est possible qu'elle se rapproche davantage des canaux osmo-régulateurs antiporaux. Sa paroi est formée de fibres musculaires longitudinales hélicoïdales épaissies $(7-9 \mu)$. Les cellules prostatiques sont condensées dans la partie porale de la poche; son fond est relié par quelques fibres rétractrices à la paroi antiporale de l'anneau.

VÉSICULE SÉMINALE INTERNE tubulaire, apparemment droite; il ne semble pas exister de différenciation tubulo-chitinoïde du canal éjaculateur.

CIRRE court, glabre, très légèrement renflé à la base. Taille : $25-35 \times 6-8 \mu$ à l'intérieur de l'atrium génital.

VÉSICULE SÉMINALE EXTERNE : située dorsalement par rapport à la poche du cirre et à son extrémité antiporale ; sphérique ou légèrement ovoïde, $25 \times 30 \mu$. Le canal déférent qui réunit les deux vésicules est court $(20 \mu)$.

Système génital femelle (fig. 57).

Situé dans l'axe longitudinal du strobile, le système génital femelle prend naissance ventralement un peu en arrière des deux testicules poraux.

Ovaire : postérieur, ventral, médian; sa structure est difficile à préciser, vu la contracture des anneaux (bilobé ?). Taille maximale: $76 \mu$ à $110 \mu \times$ $25 \mu-40 \mu$.

Vitellogène : postérieur à l'ovaire, ovalaire. Taille maximale : 40-50 $\mu \times$ 20-35 $\mu$. 
RÉCEPTACLE SÉMINAL : ovoïde et situé à mi-distance entre l'ovaire et l'atrium génital, en arrière de la poche du cirre. Dans les anneaux jeunes, il mesure 20-25 $\times 13-18 \mu$, pour atteindre $40 \times 65 \mu$, son complet développement dans les anneaux mûrs.

VAGIN : ventral par rapport au système mâle correspondant. Depuis le réceptacle séminal jusqu'à l'atrium génital, il mesure 70 à $80 \mu$ de long environ. Du réceptacle séminal, naît un tube étroit de $20-25 \mu$ ) (4-5 $\mu$ de diamètre ; il s'enfle ensuite en une chambre ovoïde à parois chitinoides, de $13 \times 7 \mu$, nettement figurée sur certaines chaînes, sans présenter de tortillons analogues à ceux d'H. charadrii Yamag., 1935 (cf. Deblock et coll., 1960). Presque immédiatement au contact de cette dilatation, le canal se rétrécit sur 8-10 $\mu$, pour franchir un sphincter musculeux volumineux très bien figuré, sensiblement sphérique, de $13-15 \mu$ de diamètre sur 6-7 $\mu$ d'épaisseur. Il se termine à l'atrium génital en s'évasant en un tube conique de $25 \mu \times 15 \mu$, à la paroi renforcée de striations chitinoïdes longitudinales; l'état des exemplaires étudiés ne nous a pas permis de confirmer la présence de papilles vaginales (fig. 58). A la partie postérieure du sphincter vaginal, s'insère une volumineuse $(2,5 \mu$ de diamètre) fibre rétractrice transverse, qui va s'insérer au niveau de la commissure intersegmentaire antérieure antiporale.

\section{Anneaux gravides.}

Nombre : 80-90. Taille : $310 \times 80$ à $100 \mu$. Utérus sacciforme envahissant la totalité de l'anneau, à l'exception des champs latéraux. Ne contient qu'un petit nombre d'œufs (environ une quinzaine).

G.UFs. L'œuf réellement mûr est inconnu (la coque externe n'est nettement formée dans aucun des strobiles). Embryon hexacanthe ovoïde, de 25-30 $\mu \times$ 17-19 $\mu$, pourvu de six crochets de structure hétérogène, de $18 \mu$ de long sur $3,8 \mu$ de hauteur (fig. 59).

\section{Discussion.}

Dépourvu de sac accessoire et garni de crochets de $70 \mu$ de long, cet Hymenolepis (Hym.) ne peut être comparé qu'à Hym. charadrii Yamag. Il n'en a ni le cirre, ni le vagin; la même remarque est valable pour $H$. lauriei et pour H. nitida qui, par ailleurs, possèdent des crochets plus longs.

Il faudrait pouvoir réexaminer la structure des conduits génitaux des types de H. magnisaccis Meggitt, 1927 pour discuter utilement de leur identité avec l'espèce décrite ; c'est pourquoi nous la considérons, au moins provisoirement, comme nouvelle et la dédions à Mlle Paterson qui l'a récoltée. 


\section{D) DESCRIPTION DE SPECIES INQUIRENDAE DE LA FAUNE DE FRANCE}

\section{1) HYMENOLEPIS (HYM.) spec. inq. $\mathrm{n}^{\circ} 1$}

Hôte: Crocethia alba (Pallas), Baie de Canche, septembre 1956.

Un fragment de 223 anneaux dépourvu de scolex et d'anneau gravide montre l'anatomie représentée aux fig. 60 et 61 . Strobile de 2,5 à $3 \mathrm{~cm}$ environ. ANNEAU MûR: $360 \times 80 \mu$. POCHE DU CIRRE musculeuse, de $130-160 \mu \times$ 25-30 $\mu$. Différenciation chitineuse du canal éjaculateur. CIRRE de 30-40 $\mu \times$ $8-10 \mu$, finement épineux (épines de 1,5 à $2 \mu$ ), mesurant vraisemblablement $70 \mu$ à l'état d'évagination totale. Testicules en position du type $\mathrm{D}$, de $53 \mu$ de diamètre ou de $75-80 \times 55 \mu$. VÉsicule sÉminAle externe de $53 \times 26 \mu$. OVAIRE de 100-110 $\mu \times 47-50 \mu$, trilobé; VITEllogène de 35-40 $\times 25 \mu$. VAGIN de $45 \times 6 \mu$, conique, dépourvu de papilles et de dilatation, occlus d'un sphincter musculeux de $10 \mu$ de diamètre. Atrium génItal court, dépourvu d'épines et de sac accessoire. CANAux osmo-RÉGul.ATEURS dépourvus d'anastomose transverse. Musculature interne formée de plus de quatre bandes longitudinales. Ce Cestode semble ne se rapporter à aucune espèce décrite.

\section{2) HYMENOLEPIS spec. inq. $\mathrm{n}^{\circ} 2$}

[= Hymenolepis (Echinocotyle) hypoleuci Singh, 1952 ?].

Localisation géographique: Hondschoote, 1961 (Nord); Etang du Canet (Pyrénées-Orientales) (A.-G. Chabaud leg., 1956).

Hôtes: Tringa erythropus (Pallas); Tringa ochropus L.

Matériel de description: quatre strobiles, tous sans scolex, ni anneaux gravides mûrs.

Longueur totale des STRoBILES décontractés : 30 et $40 \mathrm{~mm} \times 0,82 \mathrm{~mm}$ de large; les exemplaires entiers gravides doivent atteindre vraisemblablement $60 \mathrm{~mm}$. On compte une centaine d'anneaux immatures, 50 anneaux mâles, 30 anneaux femelles et 17 anneaux gravides jeunes, soit 200 anneaux environ; la maturation des œufs apparaissant comme ralentie, le nombre total d'anneaux devrait s'élever à 250 au moins.

SCOLEX : inconnu.

Musculature longitudinale: formée de 40 à 50 fines fibres externes et de 15 à 20 faisceaux de fibres internes sur chacune des faces du Cestode.

Canaux eXCrÉteurs : une paire de canaux longitudinaux latéraux, ventraux par rapport aux conduits sexuels, mais dorsaux par rapport aux ailes de 
l'ovaire; dépourvus d'anastomoses transverses unitives, en dépit de quelques fibres musculaires qui pourraient le faire supposer. Le diamètre du ventral est de $40-45 \mu$; le dorsal est de $4 \mu$.

ATrIUM GÉNITAL : situé à la limite des premier et deuxième quarts antérieurs de l'anneau. Unilatéral dextre. Profond de 30 à $40 \mu$ dans les anneaux mûrs. Armé. L'armature décrit un cercle complet de 25-30 $\mu$ de diamètre ; entourant seulement l'orifice mâle, elle est formée de nombreux petits crochets, longs de 5-6 $\mu$ et disposés en deux rangées au moins. Le sacculus accessorius est disposé dorsalement, du côté antérieur par rapport à la poche du cirre. Invaginé, il mesure $38 \times 22-23 \mu$ de large ; ses parois épaisses de 6-7 $\mu$ ménagent dans l'axe une lumière de $25 \times 6 \mu$, armée de fines épines recourbées de $2,5 \mu$ de long. Evaginé, il a une forme massuée, longue de $25 \times 9 \mu$ de diamètre, le rétrécissement basal mesurant $6,5 \mu$ de diamètre et la base plus élargie, $11,5 \mu$ (fig. 64).

\section{Appareil reproducteur.}

a) ANNEAUX MÂles :

A peine plus larges $(470 \mu)$ que longs $(417 \mu)$. Rapport largeur/longueur $=$ $1 / 1,1$; ils sont donc presque carrés. Cette disposition s'atténue légèrement lors de la maturation du strobile (rapport $=1 / 1,6$ dans anneaux femelles et gravides immatures).

Protérandrie accusée. Testicules sphériques postéro-dorsaux de 210-260 $\times$ $180 \mu$, toujours disposés en triangle selon le type D (deux testicules latéraux antérieurs, le postérieur médian symétrique des deux autres). Les massifs embryonnaires des glandes femelles naissent ventralement à la limite postérieure des deux testicules antiporaux (fig. 62).

Les spermiductes bien visibles sont différemment anastomosés en avant du testicule médian.

Poche du cirre petite, plutôt piriforme, parallèle au bord antérieur de l'anneau, limitée par une paroi musculaire fine $(1,5 \mu)$, dont les fibres ne paraissent pas individualisées. Taille : $170 \times 70 \mu$. Elle dépasse légèrement les C.O.R. poraux, n'atteignant jamais l'axe longitudinal du strobile, même dans les anneaux jeunes.

Rapport : poche du cirre/anneau mâle $=1 / 2,7$, et dans les anneaux mûrs $=$ $1 / 3,3$, bien que la poche du cirre s'y allonge jusqu'à $250 \mu$.

Absence totale de fibres rétractrices.

Vésicule séminale interne située dans les $2 / 3$ postérieurs de la poche, le canal éjaculateur décrivant toujours une large boucle entre son départ du sommet poral de la vésicule séminale et son engagement dans l'axe du cirre.

Cirre entièrement glabre. De forme cylindro-conique, à demi-évaginé, long de $40 \mu \times 11,5 \mu$ de diamètre à la base. Complètement évaginé, atteint 70-80 $\mu$ par adjonction d'une portion plus effilée de $4 \mu$ de diamètre. 
Vésicule séminale externe ovoïde ou sphérique toujours située dans le prolongement de la poche du cirre, au niveau de l'axe du strobile; $220 \times 180$ ou $210 \mu$ de diamètre, à réplétion complète. Canal déférent externe souvent volumineux, très dilaté, non musculeux.

b) Anneaux femelles :

Taille : anneau contracté : $500 \times 200 \mu$; relâché : $800 \times 500 \mu$.

Ovaire bien développé, multilobé, ventral, $780 \times 180 \mu$, très allongé parallèlement à tout le bord postérieur de l'anneau et dépassant ventralement les C.O.R. de part et d'autre, ce qui semble assez exceptionnel chez les Hymenolepis de Charadrii. Le lobe antiporal est légèrement plus développé que l'autre à maturité, alors que ces lobes sont presque égaux à l'état jeune (fig. 63).

Vitellogène: situé dorsalement par rapport à l'ovaire. D'aspect variable : ovoïde, sphérique, bilobé, parfois polylobé, $140 \times 225 \mu$ ou $150 \mu$ de diamètre.

Réceptacle séminal de $280 \times 115 \mu$ à réplétion, situé dans l'axe du strobile entre l'ovaire et la vésicule séminale externe. En forme de poche piriforme, dont le sommet aminci est susceptible de parcourir une ou deux boucles dilatées en direction du vagin distal. De ce fait, la limite entre le réceptacle séminal et le vagin proximal n'est pas définie.

Vagin peu différencié, ne dépasse pas les canaux osmo-régulateurs ; long de 50-65 $\mu$, large de 7,5-10 $\mu$; dans sa partie initiale, se dilate légèrement jusqu'à $15 \mu$ avant de subir une diminution de calibre pour s'engager dans l'axe de fibres musculaires annulaires faiblement développées. Sa paroi n'apparaît pas chitinisée. Fibres rétractrices absentes.

Utérus gravide jeune multilobé, contenant de très nombreux œufs ; envahit tout le parenchyme de l'anneau, en dépassant latéralement les canaux osmorégulateurs du côté ventral.

\section{Diseussion.}

L'absence de scolex ne facilite pas la diagnose exacte de ce Cestode, vraisemblablement du sous-genre Echinocotyle; l'espèce anatomiquement la plus proche est $H$. (E.) hypoleuci Singh, 1952, à laquelle nous sommes tentés de la rapporter, en dépit de quelques différences, comme par exemple la taille des cirres $(70-80 \times 11 \mu)$ pour l'espèce française et $330 \mu$ pour l'espèce indienne. Par contre, la musculature interne de 15-20 faisceaux ne peut la faire rapporter à $H$. (E.) uralensis Clerc, 1902-1903, qui n'en possède qu'une de quatre.

\section{3) HYMENOLEPIS (ECHINOCOTYLE) spec. inq. $\mathrm{n}^{\circ} 3$}

[ = H. (Echino.) glareolae Singh, 1952 ?].

Un Cestode très immature de Charadrius hiaticula L. (Baie de Canche) montre l'ébauche de trois testicules disposés suivant le type D. Son scolex pré- 
sente les caractéristiques de l'espèce de Singh: $170 \times 140 \mu$ de large ; crochets falciformes de 64 à $73 \mu$; rostre invaginé de $170 \times 60 \mu$, dépassant légèrement le bord postérieur de ventouses du type Echinocotyle, de $100 \times 60 \mu$, armées sur leur bord de 15 à 18 éventails de 3 à 5 crochets de $4-5 \mu$ et de 4-5 rangs longitudinaux d'épines isolées, sur leur fond.

E) ESSAI DE CLÉ DIAGNOSTIQUE pour servir à la diagnose

\section{des espèces d'HYMENOLEPIS de CHARADRII}

\section{Abréviations :}

BMI $=$ bandes musculaires longitudinales internes $;$ COR $=$ canaux osmo-régulateurs $; \mathrm{Cr}$. = crochets du rostre $; \mathrm{OV} .=$ ovaire $; \mathrm{PC} / \mathrm{AM}=$ rapport de la longueur de la poche du cirre non contractée à la largeur de l'anneau mûr ; P. du C. = poche du cirre $;$ R.S. = réceptacle séminal $;$ S.A. $=$ sac accessoire $;$ T. ou Test. $=$ testicules.

- Crochets chéliformes $\ldots \ldots \ldots \ldots \ldots \ldots \ldots \ldots \ldots \ldots \ldots \ldots \ldots \ldots \ldots \ldots \ldots \ldots$

Crochets falciformes $\ldots \ldots \ldots \ldots \ldots \ldots \ldots \ldots \ldots \ldots \ldots \ldots \ldots$, B

Crochets claviformes $\ldots \ldots \ldots \ldots \ldots \ldots \ldots \ldots \ldots \ldots \ldots, \mathrm{C}$

- Rostre dépourvu de crochets. Scolex de grande taille $(1.250 \mu$ de diamètre). Testicules du type B. Parasite du rectum .. H. glandularis Fuhrm.

\section{A. Crochets chéliformes}

Une couronne de $68-70$ crochets de 9 à $12 \mu$. P. du C. $147 \mu$. PC/AM $=1 / 4 \ldots \ldots \ldots \ldots \ldots \ldots \ldots \ldots \ldots \ldots$ H. (H.) skriabinissima Krotov.

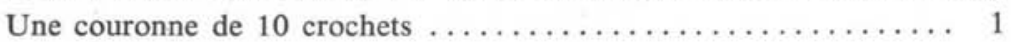

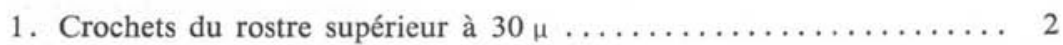
Crochets du rostre compris entre 18 et $23 \mu$. Disposition ovaire-testicules

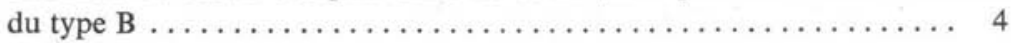

Crochets du rostre compris entre 8 et $15 \mu$. Testicules en ligne ... 6

2. [Crochets du rostre de $42-45 \mu$. Reste de l'anatomie inconnu ......... H. uliginosa (Krabbe) sp. inq.] Testicules en ligne ; Cr. de 38-46 $\mu$; ovaire légèrement devant les deux testicules poraux (type $\mathrm{H} 1$ ) $\ldots \ldots \ldots \ldots$ H. (H.) clandestina (Krabbe). Testicules antiporaux superposés (type B) $\ldots \ldots \ldots \ldots \ldots \ldots \ldots \ldots \ldots \ldots \ldots$

3. P. du C. longue $(>300 \mu)$, dépassant les COR poraux. $\mathrm{PC} / \mathrm{AM}=1 / 3$. Cirre puissant de $180 \mu$ fortement armé ; Cr. de $33 \mu \ldots \ldots \ldots \ldots \ldots$ H. (H.) hamasigi Yamag. 
P. du C. courte $(180 \mu)$, atteignant juste les COR. PC/AM = 1/6 à $1 / 10$. Cirre non remarqué. Cr. de $27-30 \mu \ldots$ H. (H.) annandalei Southwell.

4. Garde massive. Cr. de $21-23 \mu$. P. du C. courte $(180 \mu)$. PC/AM $=1 / 4-$ $1 / 5 \ldots \ldots \ldots \ldots \ldots \ldots \ldots \ldots \ldots \ldots \ldots \ldots \ldots \ldots \ldots \ldots \ldots \ldots \ldots \ldots$ (H.) chionis Fuhrm.

Garde mince. $\mathrm{P}$. du C. longue. $\mathrm{PC} / \mathrm{AM}=1 / 2$ à $1 / 3,5$; cirres épi-

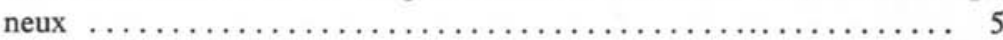

5. Cirre puissant $(210 \times 30 \mu)$, fortement épineux. Cr. de $20-23 \mu \ldots \ldots$. H. (H.) amphitricha (Rud.).

Cirre grêle, très finement épineux. $\mathrm{Cr}$. de $18-21 \mu$

H. (H.) capellae Baer.

6. a) Cr. de $13 \mu$. Vagins tubulaires courts. R.S. piriforme dépassant légèrement les COR poraux. Test. en ligne. Ovaire en avant des test. . 7 b) Cr. de 8-9 $\mu$ à garde spatulée. Reste de l'anatomie mal connu .... H. (H.) himantopodis (Krabbe) sensu Baer, 1940. c) Groupe mal connu de plusieurs espèces à test. en ligne dont les Cr. varient de 10 à $16 \mu \ldots \ldots \ldots \ldots \ldots \ldots \ldots \ldots .9$ (voir aussi $7 b$ ).

7. a) Cirres glabres, courts $(35 \mu)$, cylindro-coniques effilés $\ldots \ldots \ldots \ldots 8$

b) Cirre glabre, globuleux et pédiculé $(35-45 \times 25 \mu)$. P. du C. de 150 $200 \mu . \mathrm{PC} / \mathrm{AM}=1 / 6$ à $1 / 8$

........ Forme à 3 testicules d'Oligorchis paucitesticulatus Fuhrm. (crochets variant de 12 à $15 \mu$ ).

8. P. du C. de $145 \times 35 \mu ; \mathrm{PC} / \mathrm{AM}=1 / 5$; ovaire de $310 \mu$ H. (H.) hughesi Webster. P. du C. de $245 \times 50 \mu ; \mathrm{PC} / \mathrm{AM}=1 / 4$ à $1 / 5 ;$ ovaire de $260 \mu \ldots$. H. (H.) hughesi nov. var. europaei.

9. Species inquirendae (peut-être synonymes) :

a) crochets de $13-17 \mu$. Cirre de $100 \times 8 \mu$

H. recurvirostrae (Krabbe).

b) crochets de $10-15 \mu ;$ P. du C. courte $(100 \mu) ; \mathrm{PC} / \mathrm{AM}=1 / 8 \ldots$. H. recurvirostroides Meggitt.

c) crochets de $13-15 \mu$; P. du C. $120-180 \mu$; PC/AM $=1 / 10$. Eufs à membrane interne épaissie aux pôles

H. recurvirostrae sensu Joyeux et B., 1940.

d) crochets de $10-11 \mu ;$ P. du C. longue $(350-400 \mu) ; \mathrm{PC} / \mathrm{AM}=1 / 2$. Cirre finement épineux. Musculature interne de 8 à 10 faisceaux ...... H. tsengi Joyeux et Baer.

e) crochets de $8-9 \mu$. P. du C. très longue $(220-420 \mu) . \mathrm{PC} / \mathrm{AM}=1 / 1,5$. Cirre inerme. Vagin cilié à parois réfringentes aussi long que la P. du C. H. (H.) edouardensis (Baer, 1959) nom. nov. 


\section{B. Crochets falciformes}

Ventouses typiques d'Echinocotyle. Sac accessoire toujours présent ; atrium garni d'une couronne de petites épines. Strobiles de 2-5 mm . . 1 Ventouses typiques d'Hymenolepis. Atrium génital non spinulé ; présence

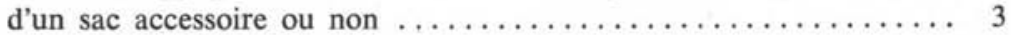

1. S.A. très hypertrophié, garni de 10 fortes épines de $20-25 \mu$. Cr. de $83 \mu$ H. (E.) paradoxus (Saakova) nov. comb. S.A. de taille plus proportionnée $(40 \mu)$, garni de nombreuses petites épines de $2,5 \mu . \mathrm{PC} / \mathrm{AM}=1 / 2$. Cirres glabres $\ldots \ldots \ldots \ldots \ldots \ldots 2$

2. Cr. de $65-72 \mu \ldots \ldots \ldots \ldots \ldots \ldots \ldots$. (E.) glareolae Singh. Cr. de $86-89 \mu$. . H. (E.) nitida Clerc $=$ H. (E.) dubininae nom. nov.

3. S.A. du type \& peigne atrial » (arc charnu garni de soies fines et longues), plus ou moins développé. $2 \times 4$ BMI $\ldots \ldots \ldots \ldots \ldots \ldots 4$

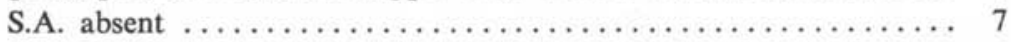

4. Vagin pourvu de sphincter et de fibres rétractrices. Cirres courts, glabres

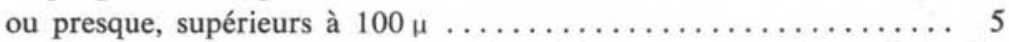
Vagin dépourvu de ces organes. Cirre épineux conique $(40 \times 7 \mu), \mathrm{Cr}$. de $87-92 \mu \ldots \ldots \ldots \ldots \ldots \ldots \ldots \ldots . \ldots \ldots$. (H.) crocethiae Webster.

5. S.A. très peu développé $(10 \times 5 \mu)$; soies de $13 \mu$. Vagin dépourvu de papilles. Cr. de $105-111 \mu \ldots \ldots \ldots \ldots \ldots$ H. (H.) etaplesensis $n . s p$. S.A. plus développés $(15$ ou $30 \mu)$. Vagins pourvus de papilles. Cirres

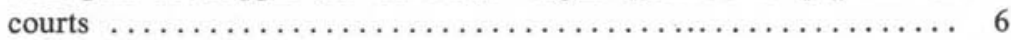

6. S.A. antérieur à l'atrium ; cirre glabre ; crochets de l'embryon de 18-20 $\mu$; Cr. du rostre de $108-110 \mu \ldots \ldots \ldots \ldots$ H. (H.) capetownensis $n$. sp. S.A. postérieur à l'atrium ; cirre ponctué ; crochets du rostre de $102 \mu$ $\ldots \ldots \ldots \ldots \ldots \ldots \ldots \ldots \ldots \ldots \ldots \ldots \ldots \ldots$. (H.) crocethia Belopolsk.

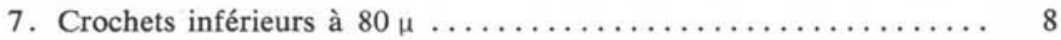

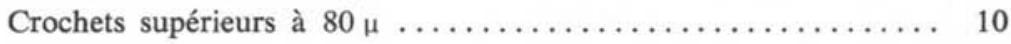

8. Crochets appartenant à trois espèces mal connues:

Crochets de $40-42 \mu$ H. (H.) paranitidulans (Golikowa) nov. comb. Crochets de $54-57 \mu \ldots \ldots \ldots \ldots \ldots$ H. nitidulans (Krabbe).

Crochets de $73-75 \mu \ldots \ldots \ldots \ldots$ H. (H.) magnisaccis Meggitt. Crochets appartenant à deux espèces mieux connues ; $2 \times 4$ BMI . . 9

9. Cr. de 57-64 $\mu$. Cirre invaginé court (25-30 $\mu$ ), armé ; vagin de 80-90 $\mu$ à dilatation chitinoïde ampullaire, dépourvu de sphincter et de fibres rétractrices $\ldots \ldots \ldots \ldots \ldots \ldots \ldots \ldots \ldots \ldots \ldots$. (H.) charadrii Yamag. Cr. de $68-71 \mu$. Cirre court $(25-30 \mu)$, glabre ; vagin de $40 \mu$ sans dilatation, mais pourvu d'un sphincter et de fibres rétractrices 


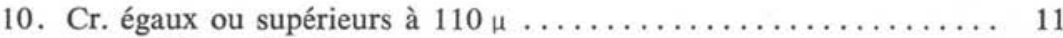

Cr. inférieurs à $100 \mu \ldots \ldots \ldots \ldots \ldots \ldots \ldots \ldots \ldots \ldots \ldots \ldots \ldots \ldots \ldots \ldots \ldots, 12$

11. P. du C. très courte : $\mathrm{PC} / \mathrm{AM}=1 / 10$. Test. en ligne ; ov. disposé entre les deux test. poraux. Cr. de $110-114 \mu \ldots \boldsymbol{H}$. (H.) moghensis Inamdar. P. du C. longue: $\mathrm{PC} / \mathrm{AM}=1 / 3$ à $1 / 2$. Vagin de $70 \mu$ à dilatation ampullaire de $7-15 \mu$. Cirre court très finement épineux .............

H. (H.) nitida (Krabbe).

12. $2 \times 4$ BMI. Strobile court $(3-4 \mathrm{~mm})$. Cr. de $94 \mu$. Cirre évaginé de 80-150 $\mu$, armé à la base. Vagin tubulaire très long $(170 \mu) \ldots \ldots \ldots \ldots$

H. (H.) lauriei Davies. Beaucoup plus de $2 \times 4$ BMI. Strobile de $40 \mathrm{~mm}$ au moins. Cr. de 84

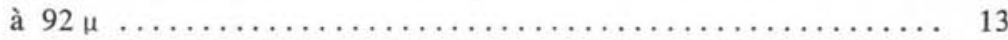

13. Cirres très puissants armés de fortes épines. Cr. voisins de $85-86 \mu 14$ Cirres longs et grêles armés de fines épines ou de soies $\ldots \ldots \ldots \ldots .15$

14. Cirre de $150 \times 20 \mu$ à fortes épines basales, suivies de soies. Vagin de plus de $200 \mu$, à papilles, coudé à $90^{\circ}$ par des fibres rétractrices ......

H. (H.) mudderbugtenensis (Baer).

Cirre de $90 \times 20 \mu$ à ornementation unique de fortes épines. Pas de soies. Vagin cylindrique sans papilles ni fibres rétractrices $\ldots \ldots \ldots \ldots \ldots \ldots$.

H. (H.) belopolskaiae n. sp.

15. Cirre long $(130 \mu)$, finement épineux sur $50 \mu$. Vagin tubulaire court $(80 \mu)$ et rectiligne. Cr. de $86-87 \mu \ldots \ldots \ldots \ldots \ldots$ H. (H.) litoralis Webster. Cirre de $80-90 \mu$, armé de soies sur $25 \mu$. Vagin conique de $55 \mu$, rectiligne. Cr. de $89-92 \mu \ldots \ldots \ldots \ldots \ldots \ldots$. . (H.) guschanskoi Krotov.

\section{Crochets claviformes}

Ventouses totalement inermes. Absence de sac accessoire ....... 1 Ventouses totalement ou partiellement armées (sur le bord ou sur le fond). Atrium pourvu d'un S.A. ou non ..................... 2

1. Présence de fines épines atriales. Crochets de $55 \mu$; P. du C. de $200 \mu$ environ. Cirre glabre de $54 \mu \ldots \ldots$ H. (H.) brachycephala (Creplin). Absence d'épines atriales .......................... 7

2. Ventouses d'Echinocotyle. Atrium armé pourvu d'un S.A. ....... 3 Ventouses d'Hymenolepis (Hym.). Atrium inerme dépourvu de S.A. Ovaire postérieur aux testicules; $\mathrm{P}$. du C. courte ; $\mathrm{PC} / \mathrm{AM}=1 / 6$ à

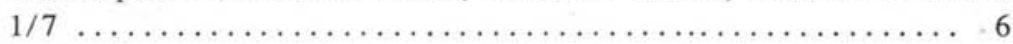

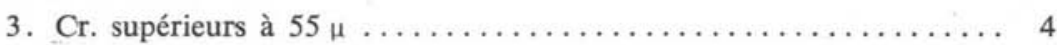

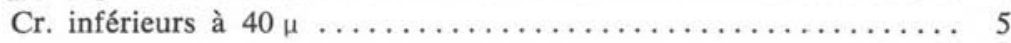

4. Cr. de 64-66 $\mu$. Test. en triangle (type D); P. du C. courte ; $2 \times 4$ BMI H. (E.) uralensis Clerc. 
Cr. de 58-62 $\mu$. Test. en ligne. Ovaire postérieur aux testicules et dépassant les $\mathrm{COR}$ à maturité. $\mathrm{PC} / \mathrm{AM}=1 / 3 \ldots$ H. (E.) hypoleuci Singh.

5. Cr. de 20-23 ; P. du C. courie: $\mathrm{PC} / \mathrm{AM}=1 / 7$. Cirre armé $\ldots \ldots \ldots$

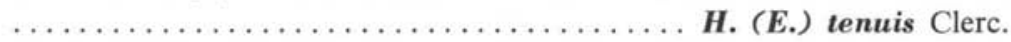

Cr. de $36-37 \mu ; \mathrm{P}$. du C. longue: $\mathrm{PC} / \mathrm{AM}=1 / 2$

H. (E.) longirostris (Rud.) sensu Baer, 1956.

6. Bord ventousaire armé. Cr. de $50-58 \mu \ldots \ldots$ H. (H.) oweni Moghe. Fond ventousaire armé. Cr. de $60-64 \mu$

H. (H.) quasioweni Dubinina.

7. a) Cirre inerme, court $(24 \mu)$; vagin tubulaire de $70 \mu$, pelotonné près de l'atrium. Cr. de $46-51 \mu \ldots \ldots \ldots \ldots$ H. (H.) cambrensis Davies.

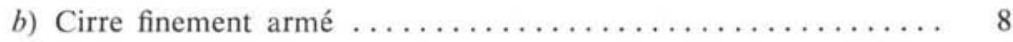

c) Cirres non connus de $s p$. inq. ................. 10

8. $2 \times 4$ BMI ; crochets élancés (à long manche) de $30 \mu$ environ .. 9 Plus de $2 \times 4 \mathrm{BMI}$; crochets de silhouette plus massive (à manche court), de $36 \mu$. Vagin conique, probablement chitinoïde, long de $100 \mu$ au moins. P. du C. $260 \mu . \mathrm{PC} / \mathrm{AM}=1 / 3 \ldots \ldots \ldots \ldots \ldots \ldots \ldots \ldots$

H. (H.) vaginata Baczinska.

9. Cr. de $30 \mu$. Cirre de $140 \mu$. P. du C. de 250-300 $\mu$ PC/AM $=1 / 3 \ldots$ H. (H.) styloides, Н. (H.) spinosa. Cr. de 27-32 $\mu$. Cirre de $200 \mu$ armé de soies. P. du C. de 350-400 $\mu$; $\mathrm{PC} / \mathrm{AM}=1 / 2$ à $1 / 3 \ldots \ldots \ldots \ldots$ H. (H.) clerci, H. (H.) fola.

10. Cr. de $27 \mu \ldots \ldots \ldots \ldots \ldots \ldots \ldots \ldots \ldots \ldots$. interrupta (Rud.).

Cr. de $40.43 \mu \ldots$ H. innominata Meggitt et H. rectacantha Fuhrm.

\section{F) CONCLUSION}

Les Charadrii du Nord de la France sont parasités par au moins neuf espèces différentes d'Hymenolepis (sensu lato). Une espèce est nouvelle : $H$. $(H$.$) etaplesensis; quatre étaient déjà connues de l'Ancien Monde : H .(H$. amphitricha (Rud.), $H$. (H.) charadrii Yamag., $H$. (H.) lauriei Davies et $H$. $(H$. nitida (Krabbe) ; une ne peut se différencier que par des caractères morphologiques mineurs de $H$. (H.) hughesi Webster décrite pour la première fois dans le Nouveau Monde; nous en avons fait une variété de cette dernière. Enfin, trois autres espèces ont été placées en species inquirendae, faute de scolex ou d'anneaux mûrs.

La détermination de ces Cestodes nous a conduits à entreprendre la revue bibliographique de la presque totalité des espèces décrites et à en critiquer certaines données contradictoires. Nous avons pu nous procurer des exemplaires d'une quinzaine d'espèces, dont parfois les types; quelques points 
d'anatomie mal connus ont alors été précisés, ou la description refaite entièrement. Il nous semble que parmi les 54 espèzes signalées à ce jour chez les Charadrii, la synonymie suivante doive être retenue : $H$. (H.) hughesi Webster, $1947=H$. $(H$.$) collariella Coil, 1956. Les synonymies entre H$. $(H$.) clerci$H$. (H.) fola d'une part et $H$. (H.) styloides-H. (H.) spinosa d'autre part peuvent être envisagées avec vraisemblance. Par contre, H. (H.) nitida (Krabbe, 1869) est bien différent de $H$. (E.) nitida Clerc, 1902-1903, Ces:ode pour lequel nous proposons le nouveau nom de Rymenolepis (Echinocotyle) dubininae (Clerc, 1902) nom. nov.

Si une trentaine d'espèces peuvent être considérées présentement comme bien connues ou, tout au moins, suffisamment connues, une quinzaine ont été placées en species inquirendae, soit que les données de la littérature les concernant soient trop sommaires et l'iconographie inexistante, soit qu'il nous ait semblé que plusieurs espèces différentes aient été décrites sous le même nom, soit enfin qu'il ne soit pas du tout certain qu'une espèce « retrouváe » soit effectivement semblable au type. L'exemple le plus caractéristique d'une telle confusion est représenté par le « comp!exe Hymenolepis recurvirostrae » dont nous avons amorcé le démembrement.

Ces Hymenolepis de Charadrii se révèlent difficilement classables dans d'autres genres que le genre Hymenolepis s.l., à l'exception des Cestodes du genre Echinocotyle dont les ventouses sont typiques et l'atrium génital armé, toujours pourvu d'un sac accessoire bien développé. Nous avons constaté par ailleurs la présence chez certains Hymenolepis (Hym.) d'un « sac » accessoire atrial d'un type particulier non sacculaire et pourvu de soies longues, que nous avons appelé « peigne atrial $\gg ;$. $(H$.$) crocethia, H .(H$.$) crocethiae,$ $H$. $(H$.$) capetownensis n. sp., H. (H.) etaplesensis n. sp. en sont pourvues.$

Il nous semble que les seuls caractères à retenir pour une détermination d'espèce soient les suivants, parce que remarquablement fixes: crochets du rostre, armature ventousaire, anatomie fine des conduits génitaux mâle et femelle (ces derniers malheureusement presque toujours ignorés), organes annexés à l'atrium (épines et sac accessoire des deux types), aspect et ornementation du cirre évaginé, muszulature interne du strobile, crochets de l'embryon, aspect des membranes de l'œuf mûr. Ces détails morphologiques sont beaucoup plus importants à noter et à décrire que ceux, néanmoins nécessaires, auxquels s'est attachée la tradition, parce que sujets à variation considérable suivant l'état de contraction, et de fraîcheur, du ver à la fixation : anatomie générale de l'anneau, avec les rapports de position des organes entre eux et, à un moindre degré, tailles du strobile, de l'anneau mûr et de la poche du cirre, lorsqu'elles sont correctement interprétées en fonction de l'état du ver. 


\section{Bibliographie}

Seules, les références postérieures à Fuhrmann, 1932 figurent dans notre liste.

Baer (J. G.), 1940. - Some avian tapeworm from Antigua. Parasit., 32, 174-197.

BAER (J. G.), 1956. - Parasitic helminths collected in West Greenland. Medd. om Gronland, 124, 55 p., Copenhague, Reitzels édit.

BAER (J. G.), 1959. - Helminthes parasites. Exploration des Parcs nationaux du Congo Belge, Mission J. G. Baer et W. Gerber (1958). Fasc. 1, 163 p. Inst. des Parcs nationaux du Congo Belge, Edit., (Tervuren, Belgique).

Belopolskaïa (M. M.), 1953. - Faune helminthologique des Chevaliers de I'U.R.S.S. Travaux d'Helminthologie en l'honneur du $75^{\circ}$ anniversaire de l'Acad. K. I. Skriabine, p. 47-65.

Belopol.skaïa (M. M.), 1954. - Faune parasitaire des oiseaux des réserves de Soudzhourinsk (Primoré). Uczenye Sapiski L.G.U., $\mathrm{n}^{\circ}$ 172, Sér. Biol., Vyp. 35.

Belopolskaïa (M. M.), 1959. - Faune parasitaire des Chevaliers de la Mer du Japon et de la Mer de Barentz. Ecologie Parasitaire, Léningrad, p. 22-57.

Conl (W. H.), 1956. - Two new hymenolepidid Cestodes from mexican birds, with observations on Hymenolepis crocethiae Webster, 1947. Jl. Parasitol., 42, 584-587.

DAviES (T. I.), 1939. - Four species of Hymenolepis Weinland parasitic in the oyster catcher Haematopus ostralegus Linn. Parasit., 31, 401-412.

Deblock (S.), Biguet (J.) et CAPron (A.), 1960. - Contribution à l'étude des Cestodes de Lari des côtes de France. I. Le genre Hymenolepis. Révision des espèces du genre à propos de trois descriptions dont une nouvelle. Ann. Par. Hum. et Comp., 35, 538-574.

Dubinina (M. N.), 1953. - Cestodes d'oiseaux nichant en Sibérie occidentale. Parasit. Sborn. Acad. Nauk. SSSR., 15, p. 117-232.

Fuhzmann (O.), 1932. - Les ténias des Oiseaux. Mém. de l'Université de Neuchâtel. VIII. Neuchâtel.

Golikowa (M. N.), 1959. - Recherches écologiques et parasitaires des biocénoses de quelques lacs de la région de Kaliningrad. Ecologie parasitaire, p. 150194.

Guinetsinskaïa (J. A.) et Naoumov (D. V.), 1958. - Faune helminthologique des Charadriiformes de la Mer Blanche. Trav. d'Helminthol. pour le $80^{\circ}$ ann. de l'académicien K:I. Skriabine, p. 99-108.

Hughes (R. C.), 1940. - The genus Hymenolepis Weinland, 1858. Oklahoma Agr. Exp. Stat. Techn. Bull., 8, 42 p.

INAMdaR (N. B.), 1934. - Four new species of avian Cestodes from India. Zeitschr. f. Parasitenk., 1935, 7, p. 198-206.

JoHRI (L. N.), - On a new avian Cestode belonging to the subfamily Hymenolepidinae Perrier, 1897 from Delhi State. Annual Number, 1960. Nat. Aca- 
demy of Sciences, India Allahabad 2, 1961, p. 70 (Abstract), in Biol. Record, 1962, $\mathrm{n}^{\circ} 7.563$.

JoyeuX (C.) et BAer (J.-G.), 1936. - Cestodes. Faune de France, t. 30, 613 p., Paris, Lechevalier édit.

Joyeux (C.) et BAER (J.-G.), 1939. - Sur quelques Cestodes de Madagascar. Bull. Soc. Path. Exot., 32, 39-43.

JoyeuX (C.) et BAer (J.-G.), 1940. - Sur quelques Cestodes. Rev. Suisse Zool., 47, p. 381-388.

Joyeux (C.), Baer (J.-G.) et Martin (R.), 1936. - Sur quelques Cestodes de la Somalie Nord. Bull. Soc. Path. Exot., 29, p. 82-85.

Krotov (A. I.), 1952. - Nouveaux Cestodes (Hymenolepididae et Paruterinidae) d'oiseaux. Trud. Gelmint. Lab. Akad. Nauk. SSSR., 6, 259-272.

López-Neyra (C.), 1942. - Division del genero Hymenolepis Weinland (s.l.) en otros mas naturales. Rev. Iber. Parasit., 2, p. 46-93 et 113-256.

MegGitT (F. J.), 1927. - Report on a collection of Cestoda mainly from Egypt. Part II. Cyclophyllidea: Family Hymenolepididae. Parasitol., 19, p. 420450.

MeggrtT (F. J.), 1933. - Cestodes collected from animals dying in the Calcutta Zoological gardens during 1931. Rec. Ind. Mus., 35, p. 145-165.

Moghe (M. A.), 1933. - Four new species of avian Ces:odes from India. Parasitol., 25 , p. 333-341.

PAnine (V.-Y.), 1960. - Faune helminthologique des oiseaux de la vallée du Zaysan. Troudi Inst. Zool. Acad. Nauk. Kazak. SSSR, 12, p. 166-172.

RYBicka (K.), 1958. - Cestodes d'Oiseaux (sauf Ansériformes) du Lac Druzno (Parasitofaune de la biocénose du Lac Druzno, part IV). Acta Par. Pol., 6, p. 143-178, 11 fig.

SaAkova (Z. O.), 1958. - Deux nouveaux genres de Cestodes de la famille des Hymenolepididae chez des oiseaux du delta du Danube. Travaux d'Helminthologie pour le $80^{\circ}$ anniversaire de l'académicien K.I. Skriabine, p. $310-312$, fig. 1-4.

Sandeman (I. M.), 1959. - A contribution to the revision of the dilepid tapeworms from Charadriiformes. Preliminary note. Zoolog. Anzeig., 163 (9,10), 278-288.

SHen Tseng, 1933. - Studies on avian Cestodes from China. Part II. Cestodes from charadriiform birds. Parasitology, 1933, 24, p. 500-511.

SINGH (K.), 1952. - Cestodes parasites of birds. Indian Jl. Helminth., 4, p. 1-72.

Skriabine (K. I.) et Mathevossian (E. M.), 1942. - Corrections d'erreurs et de contradictions de la taxonomie des Cestodes de la famille des Hyménolépididés. Dokl. Acad. Nauk. SSSR, 3ó (6), p. 205-207, in Spassky-Spasskaïa, 1954.

Skriabine (K. I.) et Mathevossian (E. M.), 1945. - Helminthes rubannés - Hyménolépididés - des Oiseaux domestiques et sauvages. Moscou, 486 p., in Spassky-Spasskaïa, 1954. 
SPASSKY (A. A.), 1959. - De l'origine polyphylétique des Hymenolepididae du genre Oligorchis Fuhrmann. Trudi Gelm. Lab. Acad. Nauk. SSSR, 9, p. 296-310.

Spassky (A. A.) et Bobova (L. P.), 1962. - Cestodes de la famille des Hymenolepididae des oiseaux aquatiques du Kamtchatka. Trudi Gelm. Labor. Acad. Nauk. SSSR, 1962, 12, p. 172-199.

Spassky (A. A.) et Spasskaïa (L. P.), 1954. - Construction d'une systématique des Hyménolépididés parasites d'oiseaux. Trud. Gelm. Lab. Akad. Nauk. SSSR, 1, p. 55-119.

Voge (M.) et ReAd (C. P.), 1954. - A description of Parafimbriaria websteri n. g., n. sp., a Cestode from grebes, and notes on three species of Hymenolepis. Jl. Parasit., 1954, 40 (4), p. 564-570.

Webster (J. D.), 1947. - Studies on the genus Hymenolepis with description of three new species. Jl. Parasit., 33, 99-106.

Yamaguti (S.), 1935. - Studies on the helminth fauna of Japan. Part 6. Cestodes of birds I. Japan Jl. Zool., 6, 183-232.

YAMAGUTI (S.), 1940. - Studies on the helminth fauna of Japan. Part 30. Cestodes of birds II. Japan Jl. Med. Sc., sect. VI, vol. I, p. 175-211.

YAMAGUtI (S.), 1959. - Systema helminthum, vol. II. The Cestodes of vertebrates, 626 p., 70 planches, Interscience Publishers, New-York et Londres.

(Travail du Laboratoire de Parasitologie de la Faculté Mixte de Médecine et Pharmacie de Lille; Directeur: Professeur J. BIGUET) 